\title{
14. NEOGENE TEPHROCHRONOLOGY FROM SITE 758 ON NORTHERN NINETYEAST RIDGE: INDONESIAN ARC VOLCANISM OF THE PAST 5 MA $^{1}$
}

\author{
J. Dehn, ${ }^{2}$ J. W. Farrell, ${ }^{3}$ and H.-U. Schmincke ${ }^{2}$
}

\begin{abstract}
A tephrochronology of the past $5 \mathrm{Ma}$ is constructed with ash layers recovered from Neogene sediments during drilling at ODP Leg 121 Site 758 on northern Ninetyeast Ridge. The several hundred tephra layers observed in the first $80 \mathrm{~m}$ of cores range in thickness from a few millimeters to $34 \mathrm{~cm}$. Seventeen tephra layers, at least $1 \mathrm{~cm}$ thick, were sampled and analyzed for major elements. Relative ages for the ash layers are estimated from the paleomagnetic and $\delta^{18} \mathrm{O}$ chronostratigraphy.

The ash layers comprise about $1.7 \%$ by volume of the sediments recovered in the first $72 \mathrm{~m}$. The median grain size of the ashes is about $75 \mu \mathrm{m}$, with a maximum of $150 \mu \mathrm{m}$. The ash consists of rhyolitic bubble junction and pumice glass shards. Blocky and platy shards are in even proportion $(10 \%-30 \%)$ and are dominated by bubble wall shards $(70 \%-90 \%)$. The crystal content of the layers is always less than $2 \%$, with plagioclase and alkali feldspar present in nearly every layer. Biotite was observed only in the thickest layers.

The major element compositions of glass and feldspar reflect fractionation trends. Three groupings of ash layers suggest different provenances with distinct magmatic systems. Dating by $\delta^{18} \mathrm{O}$ and paleomagnetic reversals suggests major marine ash-layer-producing eruptions (marine tephra layers $>1 \mathrm{~cm}$ in thickness) occur roughly every approximately $414,000 \mathrm{yr}$. This value correlates well with landbased studies and dates of Pleistocene Sumatran tuffs (average 375,000-yr eruptive interval). Residence times of the magmatic systems defined by geochemical trends are $1.583,2.524$, and $1.399 \mathrm{Ma}$. The longest time interval starts with the least differentiated magma.

The Sunda Arc, specifically Sumatra, is inferred to be the source region for the ashes. Four of the youngest five ash layers recovered correlate in time and in major element chemistry to ashes observed on land at the Toba caldera.
\end{abstract}

\section{INTRODUCTION}

\section{Purpose of this Study}

The tephra layers recovered in the Pliocene to Holocene sediments of Ocean Drilling Program (ODP) Leg 121 Site 758 (Fig. 1) provide a unique record of explosive volcanism in the northeastern Indian Ocean. Several hundred discrete ash layers, ranging from a few millimeters to $34 \mathrm{~cm}$ in thickness, were observed in Holes 758A, 758B, and 758C drilled on northern Ninetyeast Ridge (Peirce, Weissel, et al., 1989). The excellent core recovery through the Pliocene $(102 \%)$, large volume of ash $(1.7 \%$ bulk sediment), and freshness of the glass are ideal for a tephrochronological study. This study focuses on the youngest of these tephra layers $(0.075$ to $5.1 \mathrm{Ma})$.

Case studies in tephrochronology are especially useful for determining the (1) source region of the ashes; (2) volcanic, magmatic, and temporal evolution of the source regions; (3) eruptive cycles; and (4) lithostratigraphic and chronostratigraphic correlations in marine sedimentary sequences when numerically dated (Bitschene and Schmincke, 1990). The aim of this case study is to develop a tephrochronology for Site 758 using the stratigraphy, ages, and chemical compositions of the major ash layers. Analyses were performed on 29 samples taken from 17 major ash layers, labeled $\mathrm{A}$ through $\mathrm{M}$, which had a thickness of $1 \mathrm{~cm}$ or more (Fig. 2).

\footnotetext{
${ }^{1}$ Weissel, J., Peirce, J., Taylor, E., Alt, J., et al., 1991. Proc. ODP, Sci. Results, 121: College Station, TX (Ocean Drilling Program).

2 GEOMAR, Forschungszentrum für Marine Geowissenschaften der ChristianAlbrechts-Universităt zu Kiel, Wischhofstraße Gebăude 3, D-2300 Kiel 14, Federal Republic of Germany.

${ }^{3}$ Department of Geology, Brown University, Providence, RI 02912-1846, U.S.A.
}

\section{Regional Setting of Site 758}

Site 758 is at $5^{\circ} 23.05^{\prime} \mathrm{N}, 90^{\circ} 21.67^{\prime} \mathrm{E}$, on the crest of Ninetyeast Ridge between Deep Sea Drilling Project (DSDP) Sites 216 and 217 (Fig. 1). The water depth at Site 758 is $2924 \mathrm{~m}$. At this locality, Ninetyeast Ridge comprises several en echelon blocks. Site 758 , which is on the southeast side of one such block, lies at least $1000 \mathrm{~m}$ above the Bengal Fan.

Site 758 is approximately $1000 \mathrm{~km}$ from the nearest volcanically active region, Sumatra. Sumatra is the northernmost part of the Sunda Arc and has at least one restive caldera. The most recent eruption in this region was from the Toba caldera at approximately 75,000 yr (Ninkovich et al., 1979; Rose and Chesner, 1987, 1988). This caldera, one of the largest Quaternary calderas on Earth (Smith and Bailey, 1968), is probably the source for several of the tephra layers.

\section{Previous Studies of Northeastern Indian Ocean Tephras}

Most previous work has been performed on the youngest eruption of the Toba Caldera (Ninkovich et al., 1979; Ninkovich, 1979; Rose and Chesner, 1987; Chesner, 1988). These studies are primarily land based, though some authors have studied piston cores containing marine tephra layers correlated to the eruptions (Ninkovich et al., 1979; Ninkovich, 1979; Kennett, 1981). The Toba ash, as it is called in the literature, has been discovered at many localities in the northeastern Indian Ocean (Ninkovich et al., 1979; Fig. 1). At least $800 \mathrm{~km}^{3}$ of ash was erupted from Toba (Rose and Chesner, 1987) and fell over a minimum area of $5 \times$ $10^{6} \mathrm{~km}^{2}$, mostly over the Bay of Bengal (Ninkovich et al., 1979; Fig. 1). The distribution of the ash may have reached as far as $3000 \mathrm{~km}$ (Williams and Royce, 1982). Because Site 758 lies within this area, the recovery of the Toba ash and other tephra layers was expected.

Older eruptions of the Toba caldera complex occurred at 0.84 (Diehl et al., 1987) and 1.20 Ma (Nishimura et al., 1977). Chesner 


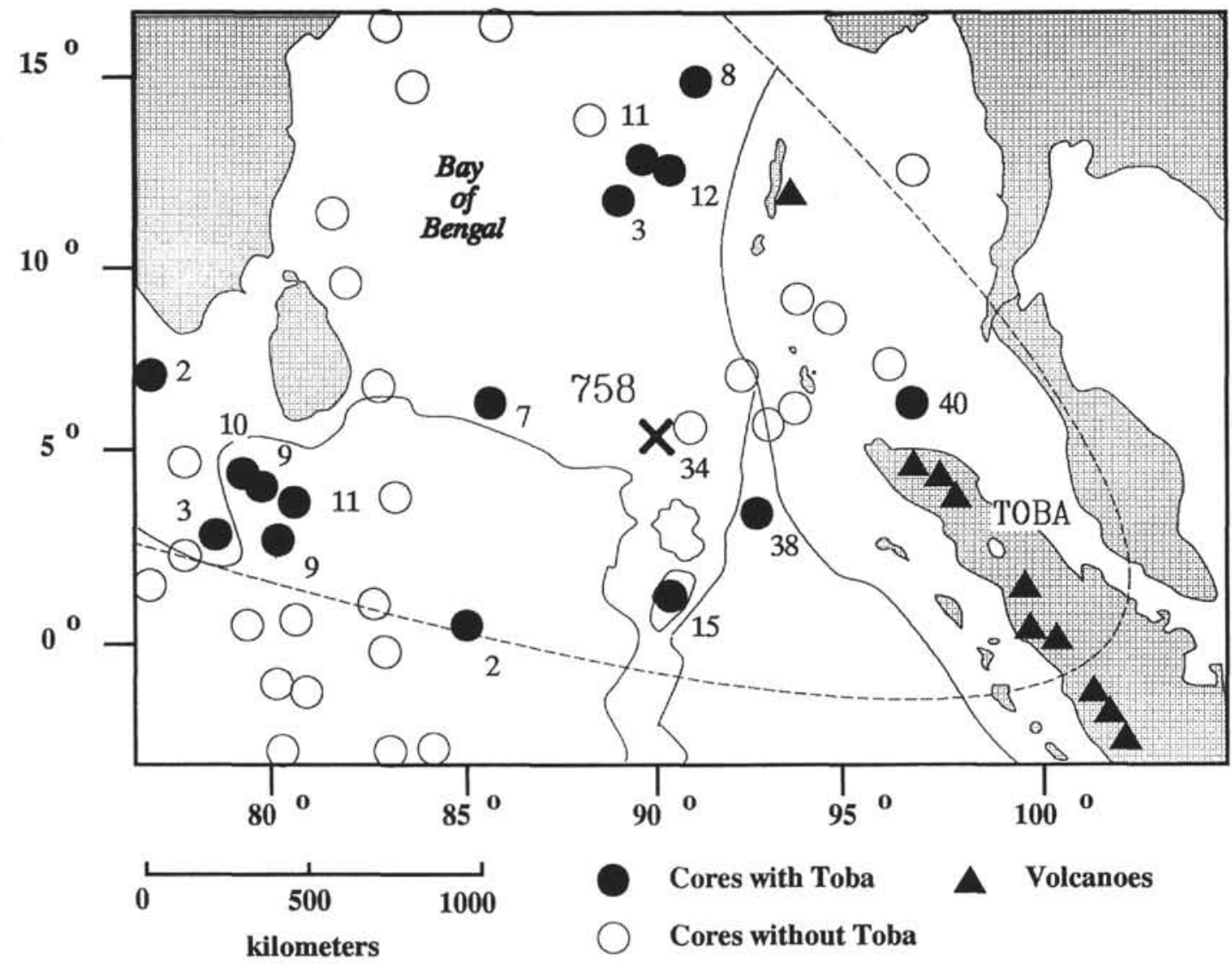

Figure 1. Site 758 and other locations in the northeastern Indian Ocean where the youngest tephra layer from the Toba caldera $(75,000 \mathrm{yr})$ was recovered. The thickness of the layers in centimeters accompanies each point (after Ninkovich et al., 1979; Ninkovich, 1979).

(1988) studied the last four eruptions of the Toba caldera at 0.075 , $0.450,0.840$, and $1.2 \mathrm{Ma}$. He described these layers as the youngest Toba tuff (YTT), the middle Toba tuff (MTT), the oldest Toba tuff (OTT), and the Haranggoal dacite tuff (HDT). These tuffs are tentatively correlated to the Site 758 tephra layers as follows: YTT to layer A, MTT to layer C, OTT to layer E, and HDT to layer F. (A detailed description of the nomenclature follows.)

The drill cores previously recovered in the northeastern Indian Ocean have not been suitable for the reconstruction of a continuous tephrochronology. The sedimentary records of Sites 216 and 217 are marred by poor recovery and disturbance from rotary drilling (von der Borch, Sclater, et al., 1974). Piston cores from the Bay of Bengal, such as those recovered aboard the Vema and the Conrad, provide continuous and undisturbed records, but sample only the near-surface Pleistocene sediments (Ninkovich, 1979).

\section{METHODS}

Based upon shipboard observation, 29 samples representing 17 discrete ash layers were taken (Table 1). The grain size, morphology, and crystal components of the ashes were studied in smear slides and thin sections. Between 15 and 20 shards were measured in each slide by calibrated ocular scales.

The major element compositions of the glass shards and mineral grains were determined using an ARL electron microprobe with natural glass and mineral standards at the Department of Mineral Sciences, Smithsonian Institution. Operating conditions were $15-\mathrm{kV}$ accelerating voltage, $10-\mu \mathrm{m}$ beam spot, and 14-nA beam current. Measured $\mathrm{Na}_{2} \mathrm{O}$ and $\mathrm{K}_{2} \mathrm{O}$ concentrations may be slightly lower (up to $1 \%$ ) as a result of selective sodium and potassium loss during the microprobe analysis.

\section{DESCRIPTION OF THE TEPHRA LAYERS}

\section{Stratigraphy and Lithology}

The ash layers were recovered in the nannofossil ooze of Unit I at Site 758 (Peirce, Weissel, et al., 1989). This unit consists of two subunits: IA is a nannofossil ooze with foraminifers and clay and clayey nannofossil ooze with foraminifers from 0 to $25.2 \mathrm{~m}$ below seafloor (mbsf), and IB is a nannofossil ooze with clay, foraminifers, and micrite from 25.2 to $121.7 \mathrm{mbsf}$. The ash layers are present in Pliocene (5.1 Ma) and younger sediments.

Many of the tephra layers are present in one hole, but not in the neighboring one. All but one of the layers fall into the intervals between cores, where as much as $2.7 \mathrm{~m}$ of sediments is not recovered. This "under recovery" (Ruddiman et al., 1987) is a product of the coring method and is surmounted by constructing a composite stratigraphy. A detailed composite stratigraphy for this section is presented by Farrell and Janecek (this volume). The remaining tephra layer does not appear to correlate among the holes. The unique layer B in Hole $758 \mathrm{~A}$ could be the result of reworking of the sediments by slumping, bottom currents, or bioturbation (Bramlette and Bradley, 1942; Ruddimann and Glover, 1982).

\section{Macroscopic Description of the Ashes}

Tephra layers with thicknesses of $1 \mathrm{~cm}$ or more are defined as "major." Seventeen such layers were identified in the first $5.1 \mathrm{Ma}$ (0-72 mbsf) of Site 758 and labeled A through M, with A the 


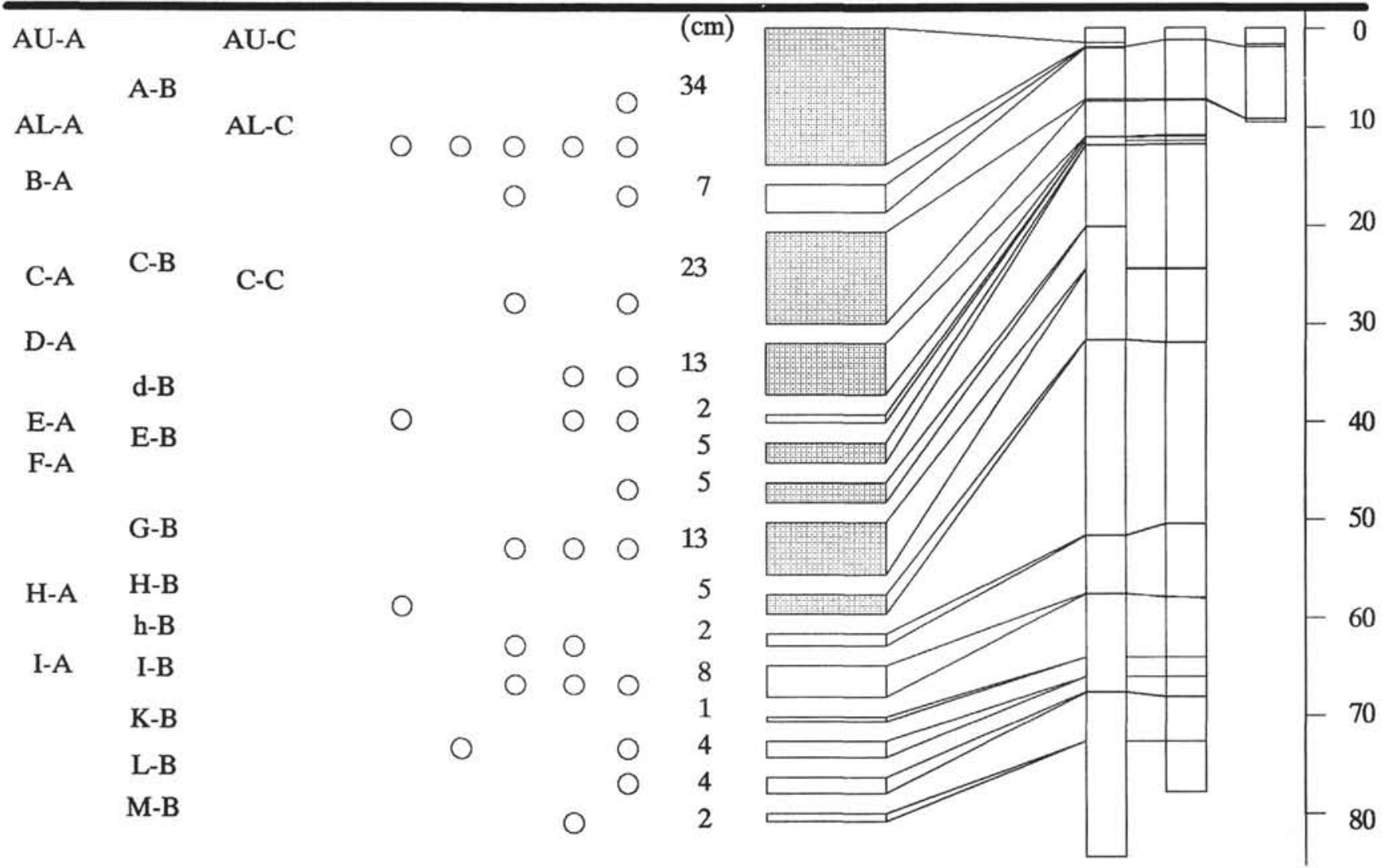

Figure 2. Tephrostratigraphic diagram of the upper Neogene tephras at Site 758. The samples are coded by layer $(\mathrm{A}-\mathrm{M})$, stratigraphic indicator $(\mathrm{U}=$ upper, $\mathrm{L}=$ lower), and hole (A, B, or C). The minerals phases measured in each sample are represented by the circles. Quartz was present in every layer sampled; the other mineral phases measured in the samples are APAT $=$ apatite, $\mathrm{AMPH}=$ amphibolite, $\mathrm{BIOT}=$ biotite, $\mathrm{SAN}=$ sanidine, and PLAG $=$ plagioclase. The thickness of the layers is shown by the relative scale of the boxes; shading indicates layers dated by $\delta^{18} \mathrm{O}$. Note that layer B is not present in Holes $758 \mathrm{~B}$ and $758 \mathrm{C}$.

Table 1. Major ash layers and their maximum age range defined by paleomagnetic and $\delta^{18} \mathrm{O}$ dating, thickness, depth, and sample locations at Site 758.

\begin{tabular}{|c|c|c|c|c|c|c|}
\hline \multirow[b]{2}{*}{ Layer } & \multirow{2}{*}{$\begin{array}{c}\text { Maximum } \\
\text { age range } \\
\text { (Ma) }\end{array}$} & \multirow{2}{*}{$\begin{array}{l}\text { Thickness } \\
(\mathrm{cm})\end{array}$} & \multirow{2}{*}{$\begin{array}{l}\text { Depth } \\
\text { (mbsf) }\end{array}$} & \multicolumn{3}{|c|}{ Core, section, interval $(\mathrm{cm})^{\mathrm{a}}$} \\
\hline & & & & $758 \mathrm{~A}$ & $758 \mathrm{~B}$ & $758 \mathrm{C}$ \\
\hline A (upper) & $0.071-0.110$ & 34 & $1.50-1.84$ & $1 \mathrm{H}-2,5$ & & $1 \mathrm{H}-2,14$ \\
\hline$A$ (mixed) & $0.071-0.110$ & 34 & $1.50-1.84$ & & $1 \mathrm{H}-1,114$ & \\
\hline A (lower) & $0.071-0.110$ & 34 & $1.50-1.84$ & $1 \mathrm{H}-2,25$ & & $1 \mathrm{H}-2,27$ \\
\hline $\mathrm{B}(\mathrm{A}$ ?) & $0.071-0.121$ & 7 & $1.94-2.01$ & $1 \mathrm{H}-2,47$ & & \\
\hline C & $0.512-0.538$ & 23 & $7.12-7.35$ & $2 \mathrm{H}-1,114$ & 1H-5, 119 & $1 \mathrm{H}-7,1$ \\
\hline D & $0.731-0.750$ & 13 & $10.80-10.93$ & $2 \mathrm{H}-4,38$ & & \\
\hline d & $0.756-0.760$ & 2 & $11.25-11.27$ & & $2 \mathrm{H}-2,25$ & \\
\hline E & $0.774-0.780$ & 5 & $11.62-11.67$ & $2 \mathrm{H}-4,115$ & $2 \mathrm{H}-2,61$ & \\
\hline F & $1.273-1.294$ & 5 & $19.82-19.87$ & $3 \mathrm{H}-3,125$ & DNE & \\
\hline G & $1.596-1.653$ & 13 & $24.00-24.13$ & DNE & $3 \mathrm{H}-4,69$ & \\
\hline H & $2.238-2.265$ & 5 & $31.20-31.25$ & $4 \mathrm{H}-5,3$ & $4 \mathrm{H}-3,8$ & \\
\hline $\mathrm{h}$ & $3.664-3.666$ & 2 & $49.67-49.70$ & & $6 \mathrm{H}-2,47$ & \\
\hline I & $4.124-4.131$ & 8 & $56.67-56.75$ & $7 \mathrm{H}-2,120$ & $6 \mathrm{H}-7,17$ & \\
\hline J & $4.552-4.560$ & 1 & $63.12-63.13$ & DNE & & \\
\hline $\mathrm{K}$ & $4.666-4.667$ & 4 & $65.09-65.13$ & & $7 \mathrm{H}-6,29$ & \\
\hline L & $4.759-4.765$ & 4 & $66.66-67.00$ & & 7H-CC, 14 & \\
\hline \multirow[t]{4}{*}{ M } & $5.062-5.063$ & 2 & $71.61-71.63$ & & $8 \mathrm{H}-4,111$ & \\
\hline & & & & $1 \mathrm{H}-11 \mathrm{H}$ & $1 \mathrm{H}-10 \mathrm{H}$ & $1 \mathrm{H}$ \\
\hline & & & & $12 \mathrm{X}-46 \mathrm{X}$ & & \\
\hline & & & & $47 R-73 R$ & & \\
\hline
\end{tabular}

a Tephra layers lost between cores are marked "do not exist" (DNE). 
youngest layer and $\mathrm{M}$ the oldest. Layers identified by peaks in the magnetic susceptibility of the sediments (up to $113 \times 10^{-6} \mathrm{cgs}$ ) are denoted by capital letters. The lowercase letters ( $\mathrm{d}$ and $\mathrm{h}$ ) identify those ash layers visible in the cores, but not readily identified by large susceptibility peaks. Because these two layers are thin $(2 \mathrm{~cm})$ in comparison with the other layers, it is probable that they fall between points of the susceptibility measurement.

The major tephra layers appear as gray to grayish brown layers of varying thickness (Fig. 2). All the ash layers have sharp basal contacts to the host sediment. The upper contacts are gradational and show normal grading in size and density as well as tephra concentration in the host sediment. The ashes are primarily composed of glass shards, pumice fragments, and crystals. No lithic clasts were observed in any of the layers.

The tephra layers are marine fallout ash layers formed by explosive eruptions that dispersed the tephra, allowing it to accumulate over a large area (to a distance of at least $2500 \mathrm{~km}$ ). The tephra was deposited on the ocean surface and then settled through more than $2000 \mathrm{~m}$ of water. After deposition, reworking by bottom currents and bioturbation of the ash layers diffused the tephra within the host sediment. The combination of these fragmentation, transport, and reworking mechanisms leads to the observed grading of the tephra. All of the layers exhibit weak normal grading of particle density, grain size, and particle concentration in the host sediment (Fig. 3). Crystals are rare, comprising less than $1 \%$ of the ash volume with the exception of layers $\mathrm{B}$ and d, each of which has approximately $2 \%$ crystals by volume. Crystals, where present, are concentrated at the base of a layer.

The thicknesses of the ash layers are based on visual inspection, core photograph interpretation, and magnetic susceptibility. The thicknesses of the major tephra layers vary widely to a maximum of $34 \mathrm{~cm}$ for layer $\mathrm{A}$ and with an average thickness of $9 \mathrm{~cm}$ (Table 1). Individually the layers appear to be a minor component, yet together they comprise up to $1.7 \%$ of the sediments recovered through the Pliocene. Thickness is the most obvious difference between the tephra layers (Fig. 2). The thickest layers, G, D, C, and A, represent two-thirds of the ash by volume. The remaining layers, B, d, E, F, H, h, I, J, K, L, and M, have an average thickness of only $4 \mathrm{~cm}$.

Most of the layers correlate among the holes at Site 758, and those that are not present in a hole usually fall into an interval lost between cores. Layer B in Hole 758 A, however, does not correlate to any tephra layer in Holes $758 \mathrm{~B}$ and $758 \mathrm{C}$. Furthermore, this layer falls into an interval fully recovered in all three holes at Site 758. This tephra layer is atypical in other aspects as well. Layer $\mathrm{B}$ is crystal rich $(2 \%)$ and has a large grain size (1-2 mm) relative to the other ashes. It occurs only $12 \mathrm{~cm}$ below layer $A$ and has an angular contact to the surrounding sediments (Fig. 3). Both the upper and lower contacts to the nannofossil ooze are sharp. Because it is so different from the other tephra layers and because it lies so close to layer A, this layer is thought to be a basal part of layer A, an ash pod, displaced downward in the core by slumping or reworking by bottom currents. The size and thickness of the pod $(7 \mathrm{~cm})$ seem to rule out bioturbation. The grain size and high crystal concentration support the idea of a slump of denser material in the surrounding ooze. There is no sign of such disturbance below layer $\mathrm{A}$ in Cores $121-758 \mathrm{~B}-1 \mathrm{H}$ or $121-758 \mathrm{C}-1 \mathrm{H}$, which are in holes $100 \mathrm{~m}$ away. The latter core was severely disturbed by drilling and evidence of such a slump could be obscured.

Layer D was also not observed in Hole $758 \mathrm{~B}$, though this interval was recovered. Visual inspection of the cores shows an ash layer, d, at the stratigraphic location where D would be expected. Layer $\mathrm{d}$ is possibly a diffused or reworked tephra D.

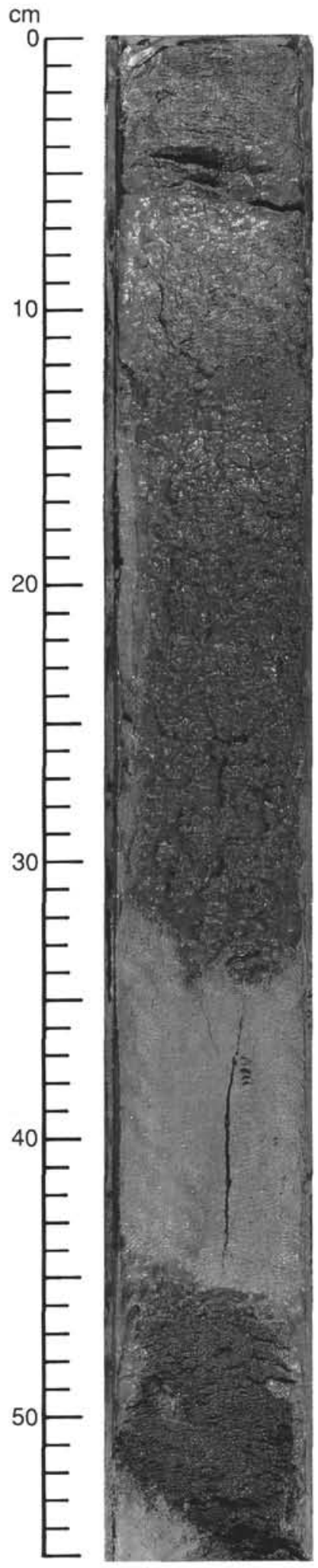

Figure 3. Core photograph of the Toba ash (layers A and B). Layer A has an expanded, yet typical, morphology of the tephra layers, with a sharp basal contact to the host sediment and a gradational upper contact. Where present, crystals are concentrated at the base of each tephra layer, indicating a density gradation. Layer B may be reworked from layer A. 
Further evidence of the relationship between layers A and B and layers $\mathrm{D}$ and $\mathrm{d}$ based on their chemical compositions is discussed in greater detail below.

\section{Microscopic Description of the Ashes}

The ash layers are a concentrated, yet loose, aggregation of glass shards, pumice, and crystals within organic sediments. The glass shards have a median grain size of $75 \pm 25 \mu \mathrm{m}$, with a maximum size of $150 \mu \mathrm{m}$. Bubble wall shard forms dominate $(70 \%-90 \%)$, followed by platy and blocky shards $(10 \%-30 \%$ each) and crystals (typically $<1 \%$ ). The refractive index of the glass is low (less than 1.54) indicating a silica content of $70 \%$ or greater (Schmincke, 1981). No brown volcanic glass of basaltic composition was observed in any sample. The glass is always isotropic and does not appear altered. Fractures, hydration cracks, or incrustation were not observed in smear slides or thin sections. The glass is probably slightly hydrated through interaction with seawater and/or interstitial water, but significant alteration has not yet taken place.

Quartz and feldspars comprise the bulk of the crystals in the tephra layers, followed by biotite, apatite, and amphibole. Quartz is present in every tephra layer sampled. Plagioclase is common in $30-60-\mu \mathrm{m}$ subhedral crystals displaying twinning but little or no zoning. Alkali feldspars occur in layers A, D, d, G, h, I, and M as larger, $40-80 \mu \mathrm{m}$, euhedral to subhedral crystals. Only the crystal-rich layers B, d, G, and I contain iron-bearing minerals, chiefly biotite and minor amphibole.

The grain sizes of the tephra $(50-150 \mu \mathrm{m})$ suggest that the transport distance was approximately $1000 \mathrm{~km}$ (Fisher, 1964; Walker, 1971). This supports the conclusion that the tephras came from nearest volcanically active region, Sumatra.

\section{GEOCHEMISTRY OF THE TEPHRA LAYERS}

Major element concentrations were determined for single glass shards and mineral grains using an electron microprobe. Volcanic glass of different morphology was analyzed in every sample, and plagioclase and potassium feldspars were analyzed wherever present, as well as other crystal phases.

\section{Geochemistry of the Glass Shards}

The analyzed volcanic glasses are isotropic and appear fresh with major oxide sums ranging between $90 \%$ and $97 \%$. Three general morphological categories of shards were chosen for analysis: platy, bubble wall, and blocky. These three forms were measured in each sample, but no significant change in major oxide composition was seen as a function of shard morphology. An average of 10 shards was analyzed per thin section, with a minimum of one thin section per sampled layer (Table 2). Layer J was not sampled for chemical analysis because it was identified as a "major" layer only after onshore analysis of shipboard records. All the glasses are classified as high-silica rhyolites with $\mathrm{SiO}_{2}$ concentrations of $72 \%$ to nearly $79 \%$ and $\mathrm{MgO}$ concentrations of less than $1 \%$.

Plots of major oxide sums subtracted from 100 give rough estimates of the percentage of water in the glasses. The inferred water contents are a result of hydration during subaqueous alteration and not of the original volatile content in the magma. The inferred water contents show a slight drop from $6 \%$ to $5 \%$ at 35 mbsf. The break in the water contents roughly corresponds with the break between Subunits IA and IB (Pierce, Weissel, et al., 1989) and thus the clay and carbonate content in the surrounding sediments. This relation suggests that the preservation of the glasses, and thus the change in the water content, is partially influenced by the host sediment and interstitial water.
Variation diagrams of $\mathrm{FeO}, \mathrm{CaO}, \mathrm{Al}_{2} \mathrm{O}_{3}$, and $\mathrm{K}_{2} \mathrm{O}$ vs. $\mathrm{SiO}_{2}$ (Fig. 4) all show a distinct grouping by ash layer. Layer L stands out in every plot as the least evolved, with concentrations of $2.43 \%-2.65 \% \mathrm{FeO}$ and $72.25 \%-73.28 \% \mathrm{SiO}_{2}$. Layer $\mathrm{A}$, inferred to be the Toba ash from the 75,000-yr eruption of the Toba caldera in Sumatra, is the most evolved layer, with concentrations of $76.65 \%-78.89 \% \mathrm{SiO}_{2}$ and $0.72 \%-1.03 \% \mathrm{FeO}$. Layers $\mathrm{A}$ and $\mathrm{B}$ have similar compositions of major oxides, supporting the conclusion that layer B is a displaced pod of layer A. Layers D and d are also chemically indistinguishable. Coupled with their stratigraphic correlation, the chemistry of these two layers suggests that they could be the same layer in each hole.

Chemical trends are visible for $\mathrm{CaO}, \mathrm{FeO}$, and $\mathrm{Al}_{2} \mathrm{O}_{3}$ vs. $\mathrm{SiO}_{2}$. Increasing silica concentrations correlate with lower $\mathrm{CaO}, \mathrm{FeO}$, and $\mathrm{Al}_{2} \mathrm{O}_{3}$ concentrations. These trends are expected, but do not correlate directly with depth, and thus the age, of the tephra layer. Layers L and E, although significantly different from the other layers in composition, lie along the trends formed by the other tephras. $\mathrm{FeO}$ content increases slightly with very high $\mathrm{SiO}_{2}$ contents in analyses from layers $\mathrm{H}$ and $\mathrm{A}$ (increasing from $0.6 \%$ to $0.8 \% \mathrm{FeO}$ with $\mathrm{SiO}_{2}$ of $78 \%-79 \%$ ). The $\mathrm{K}_{2} \mathrm{O}$ vs. $\mathrm{SiO}_{2}$ diagram shows groupings by tephra layer, but no clear trend. A trend in this data could be obscured as a result of the large scatter in values of $\mathrm{K}_{2} \mathrm{O}$. This scatter is probably caused by inaccuracies in measurement due to sodium and potassium evaporation during the microprobe analysis or a gain in potassium through low-temperature alteration.

Though no empirical correlation between age and chemical composition is seen, several of the ashes are clearly related. A diagram of $\mathrm{CaO}$ vs. FeO (Fig. 5) displays three trends, one which links layers A, B, C, D, d, E, and F (Group 1), one relating H, K, and $\mathrm{L}$ (Group 2), and one relating I and $\mathrm{M}$ (Group 3 ). These same groupings are less clear, though present, for $\mathrm{Al}_{2} \mathrm{O}_{3}$ vs. $\mathrm{FeO} . \mathrm{CaO}$ vs. $\mathrm{Al}_{2} \mathrm{O}_{3}$ shows an inverted, yet similar, relationship between these layers. These groupings of layers, though different in their element concentrations, all have similar element ratios. Since $\mathrm{Zr}$ and $\mathrm{Nb}$ cannot be measured by microprobe, $\mathrm{Ca}$ and $\mathrm{Fe}$ are used to determine cogenetic trends in the ash layers. The correlation of these ash layer groupings could be supported by similar trends in $\mathrm{MgO}$ vs. $\mathrm{FeO}$, but the $\mathrm{MgO}$ concentrations are too small to clearly distinguish the relationships of the tephra layers.

In order to examine the change in the major oxides with age, plots were made of major oxides and major oxide ratios vs. depth (Fig. 6). Depth is plotted rather than age because different methods were used to date the tephras and the measured ages vary by up to $0.04 \mathrm{Ma}$ between methods. A more detailed discussion of the age of the tephra layers follows.

Certain oxides, such as $\mathrm{Al}_{2} \mathrm{O}_{3}$, show considerable scatter within a single tephra layer. Others, such as $\mathrm{FeO}, \mathrm{CaO}$, and $\mathrm{SiO}_{2}$, are very well restrained within each layer. No general trend is visible for the entire suite of layers; only smaller trends or groupings among three or four consecutive or near consecutive layers can be seen.

$\mathrm{Al}_{2} \mathrm{O}_{3}$ and $\mathrm{CaO}$ vs. depth display similar relationships with the exception of layer $\mathrm{H}$, which is depleted in $\mathrm{CaO}$ relative to the other layers. The diagram of $\mathrm{FeO}$ vs. depth displays several groupings, from the less evolved magmas of layers $\mathrm{L}$ and $\mathrm{E}$ to the more evolved magmas of layers $\mathrm{K}$ and $\mathrm{H}$ and layers $\mathrm{D}, \mathrm{C}$, and $\mathrm{A}$, respectively. Silica has similar trends, suggesting that the ashes in each group become more silicic with time.

Ratios of the major oxides vs. depth also show groupings of tephra layers with similar element ratios. $\mathrm{CaO} / \mathrm{FeO}$ vs. depth best displays the three groupings. Within each group there is a decrease in the mafic character of the magmas with respect to time, 
Table 2. Percentage composition of major oxides from microprobe measurements of glass shards from Site 758.

\begin{tabular}{|c|c|c|c|c|c|c|c|c|c|c|c|c|c|c|}
\hline $\begin{array}{l}\text { Sample } \\
\text { Layer } \\
\text { Shard morphology }\end{array}$ & $\begin{array}{c}758 \mathrm{~A}-1 \mathrm{H}-2 \\
25 \mathrm{~cm} \\
\mathrm{~A} \\
?\end{array}$ & $\begin{array}{c}758 \mathrm{~A}-1 \mathrm{H}-2, \\
25 \mathrm{~cm} \\
\text { A } \\
\text { Platy }\end{array}$ & $\begin{array}{c}758 \mathrm{~A}-1 \mathrm{H}-2, \\
25 \mathrm{~cm} \\
\text { A } \\
\text { Platy }\end{array}$ & $\begin{array}{c}758 \mathrm{~A}-1 \mathrm{H}-2, \\
25 \mathrm{~cm} \\
\mathrm{~A} \\
\text { Bubble wall }\end{array}$ & $\begin{array}{c}758 \mathrm{~A}-1 \mathrm{H}-2, \\
25 \mathrm{~cm} \\
\text { A } \\
\text { Blocky }\end{array}$ & $\begin{array}{c}758 \mathrm{~A}-1 \mathrm{H}-2, \\
25 \mathrm{~cm} \\
\mathrm{~A} \\
\text { Bubble wall }\end{array}$ & $\begin{array}{c}758 \mathrm{~A}-1 \mathrm{H}-2 \\
25 \mathrm{~cm} \\
\text { A } \\
\text { Blocky }\end{array}$ & $\begin{array}{c}758 \mathrm{~A}-1 \mathrm{H}-2, \\
25 \mathrm{~cm} \\
\text { A } \\
\text { Blocky }\end{array}$ & $\begin{array}{c}758 \mathrm{~A}-1 \mathrm{H}-2, \\
25 \mathrm{~cm} \\
\text { A } \\
\text { Platy }\end{array}$ & $\begin{array}{c}758 \mathrm{~A}-1 \mathrm{H}-2 \\
25 \mathrm{~cm} \\
\text { A } \\
\text { Blocky }\end{array}$ & $\begin{array}{c}758 \mathrm{~A}-1 \mathrm{H}-2, \\
25 \mathrm{~cm} \\
\mathrm{~A} \\
\text { Bubble wall }\end{array}$ & $\begin{array}{c}758 \mathrm{~A}-1 \mathrm{H}-2, \\
25 \mathrm{~cm} \\
\mathrm{~A} \\
\text { Bubble wall }\end{array}$ & $\begin{array}{c}758 \mathrm{~A}-1 \mathrm{H}-2, \\
25 \mathrm{~cm} \\
\mathrm{~A} \\
\text { Bubble wall }\end{array}$ & $\begin{array}{c}758 \mathrm{~A}-1 \mathrm{H}-2 . \\
25 \mathrm{~cm} \\
\text { A } \\
\text { Bubble wall }\end{array}$ \\
\hline $\mathrm{SiO}_{2}$ & 75.02 & 74.53 & 72.93 & 72.30 & 74.39 & 73.63 & 74.87 & 73.63 & 74.59 & 73.73 & 74.61 & 73.81 & 74.29 & 74.12 \\
\hline $\mathrm{Al}_{2} \mathrm{O}_{3}$ & 12.19 & 12.07 & 12.15 & 11.87 & 12.09 & 12.11 & 11.85 & 12.19 & 11.80 & 12.06 & 11.89 & 12.04 & 12.16 & 11.91 \\
\hline $\mathrm{FeO}$ & 0.83 & 0.73 & 0.80 & 0.76 & 0.79 & 0.79 & 0.79 & 0.83 & 0.74 & 0.85 & 0.76 & 0.74 & 0.81 & 0.80 \\
\hline $\mathrm{MgO}$ & 0.09 & 0.03 & 0.08 & 0.05 & 0.08 & 0.05 & 0.06 & 0.05 & 0.05 & 0.06 & 0.04 & 0.03 & 0.05 & 0.04 \\
\hline $\mathrm{CaO}$ & 0.91 & 0.69 & 0.81 & 0.78 & 0.82 & 0.79 & 0.79 & 0.84 & 0.67 & 0.76 & 0.64 & 0.66 & 0.75 & 0.78 \\
\hline $\mathrm{K}_{2} \mathrm{O}$ & 4.65 & 4.80 & 4.94 & 4.84 & 5.05 & 4.88 & 4.94 & 4.86 & 4.88 & 4.81 & 4.92 & 5.05 & 5.00 & 4.87 \\
\hline $\mathrm{Na}_{2} \mathrm{O}$ & 1.28 & 3.10 & 2.88 & 2.90 & 2.87 & 2.78 & 2.72 & 3.04 & 3.14 & 2.83 & 3.30 & 3.06 & 3.14 & 2.97 \\
\hline $\mathrm{TiO}_{2}$ & 0.10 & 0.08 & 0.05 & 0.07 & 0.07 & 0.08 & 0.08 & 0.07 & 0.06 & 0.05 & 0.08 & 0.06 & 0.07 & 0.09 \\
\hline $\mathrm{P}_{2} \mathrm{O}_{5}$ & 0.03 & 0.00 & 0.02 & 0.01 & 0.02 & 0.01 & 0.02 & 0.04 & 0.02 & 0.03 & 0.02 & 0.01 & 0.02 & 0.02 \\
\hline Total & 95.10 & 96.03 & 94.66 & 93.58 & 96.18 & 95.12 & 96.12 & 95.55 & 95.95 & 95.18 & 96.26 & 95.46 & 96.29 & 95.60 \\
\hline $\mathrm{H}_{2} \mathrm{O}$ & 4.90 & 3.97 & 5.34 & 6.42 & 3.82 & 4.88 & 3.88 & 4.45 & 4.05 & 4.82 & 3.74 & 4.54 & 3.71 & 4.40 \\
\hline
\end{tabular}

Note: The inferred percent $\mathrm{H}_{2} \mathrm{O}$ is determined by the sum of the oxides subtracted from $100 . ?=$ the shard form does not fall into the three categories.

\begin{tabular}{|c|c|c|c|c|c|c|c|c|c|c|c|c|c|c|}
\hline $\begin{array}{l}\text { Sample } \\
\text { Layer } \\
\text { Shard morphology }\end{array}$ & $\begin{array}{c}758 \mathrm{~A}-1 \mathrm{H}-2, \\
25 \mathrm{~cm} \\
\text { A } \\
\text { Blocky }\end{array}$ & $\begin{array}{c}758 \mathrm{~A}-1 \mathrm{H}-2, \\
25 \mathrm{~cm} \\
\text { A } \\
\text { Bubble wall }\end{array}$ & $\begin{array}{c}758 \mathrm{~A}-1 \mathrm{H}-2 \\
25 \mathrm{~cm} \\
\mathrm{~A} \\
\text { Bubble wall }\end{array}$ & $\begin{array}{c}758 \mathrm{~A}-1 \mathrm{H}-2, \\
25 \mathrm{~cm} \\
\text { A } \\
\text { Platy }\end{array}$ & $\begin{array}{c}758 \mathrm{~A}-1 \mathrm{H}-2, \\
25 \mathrm{~cm} \\
\text { A } \\
\text { Platy }\end{array}$ & $\begin{array}{c}758 \mathrm{~A}-1 \mathrm{H}-2, \\
25 \mathrm{~cm} \\
\text { A } \\
\text { Blocky }\end{array}$ & $\begin{array}{c}758 \mathrm{~A}-1 \mathrm{H}-2, \\
25 \mathrm{~cm} \\
\text { A } \\
\text { Platy }\end{array}$ & $\begin{array}{c}758 \mathrm{~A}-1 \mathrm{H}-2, \\
25 \mathrm{~cm} \\
\mathrm{~A} \\
\text { Bubble wall }\end{array}$ & $\begin{array}{c}758 \mathrm{~A}-1 \mathrm{H}-2, \\
25 \mathrm{~cm} \\
\text { A } \\
\text { Blocky }\end{array}$ & $\begin{array}{c}\text { 758A-1H-2, } \\
25 \mathrm{~cm} \\
\text { A } \\
\text { Blocky }\end{array}$ & $\begin{array}{c}\text { 758A-1H-2, } \\
5 \mathrm{~cm} \\
\text { A } \\
\text { Blocky }\end{array}$ & $\begin{array}{c}758 \mathrm{~A}-1 \mathrm{H}-2 \\
5 \mathrm{~cm} \\
\mathrm{~A} \\
?\end{array}$ & $\begin{array}{c}\text { 758A-1H-2, } \\
5 \mathrm{~cm} \\
\text { A } \\
\text { Blocky }\end{array}$ & $\begin{array}{c}758 \mathrm{~A}-1 \mathrm{H}-2, \\
5 \mathrm{~cm} \\
\text { A } \\
\text { Blocky }\end{array}$ \\
\hline $\mathrm{SiO}_{2}$ & 74.31 & 74.01 & 74.79 & 75.50 & 74.43 & 75.11 & 74.53 & 74.13 & 74.78 & 74.30 & 71.98 & 73.40 & 74.83 & 74.70 \\
\hline $\mathrm{Al}_{2} \mathrm{O}_{3}$ & 12.05 & 12.10 & 12.17 & 11.96 & 12.13 & 11.84 & 11.90 & 12.18 & 12.31 & 11.87 & 11.96 & 11.74 & 11.75 & 11.93 \\
\hline $\mathrm{FeO}$ & 0.80 & 0.79 & 0.78 & 0.77 & 0.88 & 0.76 & 0.78 & 0.83 & 0.88 & 0.77 & 0.92 & $\begin{array}{r}0.74 \\
0.78\end{array}$ & 0.69 & 0.81 \\
\hline $\mathrm{MgO}$ & 0.04 & 0.05 & 0.06 & 0.03 & 0.08 & 0.04 & 0.03 & 0.08 & 0.06 & 0.06 & 0.08 & 0.02 & 0.03 & 0.04 \\
\hline $\mathrm{CaO}$ & 0.74 & 0.83 & 0.75 & 0.76 & 0.79 & 0.69 & 0.70 & 0.78 & 0.89 & 0.67 & 0.91 & 0.65 & 0.69 & 0.83 \\
\hline $\mathrm{K}_{2} \mathrm{O}$ & 4.78 & 4.89 & 5.02 & 5.07 & 4.99 & 4.87 & 4.91 & 4.81 & $\begin{array}{l}4.87 \\
4.87\end{array}$ & 4.95 & 4.76 & 5.25 & 5.08 & 5.02 \\
\hline $\mathrm{Na}_{2} \mathrm{O}$ & 3.15 & 3.10 & 2.69 & 3.03 & 2.83 & 3.15 & 2.92 & 3.04 & 3.14 & 3.18 & 2.56 & 2.93 & 2.71 & 2.85 \\
\hline $\mathrm{TiO}_{2}$ & 0.07 & 0.09 & 0.07 & 0.07 & 0.08 & 0.05 & 0.07 & 0.08 & $\begin{array}{l}0.14 \\
0.07\end{array}$ & 0.08 & 0.12 & 0.07 & 0.06 & 0.10 \\
\hline $\mathrm{P}_{2} \mathrm{O}_{5}$ & 0.01 & 0.02 & 0.01 & 0.02 & 0.02 & 0.04 & 0.02 & 0.03 & 0.03 & 0.04 & 0.02 & 0.01 & 0.00 & 0.03 \\
\hline Total & 95.95 & 95.88 & 96.34 & 97.21 & 96.23 & 96.55 & 95.86 & 95.96 & 97.03 & 95.92 & 93.31 & 94.85 & 95.84 & 96.31 \\
\hline $\mathrm{H}_{2} \mathrm{O}$ & 4.05 & $\begin{array}{r}55.100 \\
4.12\end{array}$ & $\begin{array}{r}0.54 \\
3.66\end{array}$ & $\begin{array}{r}1.21 \\
2.79\end{array}$ & $\begin{array}{r}90.23 \\
3.77\end{array}$ & $\begin{array}{r}0.53 \\
3.45\end{array}$ & $\begin{array}{r}5.80 \\
4.14\end{array}$ & $\begin{array}{r}5.90 \\
4.04\end{array}$ & $\begin{array}{r}21.03 \\
2.97\end{array}$ & $\begin{array}{r}5.92 \\
4.08\end{array}$ & $\begin{array}{r}93.311 \\
6.69\end{array}$ & $\begin{array}{r}94.83 \\
5.15\end{array}$ & $\begin{array}{r}53.84 \\
4.16\end{array}$ & $\begin{array}{r}90.31 \\
3.69\end{array}$ \\
\hline
\end{tabular}

\begin{tabular}{|c|c|c|c|c|c|c|c|c|c|c|c|c|c|c|}
\hline $\begin{array}{l}\text { Layer } \\
\text { Shard morphology }\end{array}$ & $\begin{array}{c}758 \mathrm{~A}-1 \mathrm{H}-2 \\
5 \mathrm{~cm} \\
\mathrm{~A} \\
?\end{array}$ & $\begin{array}{c}758 \mathrm{~A}-1 \mathrm{H}-2, \\
47 \mathrm{~cm} \\
\mathrm{~B} \\
\text { Bubble wall }\end{array}$ & $\begin{array}{c}758 \mathrm{~A}-1 \mathrm{H}-2, \\
47 \mathrm{~cm} \\
\mathrm{~B} \\
\text { Bubble wall }\end{array}$ & $\begin{array}{c}758 \mathrm{~A}-1 \mathrm{H}-2, \\
47 \mathrm{~cm} \\
\mathrm{~B} \\
\text { Bubble wall }\end{array}$ & $\begin{array}{c}758 \mathrm{~A}-1 \mathrm{H}-2, \\
47 \mathrm{~cm} \\
\mathrm{~B} \\
\text { Bubble wall }\end{array}$ & $\begin{array}{c}758 \mathrm{~A}-1 \mathrm{H}-2 \\
47 \mathrm{~cm} \\
\text { B } \\
\text { Blocky }\end{array}$ & $\begin{array}{c}758 \mathrm{~A}-1 \mathrm{H}-2 \\
47 \mathrm{~cm} \\
\text { B } \\
\text { Blocky }\end{array}$ & $\begin{array}{c}758 \mathrm{~A}-1 \mathrm{H}-2 \\
47 \mathrm{~cm} \\
\text { B } \\
\text { Platy }\end{array}$ & $\begin{array}{c}758 \mathrm{~A}-1 \mathrm{H}-2, \\
47 \mathrm{~cm} \\
\mathrm{~B} \\
\text { Bubble wall }\end{array}$ & $\begin{array}{c}758 \mathrm{~A}-1 \mathrm{H}-2 \\
47 \mathrm{~cm} \\
\text { B } \\
\text { Blocky }\end{array}$ & $\begin{array}{c}758 \mathrm{~A}-2 \mathrm{H}-1, \\
114 \mathrm{~cm} \\
\mathrm{C} \\
\text { Bubble wall }\end{array}$ & $\begin{array}{c}758 \mathrm{~A}-2 \mathrm{H}-1, \\
114 \mathrm{~cm} \\
\mathrm{C} \\
\text { Blocky }\end{array}$ & $\begin{array}{c}758 \mathrm{~A}-2 \mathrm{H}-1, \\
114 \mathrm{~cm} \\
\mathrm{C} \\
\text { Platy }\end{array}$ & $\begin{array}{c}758 \mathrm{~A}-2 \mathrm{H}-1, \\
114 \mathrm{~cm} \\
\mathrm{C} \\
\text { Platy }\end{array}$ \\
\hline $\mathrm{SiO}_{2}$ & 74.68 & 74.22 & 70.20 & 72.31 & 72.45 & 74.84 & 73.19 & 73.01 & 71.36 & 71.50 & 72.40 & 73.40 & 72.08 & 72.24 \\
\hline $\mathrm{Al}_{2} \mathrm{O}_{3}$ & 11.65 & 11.77 & 11.29 & 12.01 & 11.69 & 11.95 & 11.99 & 11.82 & 11.58 & 12.05 & 11.39 & 11.75 & 11.69 & 11.64 \\
\hline $\mathrm{FeO}$ & 0.75 & 0.89 & 0.93 & 0.86 & 0.85 & 0.86 & 0.84 & 0.85 & 0.79 & 0.92 & 0.95 & 0.94 & 0.94 & 0.94 \\
\hline $\mathrm{MgO}$ & 0.03 & 0.02 & 0.05 & 0.01 & 0.02 & 0.01 & 0.02 & 0.04 & 0.04 & 0.04 & 0.03 & 0.01 & 0.01 & 0.00 \\
\hline $\mathrm{CaO}$ & 0.89 & 0.79 & 0.65 & 0.79 & 0.72 & 0.70 & 0.75 & 0.83 & 0.64 & 0.96 & 0.77 & 0.63 & 0.58 & 0.48 \\
\hline $\mathrm{K}_{2} \mathrm{O}$ & 5.23 & 4.93 & 4.73 & 4.79 & 4.63 & 5.16 & 4.85 & 4.80 & 4.68 & 4.69 & 4.81 & 5.04 & 4.57 & 4.66 \\
\hline $\mathrm{Na}_{2} \mathrm{O}$ & 2.46 & 2.78 & 3.10 & 2.92 & 3.32 & 3.30 & 3.41 & 3.26 & 3.21 & 2.88 & 2.81 & 3.18 & 3.19 & 3.58 \\
\hline $\mathrm{TiO}_{2}$ & 0.09 & 0.05 & 0.05 & 0.08 & 0.09 & 0.06 & 0.06 & 0.09 & 0.07 & 0.09 & 0.09 & 0.04 & 0.05 & 0.03 \\
\hline $\mathrm{P}_{2} \mathrm{O}_{5}$ & 0.01 & 0.00 & 0.01 & 0.00 & 0.01 & 0.00 & 0.00 & 0.00 & 0.02 & 0.02 & 0.00 & 0.01 & 0.00 & 0.01 \\
\hline Total & 95.79 & 95.45 & 91.01 & 93.77 & 93.78 & 96.88 & 95.11 & 94.70 & 92.39 & 93.15 & 93.25 & 95.00 & 93.11 & 93.58 \\
\hline $\mathrm{H}_{2} \mathrm{O}$ & 4.21 & 4.55 & 8.99 & 6.23 & 6.22 & 3.12 & 4.89 & 5.30 & 7.61 & 6.85 & 6.75 & 5.00 & 6.89 & 6.42 \\
\hline
\end{tabular}




\begin{tabular}{|c|c|c|c|c|c|c|c|c|c|c|c|c|c|c|}
\hline $\begin{array}{l}\text { Sample } \\
\text { Layer } \\
\text { Shard morphology }\end{array}$ & $\begin{array}{c}758 \mathrm{~A}-2 \mathrm{H}-1, \\
114 \mathrm{~cm} \\
\text { C } \\
\text { Blocky }\end{array}$ & $\begin{array}{c}758 \mathrm{~A}-2 \mathrm{H}-1 \\
114 \mathrm{~cm} \\
\mathrm{C} \\
\text { Blocky }\end{array}$ & $\begin{array}{c}758 \mathrm{~A}-2 \mathrm{H}-1 \\
114 \mathrm{~cm} \\
\mathrm{C} \\
\text { Platy }\end{array}$ & $\begin{array}{c}758 \mathrm{~A}-2 \mathrm{H}-1, \\
114 \mathrm{~cm} \\
\text { C } \\
\text { Platy }\end{array}$ & $\begin{array}{l}758 \mathrm{~A}-2 \mathrm{H}-1, \\
114 \mathrm{~cm} \\
\mathrm{C} \\
\text { Bubble wall }\end{array}$ & $\begin{array}{c}758 \mathrm{~A}-2 \mathrm{H}-1 . \\
114 \mathrm{~cm} \\
\mathrm{C} \\
\text { Bubble wall }\end{array}$ & $\begin{array}{c}758 \mathrm{~A}-2 \mathrm{H}-1, \\
114 \mathrm{~cm} \\
\text { C } \\
\text { Blocky }\end{array}$ & $\begin{array}{l}758 \mathrm{~A}-2 \mathrm{H}-1 \\
114 \mathrm{~cm} \\
\mathrm{C} \\
\text { Bubble wall }\end{array}$ & $\begin{array}{c}758 \mathrm{~A}-2 \mathrm{H}-4, \\
38 \mathrm{~cm} \\
\mathrm{D} \\
\text { Bubble wall }\end{array}$ & $\begin{array}{c}758 \mathrm{~A}-2 \mathrm{H}-4, \\
38 \mathrm{~cm} \\
\text { D } \\
\text { Bubble wall }\end{array}$ & $\begin{array}{c}758 \mathrm{~A}-2 \mathrm{H}-4, \\
38 \mathrm{~cm} \\
\text { D } \\
\text { Blocky }\end{array}$ & $\begin{array}{c}758 \mathrm{~A}-2 \mathrm{H}-4, \\
38 \mathrm{~cm} \\
\text { D } \\
\text { Blocky }\end{array}$ & $\begin{array}{c}758 \mathrm{~A}-2 \mathrm{H}-4, \\
38 \mathrm{~cm} \\
\text { D } \\
\text { Platy }\end{array}$ & $\begin{array}{c}758 \mathrm{~A}-2 \mathrm{H}-4, \\
38 \mathrm{~cm} \\
\text { D } \\
\text { Blocky }\end{array}$ \\
\hline $\mathrm{SiO}_{2}$ & 72.39 & 71.93 & 72.87 & 72.34 & 72.83 & 71.36 & 72.26 & 72.75 & 73.06 & 73.21 & 73.52 & 73.39 & 73.97 & 74.43 \\
\hline $\mathrm{Al}_{2} \mathrm{O}_{3}$ & 11.53 & 11.55 & 11.74 & 11.45 & 11.58 & 11.52 & 11.41 & 11.81 & 11.72 & 11.78 & 11.90 & 11.76 & 11.94 & 11.66 \\
\hline $\mathrm{FeO}$ & 0.96 & 0.92 & 0.90 & 0.95 & 0.93 & 0.95 & 0.93 & 0.91 & 0.80 & 0.87 & 0.82 & 0.81 & 0.88 & 0.78 \\
\hline $\mathrm{MgO}$ & 0.00 & 0.01 & 0.00 & 0.00 & 0.00 & 0.02 & 0.02 & 0.01 & 0.03 & 0.02 & 0.02 & 0.01 & 0.03 & 0.01 \\
\hline $\mathrm{CaO}$ & 0.55 & 0.65 & 0.49 & 0.53 & 0.54 & 0.65 & 0.82 & 0.64 & 0.74 & 0.79 & 0.80 & 0.74 & 0.77 & 0.75 \\
\hline $\mathrm{K}_{2} \mathrm{O}$ & 5.04 & 4.89 & 4.59 & 4.65 & 4.49 & 4.88 & 4.65 & 4.99 & 5.10 & 5.01 & 4.93 & 4.88 & 5.05 & 4.81 \\
\hline $\mathrm{Na}_{2} \mathrm{O}$ & 3.03 & 3.12 & 3.58 & 3.32 & 3.35 & 3.05 & 3.03 & 3.06 & 2.87 & 2.76 & 3.06 & 2.95 & 2.67 & 3.04 \\
\hline $\mathrm{TiO}_{2}$ & 0.04 & 0.07 & 0.05 & 0.03 & 0.06 & 0.06 & 0.09 & 0.07 & 0.06 & 0.07 & 0.08 & 0.06 & 0.07 & 0.05 \\
\hline $\mathrm{P}_{2} \mathrm{O}_{5}$ & 0.00 & 0.00 & 0.03 & 0.01 & 0.00 & 0.00 & 0.00 & 0.02 & 0.01 & 0.00 & 0.00 & 0.01 & 0.00 & 0.01 \\
\hline Total & 93.54 & 93.14 & 94.25 & 93.28 & 93.78 & 92.49 & 93.21 & 94.26 & 94.39 & 94.51 & 95.13 & 94.61 & 95.38 & 95.54 \\
\hline $\mathrm{H}_{2} \mathrm{O}$ & 6.46 & 6.86 & 5.75 & 6.72 & 6.22 & 7.51 & 6.79 & 5.74 & 5.61 & 5.49 & 4.87 & 5.39 & 4.62 & 4.46 \\
\hline
\end{tabular}

\begin{tabular}{|c|c|c|c|c|c|c|c|c|c|c|c|c|c|c|}
\hline $\begin{array}{l}\text { Sample } \\
\text { Layer } \\
\text { Shard morphology }\end{array}$ & $\begin{array}{c}758 \mathrm{~A}-2 \mathrm{H}-4, \\
38 \mathrm{~cm} \\
\text { D } \\
\text { Platy }\end{array}$ & $\begin{array}{c}758 \mathrm{~A}-2 \mathrm{H}-4 \\
38 \mathrm{~cm} \\
\mathrm{D} \\
\text { Platy }\end{array}$ & $\begin{array}{c}758 \mathrm{~A}-2 \mathrm{H}-4, \\
38 \mathrm{~cm} \\
\mathrm{D} \\
\text { Bubble wall }\end{array}$ & $\begin{array}{c}758 \mathrm{~A}-2 \mathrm{H}-4, \\
38 \mathrm{~cm} \\
\mathrm{D} \\
\text { Bubble wall }\end{array}$ & $\begin{array}{c}758 \mathrm{~A}-2 \mathrm{H}-4 \\
38 \mathrm{~cm} \\
\mathrm{D} \\
\text { Blocky }\end{array}$ & $\begin{array}{c}758 \mathrm{~A}-2 \mathrm{H}-4, \\
38 \mathrm{~cm} \\
\mathrm{D} \\
\text { Bubble wall }\end{array}$ & $\begin{array}{c}758 \mathrm{~A}-2 \mathrm{H}-4, \\
38 \mathrm{~cm} \\
\mathrm{D} \\
\text { Blocky }\end{array}$ & $\begin{array}{c}758 \mathrm{~A}-2 \mathrm{H}-4 \\
115 \mathrm{~cm} \\
\mathrm{E} \\
\text { Bubble wall }\end{array}$ & $\begin{array}{c}758 \mathrm{~A}-2 \mathrm{H}-4 \\
115 \mathrm{~cm} \\
\mathrm{E} \\
\text { Platy }\end{array}$ & $\begin{array}{c}758 \mathrm{~A}-2 \mathrm{H}-4, \\
115 \mathrm{~cm} \\
\mathrm{E} \\
\text { Bubble wall }\end{array}$ & $\begin{array}{c}758 \mathrm{~A}-2 \mathrm{H}-4, \\
115 \mathrm{~cm} \\
\mathrm{E} \\
\text { Blocky }\end{array}$ & $\begin{array}{c}758 \mathrm{~A}-2 \mathrm{H}-4, \\
115 \mathrm{~cm} \\
\mathrm{E} \\
\text { Bubble wall }\end{array}$ & $\begin{array}{c}758 \mathrm{~A}-2 \mathrm{H}-4 \\
115 \mathrm{~cm} \\
\mathrm{E} \\
\text { Blocky }\end{array}$ & $\begin{array}{c}758 \mathrm{~A}-2 \mathrm{H}-4, \\
115 \mathrm{~cm} \\
\mathrm{E} \\
\text { Platy }\end{array}$ \\
\hline $\mathrm{SiO}_{2}$ & 73.88 & 74.04 & 73.76 & 73.62 & 74.09 & 73.33 & 76.05 & 72.26 & 71.85 & 71.42 & 72.27 & 70.33 & 72.01 & 71.13 \\
\hline $\mathrm{Al}_{2} \mathrm{O}_{3}$ & 11.69 & 11.58 & 11.82 & 11.84 & 11.57 & 11.67 & 11.92 & 12.27 & 12.38 & 12.53 & 12.37 & 12.85 & 12.42 & 12.34 \\
\hline $\mathrm{FeO}$ & 0.84 & 0.84 & 0.79 & 0.80 & 0.86 & 0.82 & 0.87 & 1.15 & 1.10 & 1.15 & 1.16 & 1.22 & 1.15 & 1.10 \\
\hline $\mathrm{MgO}$ & 0.01 & 0.01 & 0.01 & 0.03 & 0.00 & 0.03 & 0.06 & 0.11 & 0.09 & 0.09 & 0.14 & 0.15 & 0.11 & 0.11 \\
\hline $\mathrm{CaO}$ & 0.79 & 0.67 & 0.67 & 0.78 & 0.69 & 0.74 & 0.78 & 0.82 & 0.84 & 0.85 & 0.84 & 0.97 & 0.90 & 0.83 \\
\hline $\mathrm{K}_{2} \mathrm{O}$ & 4.98 & 5.01 & 5.29 & 5.02 & 4.74 & 4.84 & 4.50 & 3.90 & 3.96 & 3.98 & 3.90 & 3.83 & 4.01 & 4.18 \\
\hline $\mathrm{Na}_{2} \mathrm{O}$ & 2.83 & 2.98 & 2.80 & 3.10 & 3.21 & 2.83 & 0.92 & 3.88 & 3.69 & 3.84 & 3.68 & 3.94 & 3.50 & 3.89 \\
\hline $\mathrm{TiO}_{2}$ & 0.07 & 0.08 & 0.08 & 0.05 & 0.05 & 0.06 & 0.11 & 0.12 & 0.14 & 0.14 & 0.14 & 0.18 & 0.14 & 0.11 \\
\hline $\mathrm{P}_{2} \mathrm{O}_{5}$ & 0.01 & 0.02 & 0.00 & 0.00 & 0.01 & 0.02 & 0.00 & 0.03 & 0.01 & 0.01 & 0.04 & 0.03 & 0.03 & 0.03 \\
\hline Total & 95.10 & 95.23 & 95.22 & 95.24 & 95.22 & 94.34 & 95.21 & 94.54 & 94.06 & 94.01 & 94.54 & 93.50 & 94.27 & 93.72 \\
\hline $\mathrm{H}_{2} \mathrm{O}$ & 4.90 & 4.77 & 4.78 & 4.76 & 4.78 & 5.66 & 4.79 & 5.46 & 5.94 & 5.99 & 5.46 & 6.50 & 5.73 & 6.28 \\
\hline
\end{tabular}

\begin{tabular}{|c|c|c|c|c|c|c|c|c|c|c|c|c|c|c|}
\hline $\begin{array}{l}\text { Sample } \\
\text { Layer } \\
\text { Shard morphology }\end{array}$ & $\begin{array}{c}758 \mathrm{~A}-2 \mathrm{H}-4, \\
115 \mathrm{~cm} \\
\mathrm{E} \\
?\end{array}$ & $\begin{array}{c}758 \mathrm{~A}-2 \mathrm{H}-4, \\
115 \mathrm{~cm} \\
\mathrm{E} \\
\text { Blocky }\end{array}$ & $\begin{array}{c}758 \mathrm{~A}-2 \mathrm{H}-4 \\
115 \mathrm{~cm} \\
\mathrm{E} \\
\text { Bubble wall }\end{array}$ & $\begin{array}{c}758 \mathrm{~A}-2 \mathrm{H}-4 \\
115 \mathrm{~cm} \\
\mathrm{E} \\
\text { Bubble wall }\end{array}$ & $\begin{array}{c}758 \mathrm{~A}-3 \mathrm{H}-3 \\
125 \mathrm{~cm} \\
\text { F } \\
\text { Platy }\end{array}$ & $\begin{array}{c}758 \mathrm{~A}-3 \mathrm{H}-3 \\
125 \mathrm{~cm} \\
\mathrm{~F} \\
\text { Blocky }\end{array}$ & $\begin{array}{c}758 \mathrm{~A}-3 \mathrm{H}-3, \\
125 \mathrm{~cm} \\
\mathrm{~F} \\
\text { Bubble wall }\end{array}$ & $\begin{array}{c}758 \mathrm{~A}-3 \mathrm{H}-3 \\
125 \mathrm{~cm} \\
\text { F } \\
\text { Platy }\end{array}$ & $\begin{array}{c}758 \mathrm{~A}-3 \mathrm{H}-3, \\
125 \mathrm{~cm} \\
\mathrm{~F} \\
\text { Blocky }\end{array}$ & $\begin{array}{c}758 \mathrm{~A}-3 \mathrm{H}-3, \\
125 \mathrm{~cm} \\
\text { F } \\
\text { Platy }\end{array}$ & $\begin{array}{c}758 \mathrm{~A}-3 \mathrm{H}-3, \\
125 \mathrm{~cm} \\
\mathrm{~F} \\
\text { Bubble wall }\end{array}$ & $\begin{array}{c}758 \mathrm{~A}-3 \mathrm{H}-3, \\
125 \mathrm{~cm} \\
\mathrm{~F} \\
\text { Blocky }\end{array}$ & $\begin{array}{c}758 \mathrm{~A}-3 \mathrm{H}-3, \\
125 \mathrm{~cm} \\
\mathrm{~F} \\
\text { Bubble wall }\end{array}$ & $\begin{array}{c}758 \mathrm{~A}-4 \mathrm{H}-5, \\
3 \mathrm{~cm} \\
\mathrm{H} \\
\text { Bubble wall }\end{array}$ \\
\hline $\mathrm{SiO}_{2}$ & 71.75 & 72.25 & 71.80 & 70.68 & 73.56 & 73.56 & 72.77 & 72.29 & 74.00 & 72.98 & 71.25 & 72.89 & 72.95 & 71.91 \\
\hline $\mathrm{Al}_{2} \mathrm{O}_{3}$ & 12.37 & 12.52 & 12.39 & 12.16 & 11.72 & 11.42 & 11.92 & 11.85 & 11.86 & 11.71 & 12.14 & 11.67 & 11.57 & 11.47 \\
\hline $\mathrm{FeO}$ & 1.17 & 1.08 & 1.12 & 1.14 & 1.03 & 1.06 & 1.08 & 1.01 & 1.09 & 1.03 & 1.30 & 1.04 & 1.07 & 0.76 \\
\hline $\mathrm{MgO}$ & 0.11 & 0.13 & 0.09 & 0.10 & 0.00 & 0.01 & 0.01 & 0.02 & 0.04 & 0.00 & 0.03 & 0.01 & 0.00 & 0.02 \\
\hline $\mathrm{CaO}$ & 0.87 & 0.87 & 0.84 & 0.86 & 0.83 & 0.84 & 0.72 & 0.84 & 1.07 & 0.82 & 0.99 & 0.76 & 0.73 & 0.48 \\
\hline $\mathrm{K}_{2} \mathrm{O}$ & 4.09 & 3.85 & 4.02 & 3.87 & 4.73 & 4.79 & 4.60 & 4.65 & 4.16 & 4.69 & 5.04 & 4.62 & 4.74 & 4.38 \\
\hline $\mathrm{Na}_{2} \mathrm{O}$ & 3.77 & 3.09 & 3.90 & 3.79 & 2.93 & 2.80 & 3.16 & 2.87 & 2.49 & 2.86 & 2.90 & 3.18 & 3.01 & 3.53 \\
\hline $\mathrm{TiO}_{2}$ & 0.13 & 0.12 & 0.13 & 0.10 & 0.03 & 0.05 & 0.07 & 0.05 & 0.03 & 0.06 & 0.08 & 0.04 & 0.05 & 0.04 \\
\hline $\mathrm{P}_{2} \mathrm{O}_{5}$ & 0.04 & 0.02 & 0.02 & 0.02 & 0.01 & 0.03 & 0.02 & 0.02 & 0.00 & 0.00 & 0.02 & 0.00 & 0.03 & 0.02 \\
\hline Total & 94.30 & 93.93 & 94.31 & 92.72 & 94.84 & 94.56 & 94.35 & 93.60 & 94.74 & 94.15 & 93.75 & 94.21 & 94.15 & 92.61 \\
\hline $\mathrm{H}_{2} \mathrm{O}$ & 5.70 & 6.07 & 5.69 & 7.28 & 5.16 & 5.44 & 5.65 & 6.40 & 5.26 & 5.85 & 6.25 & 5.79 & 5.85 & 7.39 \\
\hline
\end{tabular}


Table 2 (continued).

\begin{tabular}{|c|c|c|c|c|c|c|c|c|c|c|c|c|c|c|}
\hline $\begin{array}{l}\text { Sample } \\
\text { Layer } \\
\text { Shard morphology }\end{array}$ & $\begin{array}{c}758 \mathrm{~A}-4 \mathrm{H}-5, \\
3 \mathrm{~cm} \\
\mathrm{H} \\
\text { Bubble wall }\end{array}$ & $\begin{array}{c}758 \mathrm{~A}-4 \mathrm{H}-5, \\
3 \mathrm{~cm} \\
\mathrm{H} \\
\text { Blocky }\end{array}$ & $\begin{array}{c}758 \mathrm{~A}-4 \mathrm{H}-5, \\
3 \mathrm{~cm} \\
\mathrm{H} \\
\text { Bubble wall }\end{array}$ & $\begin{array}{c}758 \mathrm{~A}-4 \mathrm{H}-5, \\
3 \mathrm{~cm} \\
\mathrm{H} \\
\text { Bubble wall }\end{array}$ & $\begin{array}{c}758 \mathrm{~A}-4 \mathrm{H}-5, \\
3 \mathrm{~cm} \\
\mathrm{H} \\
\text { Blocky }\end{array}$ & $\begin{array}{c}758 \mathrm{~A}-4 \mathrm{H}-5 \\
3 \mathrm{~cm} \\
\mathrm{H} \\
\text { Blocky }\end{array}$ & $\begin{array}{c}758 \mathrm{~A}-4 \mathrm{H}-5, \\
3 \mathrm{~cm} \\
\mathrm{H} \\
\text { Bubble wall }\end{array}$ & $\begin{array}{c}758 \mathrm{~A}-7 \mathrm{H}-2, \\
120 \mathrm{~cm} \\
1 \\
\text { Blocky }\end{array}$ & $\begin{array}{c}758 \mathrm{~A}-7 \mathrm{H}-2, \\
120 \mathrm{~cm} \\
1 \\
\text { Bubble wall }\end{array}$ & $\begin{array}{c}758 \mathrm{~A}-7 \mathrm{H}-2, \\
120 \mathrm{~cm} \\
1 \\
\text { Bubble wall }\end{array}$ & $\begin{array}{c}758 \mathrm{~A}-7 \mathrm{H}-2, \\
120 \mathrm{~cm} \\
1 \\
\text { Bubble wall }\end{array}$ & $\begin{array}{c}758 \mathrm{~A}-7 \mathrm{H}-2 \\
120 \mathrm{~cm} \\
1 \\
\text { Platy }\end{array}$ & $\begin{array}{c}758 \mathrm{~A}-7 \mathrm{H}-2, \\
120 \mathrm{~cm} \\
1 \\
\text { Bubble wall }\end{array}$ & $\begin{array}{c}758 \mathrm{~B}-1 \mathrm{H}-1, \\
114 \mathrm{~cm} \\
\text { A } \\
\text { Blocky }\end{array}$ \\
\hline $\mathrm{SiO}_{2}$ & 71.66 & 72.90 & 72.73 & 73.89 & 72.33 & 71.69 & 72.76 & 74.24 & 74.95 & 73.90 & 72.49 & 72.56 & 72.88 & 75.22 \\
\hline $\mathrm{Al}_{2} \mathrm{O}_{3}$ & 11.30 & 11.68 & 11.85 & 11.75 & 11.55 & 11.60 & 11.34 & 12.01 & 12.06 & 11.84 & 11.86 & 11.69 & 12.32 & 11.92 \\
\hline $\mathrm{FeO}$ & 0.67 & 0.71 & 0.74 & 0.74 & 0.74 & 0.73 & 0.73 & 0.58 & 0.63 & 0.59 & 0.67 & 0.61 & 0.90 & 0.74 \\
\hline $\mathrm{MgO}$ & 0.03 & 0.02 & 0.02 & 0.01 & 0.03 & 0.02 & 0.04 & 0.02 & 0.04 & 0.02 & 0.03 & 0.03 & 0.13 & 0.04 \\
\hline $\mathrm{CaO}$ & 0.44 & 0.47 & 0.43 & 0.42 & 0.42 & 0.49 & 0.44 & 0.60 & 0.66 & 0.64 & 0.87 & 0.60 & 1.18 & 0.63 \\
\hline $\mathrm{K}_{2} \mathrm{O}$ & 4.37 & 4.00 & 4.39 & 3.85 & 4.10 & 4.35 & 4.09 & 4.52 & 4.55 & 4.67 & 3.65 & 4.37 & 3.44 & 4.85 \\
\hline $\mathrm{Na}_{2} \mathrm{O}$ & 2.83 & 3.56 & 3.50 & 3.51 & 2.94 & 3.17 & 3.60 & 2.98 & 3.11 & 3.06 & 3.57 & 2.84 & 3.32 & 3.19 \\
\hline $\mathrm{TiO}_{2}$ & 0.04 & 0.03 & 0.04 & 0.05 & 0.04 & 0.05 & 0.04 & 0.05 & 0.03 & 0.03 & 0.04 & 0.04 & 0.10 & 0.05 \\
\hline $\mathrm{P}_{2} \mathrm{O}_{5}^{2}$ & 0.02 & 0.03 & 0.02 & 0.02 & 0.01 & 0.03 & 0.01 & 0.00 & 0.00 & 0.01 & 0.01 & 0.00 & 0.03 & 0.04 \\
\hline Total & 91.36 & 93.40 & 93.72 & 94.24 & 92.16 & 92.13 & 93.05 & 95.00 & 96.03 & 94.76 & 93.19 & 92.74 & 94.30 & 96.68 \\
\hline $\mathrm{H}_{2} \mathrm{O}$ & 8.64 & 6.60 & 6.28 & 5.76 & 7.84 & 7.87 & 6.95 & 5.00 & 3.97 & 5.24 & 6.81 & 7.26 & 5.70 & 3.32 \\
\hline
\end{tabular}

\begin{tabular}{|c|c|c|c|c|c|c|c|c|c|c|c|c|c|c|}
\hline $\begin{array}{l}\text { Sample } \\
\text { Layer } \\
\text { Shard morphology }\end{array}$ & $\begin{array}{c}758 \mathrm{~B}-1 \mathrm{H}-1, \\
114 \mathrm{~cm} \\
\text { A } \\
\text { Platy }\end{array}$ & $\begin{array}{c}758 \mathrm{~B}-1 \mathrm{H}-1, \\
114 \mathrm{~cm} \\
\mathrm{~A} \\
?\end{array}$ & $\begin{array}{c}758 \mathrm{~B}-1 \mathrm{H}-1, \\
114 \mathrm{~cm} \\
\mathrm{~A} \\
\text { Platy }\end{array}$ & $\begin{array}{c}758 \mathrm{~B}-1 \mathrm{H}-1, \\
114 \mathrm{~cm} \\
\text { A } \\
\text { Blocky }\end{array}$ & $\begin{array}{c}758 \mathrm{~B}-1 \mathrm{H}-1 \\
114 \mathrm{~cm} \\
\mathrm{~A} \\
\text { Bubble wall }\end{array}$ & $\begin{array}{c}758 \mathrm{~B}-1 \mathrm{H}-1, \\
114 \mathrm{~cm} \\
\text { A } \\
\text { Blocky }\end{array}$ & $\begin{array}{c}758 \mathrm{~B}-1 \mathrm{H}-1, \\
114 \mathrm{~cm} \\
\text { A } \\
\text { Blocky }\end{array}$ & $\begin{array}{c}758 \mathrm{~B}-1 \mathrm{H}-1, \\
114 \mathrm{~cm} \\
\mathrm{~A} \\
?\end{array}$ & $\begin{array}{c}758 \mathrm{~B}-1 \mathrm{H}-1, \\
114 \mathrm{~cm} \\
\mathrm{~A} \\
\text { Blocky }\end{array}$ & $\begin{array}{c}758 \mathrm{~B}-1 \mathrm{H}-1, \\
114 \mathrm{~cm} \\
\text { A } \\
\text { Blocky }\end{array}$ & $\begin{array}{c}758 \mathrm{~B}-1 \mathrm{H}-1, \\
114 \mathrm{~cm} \\
\mathrm{~A} \\
\text { Blocky }\end{array}$ & $\begin{array}{c}758 \mathrm{~B}-1 \mathrm{H}-1, \\
114 \mathrm{~cm} \\
\mathrm{~A} \\
\text { Bubble wall }\end{array}$ & $\begin{array}{c}758 \mathrm{~B}-1 \mathrm{H}-1, \\
114 \mathrm{~cm} \\
\mathrm{~A} \\
\text { Bubble wall }\end{array}$ & $\begin{array}{c}758 \mathrm{~B}-1 \mathrm{H}-1, \\
114 \mathrm{~cm} \\
\text { A } \\
\text { Blocky }\end{array}$ \\
\hline $\mathrm{SiO}_{2}$ & 75.75 & 74.79 & 76.19 & 75.93 & 75.49 & 75.04 & 75.74 & 74.87 & 75.33 & 75.00 & 74.78 & 76.25 & 75.09 & 75.16 \\
\hline $\mathrm{Al}_{2} \mathrm{O}_{3}$ & 11.99 & 11.79 & 11.99 & 11.71 & 11.94 & 11.81 & 12.18 & 11.89 & 12.10 & 11.87 & 11.91 & 12.21 & 11.88 & 11.95 \\
\hline $\mathrm{FeO}$ & 0.70 & 0.78 & 0.80 & 0.75 & 0.77 & 0.80 & 0.79 & 0.77 & 0.81 & 0.81 & 0.80 & 0.78 & 0.80 & 0.76 \\
\hline $\mathrm{MgO}$ & 0.06 & 0.04 & 0.06 & 0.08 & 0.07 & 0.20 & 0.05 & 0.08 & 0.04 & 0.04 & 0.05 & 0.05 & 0.06 & 0.05 \\
\hline $\mathrm{CaO}$ & 0.61 & 0.80 & 0.79 & 0.68 & 0.79 & 0.76 & 0.76 & 0.81 & 0.76 & 0.78 & 0.78 & 0.66 & 0.84 & 0.84 \\
\hline $\mathrm{K}_{2} \mathrm{O}$ & 4.99 & 5.07 & 4.51 & 5.06 & 4.94 & 5.03 & 5.14 & 4.86 & 5.03 & 4.95 & 4.85 & 5.17 & 5.09 & 5.06 \\
\hline $\mathrm{Na}_{2} \mathrm{O}$ & 3.11 & 3.10 & 2.30 & 3.05 & 3.46 & 3.07 & 3.14 & 3.16 & 3.22 & 2.96 & 3.15 & 3.18 & 3.03 & 2.99 \\
\hline $\mathrm{TiO}_{2}$ & 0.06 & 0.08 & 0.08 & 0.07 & 0.08 & 0.10 & 0.08 & 0.08 & 0.08 & 0.08 & 0.07 & 0.08 & 0.07 & 0.09 \\
\hline $\mathrm{P}_{2} \mathrm{O}_{5}^{2}$ & 0.02 & 0.03 & 0.03 & 0.00 & 0.01 & 0.03 & 0.02 & 0.01 & 0.02 & 0.01 & 0.01 & 0.02 & 0.01 & 0.02 \\
\hline Total & 97.29 & 96.48 & 96.75 & 97.33 & 97.55 & 96.84 & 97.90 & 96.53 & 97.39 & 96.50 & 96.40 & 98.40 & 96.87 & 96.92 \\
\hline $\mathrm{H}_{2} \mathrm{O}$ & 2.71 & 3.52 & 3.25 & 2.67 & 2.45 & 3.16 & 2.10 & 3.47 & 2.61 & 3.50 & 3.60 & 1.60 & 3.13 & 3.08 \\
\hline
\end{tabular}

\begin{tabular}{|c|c|c|c|c|c|c|c|c|c|c|c|c|c|c|}
\hline $\begin{array}{l}\text { Sample } \\
\text { Layer } \\
\text { Shard morphology }\end{array}$ & $\begin{array}{c}758 \mathrm{~B}-1 \mathrm{H}-1, \\
114 \mathrm{~cm} \\
\text { A } \\
\text { Platy }\end{array}$ & $\begin{array}{c}758 \mathrm{~B}-1 \mathrm{H}-1, \\
114 \mathrm{~cm} \\
\text { A } \\
\text { Bubble wall }\end{array}$ & $\begin{array}{c}758 \mathrm{~B}-1 \mathrm{H}-1, \\
114 \mathrm{~cm} \\
\text { A } \\
\text { Platy }\end{array}$ & $\begin{array}{c}758 \mathrm{~B}-1 \mathrm{H}-1, \\
114 \mathrm{~cm} \\
\text { A } \\
\text { Blocky }\end{array}$ & $\begin{array}{l}\text { 758B-1H-1, } \\
114 \mathrm{~cm} \\
\text { A } \\
\text { Blocky }\end{array}$ & $\begin{array}{c}758 \mathrm{~B}-1 \mathrm{H}-1, \\
114 \mathrm{~cm} \\
\mathrm{~A} \\
\text { Bubble wall }\end{array}$ & $\begin{array}{c}758 \mathrm{~B}-1 \mathrm{H}-1, \\
114 \mathrm{~cm} \\
\text { A } \\
\text { Blocky }\end{array}$ & $\begin{array}{c}758 \mathrm{~B}-1 \mathrm{H}-1, \\
114 \mathrm{~cm} \\
\text { A } \\
\text { Bubble wall }\end{array}$ & $\begin{array}{c}758 \mathrm{~B}-1 \mathrm{H}-1, \\
114 \mathrm{~cm} \\
\text { A } \\
\text { Bubble wall }\end{array}$ & $\begin{array}{c}758 \mathrm{~B}-1 \mathrm{H}-1, \\
114 \mathrm{~cm} \\
\text { A } \\
\text { Blocky }\end{array}$ & $\begin{array}{c}758 \mathrm{~B}-1 \mathrm{H}-1, \\
114 \mathrm{~cm} \\
\text { A } \\
\text { Blocky }\end{array}$ & $\begin{array}{c}758 \mathrm{~B}-1 \mathrm{H}-5, \\
119 \mathrm{~cm} \\
\mathrm{C} \\
\text { Bubble wall }\end{array}$ & $\begin{array}{c}758 \mathrm{~B}-1 \mathrm{H}-5, \\
119 \mathrm{~cm} \\
\text { C } \\
\text { Platy }\end{array}$ & $\begin{array}{c}758 \mathrm{~B}-1 \mathrm{H}-5, \\
119 \mathrm{~cm} \\
\text { C } \\
\text { Blocky }\end{array}$ \\
\hline $\mathrm{SiO}_{2}$ & 74.90 & 75.13 & 74.72 & 75.39 & 74.32 & 73.60 & 74.42 & 74.74 & 74.44 & 74.50 & 75.28 & 73.32 & 74.24 & 73.24 \\
\hline $\mathrm{Al}_{2} \mathrm{O}_{3}$ & 11.74 & 11.89 & 11.99 & 11.92 & 12.02 & 12.22 & 11.90 & 11.88 & 11.99 & 12.49 & 12.21 & 11.61 & 12.02 & 11.68 \\
\hline $\mathrm{FeO}$ & 0.76 & 0.80 & 0.76 & 0.80 & 0.86 & 0.86 & 0.82 & 0.81 & 0.81 & 0.85 & 0.83 & 0.91 & 0.98 & 0.92 \\
\hline $\mathrm{MgO}$ & 0.04 & 0.05 & 0.06 & 0.07 & 0.04 & 0.05 & 0.05 & 0.04 & 0.02 & 0.03 & 0.06 & 0.03 & 0.01 & 0.04 \\
\hline $\mathrm{CaO}$ & 0.67 & 0.79 & 0.76 & 0.72 & 0.78 & 0.83 & 0.66 & 0.69 & 0.67 & 0.77 & 0.79 & 0.79 & 0.62 & 0.72 \\
\hline $\mathrm{K}_{2} \mathrm{O}$ & 5.11 & 4.88 & 5.11 & 4.90 & 4.97 & 4.91 & 4.72 & 4.66 & 4.93 & 4.79 & 4.95 & 4.72 & 4.95 & 4.93 \\
\hline $\mathrm{Na}_{2} \mathrm{O}$ & 3.23 & 2.95 & 3.04 & 3.18 & 3.29 & 2.83 & 3.21 & 3.24 & 3.37 & 3.12 & 3.05 & 2.96 & 3.19 & 3.11 \\
\hline $\mathrm{TiO}_{2}$ & 0.07 & 0.07 & 0.07 & 0.07 & 0.09 & 0.08 & 0.06 & 0.05 & 0.03 & 0.06 & 0.08 & 0.07 & 0.03 & 0.04 \\
\hline $\mathrm{P}_{2} \mathrm{O}_{5}$ & 0.04 & 0.03 & 0.06 & 0.03 & 0.02 & 0.04 & 0.05 & 0.05 & 0.06 & 0.01 & 0.03 & 0.04 & 0.01 & 0.01 \\
\hline Total & 96.56 & 96.59 & 96.57 & 97.08 & 96.39 & 95.42 & 95.89 & 96.16 & 96.32 & 96.62 & 97.28 & 94.45 & 96.05 & 94.69 \\
\hline $\mathrm{H}_{2} \mathrm{O}$ & 3.44 & 3.41 & 3.43 & 2.92 & 3.61 & 4.58 & 4.11 & 3.84 & 3.68 & 3.38 & 2.72 & 5.55 & 3.95 & 5.31 \\
\hline
\end{tabular}




\begin{tabular}{|c|c|c|c|c|c|c|c|c|c|c|c|c|c|c|}
\hline $\begin{array}{l}\text { Sample } \\
\text { Layer } \\
\text { Shard morphology }\end{array}$ & $\begin{array}{c}758 \mathrm{~B}-1 \mathrm{H}-5, \\
119 \mathrm{~cm} \\
\mathrm{C} \\
\text { Blocky }\end{array}$ & $\begin{array}{c}758 \mathrm{~B}-1 \mathrm{H}-5, \\
119 \mathrm{~cm} \\
\mathrm{C} \\
\text { Blocky }\end{array}$ & $\begin{array}{c}758 \mathrm{~B}-1 \mathrm{H}-5, \\
119 \mathrm{~cm} \\
\mathrm{C} \\
\text { Blocky }\end{array}$ & $\begin{array}{c}758 \mathrm{~B}-1 \mathrm{H}-5, \\
119 \mathrm{~cm} \\
\text { C } \\
\text { Blocky }\end{array}$ & $\begin{array}{l}758 \mathrm{~B}-1 \mathrm{H}-5, \\
119 \mathrm{~cm} \\
\mathrm{C} \\
\text { Bubble wall }\end{array}$ & $\begin{array}{c}758 \mathrm{~B}-2 \mathrm{H}-2, \\
25 \mathrm{~cm} \\
\mathrm{~d} \\
\text { Bubble wall }\end{array}$ & $\begin{array}{c}758 \mathrm{~B}-2 \mathrm{H}-2, \\
25 \mathrm{~cm} \\
\mathrm{~d} \\
\text { Bubble wall }\end{array}$ & $\begin{array}{c}758 \mathrm{~B}-2 \mathrm{H}-2, \\
25 \mathrm{~cm} \\
\mathrm{~d} \\
\text { Blocky }\end{array}$ & $\begin{array}{c}758 \mathrm{~B}-2 \mathrm{H}-2, \\
25 \mathrm{~cm} \\
\mathrm{~d} \\
\text { Bubble wall }\end{array}$ & $\begin{array}{c}758 \mathrm{~B}-2 \mathrm{H}-2 . \\
25 \mathrm{~cm} \\
\mathrm{~d} \\
\text { Bubble wall }\end{array}$ & $\begin{array}{c}758 \mathrm{~B}-2 \mathrm{H}-2, \\
25 \mathrm{~cm} \\
\mathrm{~d} \\
\text { Bubble wall }\end{array}$ & $\begin{array}{c}758 \mathrm{~B}-2 \mathrm{H}-2, \\
61 \mathrm{~cm} \\
\mathrm{E} \\
?\end{array}$ & $\begin{array}{c}758 \mathrm{~B}-2 \mathrm{H}-2, \\
61 \mathrm{~cm} \\
\mathrm{E} \\
\text { Blocky }\end{array}$ & $\begin{array}{c}758 \mathrm{~B}-2 \mathrm{H}-2, \\
61 \mathrm{~cm} \\
\mathrm{E} \\
\text { Bubble wall }\end{array}$ \\
\hline $\mathrm{SiO}_{2}$ & 73.02 & 72.40 & 73.01 & 73.05 & 73.39 & 73.02 & 73.12 & 72.07 & 72.13 & 72.14 & 72.37 & 71.72 & 72.14 & 70.00 \\
\hline $\mathrm{Al}_{2} \mathrm{O}_{3}$ & 11.66 & 11.49 & 11.55 & 11.71 & 11.91 & 11.97 & 11.64 & 11.50 & 11.48 & 11.75 & 11.59 & 12.27 & 12.21 & 12.07 \\
\hline $\mathrm{FeO}$ & 0.89 & 0.87 & 0.90 & 0.91 & 0.89 & 0.89 & 0.84 & 0.81 & 0.82 & 0.86 & 0.80 & 1.16 & 1.22 & 1.12 \\
\hline $\mathrm{MgO}$ & 0.01 & 0.04 & 0.00 & 0.03 & 0.02 & 0.02 & 0.02 & 0.02 & 0.02 & 0.01 & 0.02 & 0.09 & 0.09 & 0.12 \\
\hline $\mathrm{CaO}$ & 0.66 & 0.76 & 0.46 & 0.78 & 0.65 & 0.72 & 0.76 & 0.75 & 0.73 & 0.72 & 0.72 & 0.85 & 0.93 & 0.87 \\
\hline $\mathrm{K}_{2} \mathrm{O}$ & 4.59 & 4.51 & 4.31 & 4.64 & 4.59 & 4.70 & 4.59 & 4.55 & 4.71 & 4.47 & 4.48 & 3.99 & 3.97 & 4.00 \\
\hline $\mathrm{Na}_{2} \mathrm{O}$ & 3.01 & 2.85 & 3.65 & 3.06 & 3.30 & 3.28 & 3.23 & 3.14 & 3.14 & 3.24 & 3.29 & 3.84 & 3.82 & 3.57 \\
\hline $\mathrm{TiO}_{2}$ & 0.06 & 0.07 & 0.02 & 0.05 & 0.05 & 0.08 & 0.06 & 0.03 & 0.07 & 0.04 & 0.06 & 0.12 & 0.16 & 0.13 \\
\hline $\mathrm{P}_{2} \mathrm{O}_{5}$ & 0.01 & 0.02 & 0.00 & 0.03 & 0.01 & 0.01 & 0.01 & 0.03 & 0.03 & 0.01 & 0.04 & 0.02 & 0.05 & 0.02 \\
\hline Total & 93.91 & 93.01 & 93.90 & 94.26 & 94.81 & 94.69 & 94.27 & 92.90 & 93.13 & 93.24 & 93.37 & 94.06 & 94.59 & 91.90 \\
\hline $\mathrm{H}_{2} \mathrm{O}$ & 6.09 & 6.99 & 6.10 & 5.74 & 5.19 & 5.31 & 5.73 & 7.10 & 6.87 & 6.76 & 6.63 & 5.94 & 5.41 & 8.10 \\
\hline
\end{tabular}

\begin{tabular}{|c|c|c|c|c|c|c|c|c|c|c|c|c|c|c|}
\hline $\begin{array}{l}\text { Sample } \\
\text { Layer } \\
\text { Shard morphology }\end{array}$ & $\begin{array}{c}758 \mathrm{~B}-2 \mathrm{H}-2, \\
61 \mathrm{~cm} \\
\text { E } \\
\text { Platy }\end{array}$ & $\begin{array}{c}758 \mathrm{~B}-2 \mathrm{H}-2, \\
61 \mathrm{~cm} \\
\mathrm{E} \\
\text { Blocky }\end{array}$ & $\begin{array}{c}758 \mathrm{~B}-2 \mathrm{H}-2, \\
61 \mathrm{~cm} \\
\text { E } \\
\text { Platy }\end{array}$ & $\begin{array}{c}758 \mathrm{~B}-2 \mathrm{H}-2, \\
61 \mathrm{~cm} \\
\mathrm{E} \\
\text { Bubble wall }\end{array}$ & $\begin{array}{c}758 \mathrm{~B}-2 \mathrm{H}-2, \\
61 \mathrm{~cm} \\
\mathrm{E} \\
\text { Bubble wall }\end{array}$ & $\begin{array}{c}758 \mathrm{~B}-3 \mathrm{H}-4 \\
69 \mathrm{~cm} \\
\mathrm{G} \\
\text { Platy }\end{array}$ & $\begin{array}{c}758 \mathrm{~B}-3 \mathrm{H}-4, \\
69 \mathrm{~cm} \\
\mathrm{G} \\
?\end{array}$ & $\begin{array}{c}758 \mathrm{~B}-3 \mathrm{H}-4 \\
69 \mathrm{~cm} \\
\mathrm{G} \\
\text { Bubble wall }\end{array}$ & $\begin{array}{c}758 \mathrm{~B}-3 \mathrm{H}-4 \\
69 \mathrm{~cm} \\
\mathrm{G} \\
\text { Bubble wall }\end{array}$ & $\begin{array}{c}758 \mathrm{~B}-3 \mathrm{H}-4, \\
69 \mathrm{~cm} \\
\mathrm{G} \\
\text { Blocky }\end{array}$ & $\begin{array}{c}758 \mathrm{~B}-3 \mathrm{H}-4, \\
69 \mathrm{~cm} \\
\text { G } \\
\text { Platy }\end{array}$ & $\begin{array}{c}758 \mathrm{~B}-3 \mathrm{H}-4, \\
69 \mathrm{~cm} \\
\text { G } \\
\text { Platy }\end{array}$ & $\begin{array}{c}758 \mathrm{~B}-3 \mathrm{H}-4, \\
69 \mathrm{~cm} \\
\mathrm{G} \\
\text { Bubble wall }\end{array}$ & $\begin{array}{c}758 \mathrm{~B}-3 \mathrm{H}-4, \\
69 \mathrm{~cm} \\
\mathrm{G} \\
\text { Bubble wall }\end{array}$ \\
\hline $\mathrm{SiO}_{2}$ & 70.74 & 72.09 & 71.13 & 71.49 & 71.56 & 73.23 & 72.50 & 73.92 & 73.70 & 72.64 & 73.17 & 73.60 & 73.30 & 73.71 \\
\hline $\mathrm{Al}_{2} \mathrm{O}_{3}$ & 12.25 & 12.37 & 12.16 & 12.38 & 12.08 & 11.35 & 11.28 & 11.51 & 11.54 & 11.49 & 11.11 & 11.47 & 11.15 & 11.48 \\
\hline $\mathrm{FeO}$ & 1.27 & 1.14 & 1.15 & 1.13 & 1.13 & 0.71 & 0.67 & 0.67 & 0.66 & 0.69 & 0.71 & 0.72 & 0.66 & 0.71 \\
\hline $\mathrm{MgO}$ & 0.11 & 0.09 & 0.12 & 0.11 & 0.10 & 0.05 & 0.06 & 0.03 & 0.04 & 0.05 & 0.05 & 0.03 & 0.06 & 0.03 \\
\hline $\mathrm{CaO}$ & 0.86 & 0.83 & 0.88 & 0.89 & 0.84 & 0.59 & 0.57 & 0.59 & 0.65 & 0.60 & 0.56 & 0.58 & 0.57 & 0.58 \\
\hline $\mathrm{K}_{2} \mathrm{O}$ & 4.19 & 4.06 & 4.10 & 4.09 & 3.99 & 5.19 & 4.93 & 4.97 & 5.02 & 4.91 & 4.71 & 4.65 & 4.87 & 4.94 \\
\hline $\mathrm{Na}_{2} \mathrm{O}$ & 3.60 & 3.68 & 3.46 & 3.78 & 3.76 & 2.88 & 3.03 & 3.00 & 3.08 & 3.24 & 3.09 & 3.20 & 2.96 & 3.15 \\
\hline $\mathrm{TiO}_{2}$ & 0.15 & 0.12 & 0.11 & 0.13 & 0.16 & 0.13 & 0.14 & 0.12 & 0.12 & 0.11 & 0.13 & 0.11 & 0.15 & 0.15 \\
\hline $\mathrm{P}_{2} \mathrm{O}_{5}$ & 0.06 & 0.05 & 0.02 & 0.05 & 0.05 & 0.05 & 0.00 & 0.03 & 0.01 & 0.03 & 0.00 & 0.04 & 0.03 & 0.03 \\
\hline Total & 93.23 & 94.43 & 93.13 & 94.05 & 93.67 & 94.18 & 93.18 & 94.84 & 94.82 & 93.76 & 93.53 & 94.40 & 93.75 & 94.78 \\
\hline $\mathrm{H}_{2} \mathrm{O}$ & 6.77 & 5.57 & 6.87 & 5.95 & 6.33 & 5.82 & 6.82 & 5.16 & 5.18 & 6.24 & 6.47 & 5.60 & 6.25 & 5.22 \\
\hline
\end{tabular}

\begin{tabular}{|c|c|c|c|c|c|c|c|c|c|c|c|c|c|c|}
\hline $\begin{array}{l}\text { Sample } \\
\text { Layer } \\
\text { Shard morphology }\end{array}$ & $\begin{array}{c}758 \mathrm{~B}-4 \mathrm{H}-3, \\
3 \mathrm{~cm} \\
\mathrm{H} \\
\text { Bubble wall }\end{array}$ & $\begin{array}{c}758 \mathrm{~B}-4 \mathrm{H}-3, \\
3 \mathrm{~cm} \\
\mathrm{H} \\
\text { Platy }\end{array}$ & $\begin{array}{c}758 \mathrm{~B}-4 \mathrm{H}-3, \\
3 \mathrm{~cm} \\
\mathrm{H} \\
\text { Bubble wall }\end{array}$ & $\begin{array}{c}758 \mathrm{~B}-4 \mathrm{H}-3 \\
3 \mathrm{~cm} \\
\mathrm{H} \\
?\end{array}$ & $\begin{array}{c}758 \mathrm{~B}-4 \mathrm{H}-3, \\
3 \mathrm{~cm} \\
\mathrm{H} \\
\text { Blocky }\end{array}$ & $\begin{array}{c}758 \mathrm{~B}-4 \mathrm{H}-3 \\
3 \mathrm{~cm} \\
\mathrm{H} \\
?\end{array}$ & $\begin{array}{c}758 \mathrm{~B}-6 \mathrm{H}-2, \\
47 \mathrm{~cm} \\
\mathrm{~h} \\
\text { Blocky }\end{array}$ & $\begin{array}{c}758 \mathrm{~B}-6 \mathrm{H}-2, \\
47 \mathrm{~cm} \\
\mathrm{~h} \\
\text { Bubble wall }\end{array}$ & $\begin{array}{c}758 \mathrm{~B}-6 \mathrm{H}-2 \\
47 \mathrm{~cm} \\
\mathrm{~h} \\
\text { Blocky }\end{array}$ & $\begin{array}{c}758 \mathrm{~B}-6 \mathrm{H}-2, \\
47 \mathrm{~cm} \\
\mathrm{~h} \\
\text { Bubble wall }\end{array}$ & $\begin{array}{c}758 \mathrm{~B}-6 \mathrm{H}-2, \\
47 \mathrm{~cm} \\
\mathrm{~h} \\
\text { Bubble wall }\end{array}$ & $\begin{array}{c}758 \mathrm{~B}-6 \mathrm{H}-2 \\
47 \mathrm{~cm} \\
\mathrm{~h} \\
?\end{array}$ & $\begin{array}{c}758 \mathrm{~B}-6 \mathrm{H}-2, \\
47 \mathrm{~cm} \\
\text { h } \\
\text { Bubble wall }\end{array}$ & $\begin{array}{c}758 \mathrm{~B}-6 \mathrm{H}-2, \\
47 \mathrm{~cm} \\
\mathrm{~h} \\
\text { Bubble wall }\end{array}$ \\
\hline $\mathrm{SiO}_{2}$ & 73.50 & 73.73 & 73.04 & 73.39 & 72.31 & 73.02 & 72.38 & 72.84 & 72.51 & 73.33 & 73.50 & 73.77 & 73.72 & 72.94 \\
\hline $\mathrm{Al}_{2} \mathrm{O}_{3}$ & 11.67 & 11.82 & 11.77 & 11.80 & 11.75 & 11.50 & 11.43 & 11.71 & 11.35 & 11.72 & 11.66 & 11.77 & 11.79 & 11.80 \\
\hline $\mathrm{FeO}$ & 0.81 & 0.84 & 0.78 & 0.74 & 0.76 & 0.77 & 0.69 & 0.66 & 0.64 & 0.66 & 0.65 & 0.65 & 0.71 & 0.65 \\
\hline $\mathrm{MgO}$ & 0.00 & 0.00 & 0.00 & 0.01 & 0.01 & 0.03 & 0.01 & 0.02 & 0.01 & 0.01 & 0.00 & 0.02 & 0.02 & 0.01 \\
\hline $\mathrm{CaO}$ & 0.49 & 0.44 & 0.48 & 0.47 & 0.43 & 0.45 & 0.65 & 0.63 & 0.64 & 0.66 & 0.61 & 0.64 & 0.65 & 0.62 \\
\hline $\mathrm{K}_{2} \mathrm{O}$ & 4.33 & 4.37 & 4.44 & 4.90 & 4.31 & 4.67 & 4.44 & 4.78 & 4.84 & 4.73 & 4.66 & 4.83 & 4.86 & 4.63 \\
\hline $\mathrm{Na}_{2} \mathrm{O}$ & 3.37 & 3.31 & 3.32 & 3.38 & 3.59 & 3.35 & 3.33 & 3.25 & 3.12 & 3.28 & 2.87 & 3.27 & 3.32 & 3.30 \\
\hline $\mathrm{TiO}_{2}$ & 0.03 & 0.06 & 0.04 & 0.06 & 0.05 & 0.07 & 0.04 & 0.05 & 0.06 & 0.03 & 0.04 & 0.05 & 0.04 & 0.05 \\
\hline $\mathrm{P}_{2} \mathrm{O}_{5}^{2}$ & 0.04 & 0.02 & 0.01 & 0.06 & 0.02 & 0.04 & 0.02 & 0.02 & 0.00 & 0.02 & 0.02 & 0.03 & 0.01 & 0.04 \\
\hline Total & 94.24 & 94.59 & 93.88 & 94.81 & 93.23 & 93.90 & 92.99 & 93.96 & 93.17 & 94.44 & 94.01 & 95.03 & 95.12 & 94.04 \\
\hline $\mathrm{H}_{2} \mathrm{O}$ & 5.76 & 5.41 & 6.12 & 5.19 & 6.77 & 6.10 & 7.01 & 6.04 & 6.83 & 5.56 & 5.99 & 4.97 & 4.88 & 5.96 \\
\hline
\end{tabular}




\begin{tabular}{|c|c|c|c|c|c|c|c|c|c|c|c|c|c|c|}
\hline $\begin{array}{l}\text { Sample } \\
\text { Layer } \\
\text { Shard morphology }\end{array}$ & $\begin{array}{c}758 \mathrm{~B}-6 \mathrm{H}-7, \\
17 \mathrm{~cm} \\
1 \\
\text { Platy }\end{array}$ & $\begin{array}{c}758 \mathrm{~B}-6 \mathrm{H}-7 \\
17 \mathrm{~cm} \\
\text { I } \\
\text { Platy }\end{array}$ & $\begin{array}{c}758 \mathrm{~B}-6 \mathrm{H}-7, \\
17 \mathrm{~cm} \\
\quad \\
\text { Bubble wall }\end{array}$ & $\begin{array}{c}758 \mathrm{~B}-6 \mathrm{H}-7, \\
17 \mathrm{~cm} \\
1 \\
?\end{array}$ & $\begin{array}{c}758 \mathrm{~B}-6 \mathrm{H}-7 . \\
17 \mathrm{~cm} \\
1 \\
\text { Blocky }\end{array}$ & $\begin{array}{c}758 \mathrm{~B}-6 \mathrm{H}-7, \\
17 \mathrm{~cm} \\
\quad 1 \\
\text { Bubble wall }\end{array}$ & $\begin{array}{c}758 \mathrm{~B}-6 \mathrm{H}-7, \\
17 \mathrm{~cm} \\
1 \\
\text { Platy }\end{array}$ & $\begin{array}{c}758 \mathrm{~B}-6 \mathrm{H}-7 . \\
17 \mathrm{~cm} \\
1 \\
\text { Platy }\end{array}$ & $\begin{array}{c}758 \mathrm{~B}-6 \mathrm{H}-7, \\
17 \mathrm{~cm} \\
\quad 1 \\
\text { Bubble wall }\end{array}$ & $\begin{array}{c}758 \mathrm{~B}-6 \mathrm{H}-7 . \\
17 \mathrm{~cm} \\
1 \\
\text { Blocky }\end{array}$ & $\begin{array}{c}758 \mathrm{~B}-7 \mathrm{H}-6, \\
29 \mathrm{~cm} \\
\mathrm{~K} \\
\text { Bubble wall }\end{array}$ & $\begin{array}{c}758 \mathrm{~B}-7 \mathrm{H}-6, \\
29 \mathrm{~cm} \\
\mathrm{~K} \\
\text { Platy }\end{array}$ & $\begin{array}{l}758 \mathrm{~B}-7 \mathrm{H}-6, \\
29 \mathrm{~cm} \\
\mathrm{~K} \\
\text { Platy }\end{array}$ & $\begin{array}{c}758 \mathrm{~B}-7 \mathrm{H}-6, \\
29 \mathrm{~cm} \\
\mathrm{~K} \\
\text { Bubble wall }\end{array}$ \\
\hline $\mathrm{SiO}_{2}$ & 73.44 & 73.16 & 73.67 & 73.36 & 72.81 & 72.28 & 73.39 & 72.55 & 72.05 & 73.47 & 71.49 & 72.01 & 72.61 & 71.81 \\
\hline $\mathrm{Al}_{2} \mathrm{O}_{3}$ & 12.00 & 11.64 & 11.85 & 12.14 & 11.95 & 12.02 & 12.04 & 11.79 & 11.83 & 11.91 & 11.51 & 11.58 & 11.67 & 11.69 \\
\hline $\mathrm{FeO}$ & 0.63 & 0.83 & 0.59 & 0.60 & 0.86 & 0.62 & 0.60 & 0.71 & 0.86 & 0.58 & 0.97 & 0.89 & 0.91 & 0.96 \\
\hline $\mathrm{MgO}$ & 0.06 & 0.11 & 0.07 & 0.09 & 0.13 & 0.08 & 0.05 & 0.07 & 0.11 & 0.03 & 0.05 & 0.03 & 0.02 & 0.03 \\
\hline $\mathrm{CaO}$ & 0.65 & 1.09 & 0.67 & 0.69 & 1.14 & 0.67 & 0.67 & 0.95 & 1.01 & 0.67 & 0.57 & 0.52 & 0.52 & 0.56 \\
\hline $\mathrm{K}_{2} \mathrm{O}$ & 4.82 & 3.31 & 4.80 & 4.99 & 3.40 & 4.63 & 4.96 & 4.04 & 3.76 & 5.28 & 4.42 & 4.44 & 4.67 & 4.06 \\
\hline $\mathrm{Na}_{2} \mathrm{O}$ & 2.88 & 3.53 & 3.17 & 3.20 & 3.54 & 3.07 & 3.07 & 3.45 & 3.18 & 2.53 & 3.48 & 3.42 & 3.24 & 3.57 \\
\hline $\mathrm{TiO}_{2}$ & 0.09 & 0.13 & 0.08 & 0.06 & 0.11 & 0.06 & 0.06 & 0.07 & 0.09 & 0.08 & 0.09 & 0.05 & 0.06 & 0.09 \\
\hline $\mathrm{P}_{2} \mathrm{O}_{5}$ & 0.01 & 0.02 & 0.04 & 0.06 & 0.03 & 0.04 & 0.01 & 0.04 & 0.01 & 0.01 & 0.03 & 0.02 & 0.01 & 0.02 \\
\hline Total & 94.58 & 93.82 & 94.94 & 95.19 & 93.97 & 93.47 & 94.85 & 93.67 & 92.90 & 94.56 & 92.61 & 92.96 & 93.71 & 92.79 \\
\hline $\mathrm{H}_{2} \mathrm{O}$ & 5.42 & 6.18 & 5.06 & 4.81 & 6.03 & 6.53 & 5.15 & 6.33 & 7.10 & 5.44 & 7.39 & 7.04 & 6.29 & 7.21 \\
\hline
\end{tabular}

\begin{tabular}{|c|c|c|c|c|c|c|c|c|c|c|c|c|c|}
\hline $\begin{array}{l}\text { Sample } \\
\text { Layer } \\
\text { Shard morphology }\end{array}$ & $\begin{array}{c}\text { 758B-7H-6, } \\
29 \mathrm{~cm} \\
\mathrm{~K} \\
\text { Blocky }\end{array}$ & $\begin{array}{c}758 \mathrm{~B}-7 \mathrm{H}-6, \\
29 \mathrm{~cm} \\
\mathrm{~K} \\
\text { Bubble wall }\end{array}$ & $\begin{array}{c}758 \mathrm{~B}-7 \mathrm{H}-6, \\
29 \mathrm{~cm} \\
\mathrm{~K} \\
\text { Blocky }\end{array}$ & $\begin{array}{c}758 \mathrm{~B}-7 \mathrm{H}-6, \\
29 \mathrm{~cm} \\
K \\
?\end{array}$ & $\begin{array}{c}758 \mathrm{~B}-7 \mathrm{H}-6, \\
29 \mathrm{~cm} \\
\mathrm{~K} \\
\text { Blocky }\end{array}$ & $\begin{array}{c}758 \mathrm{~B}-7 \mathrm{H}-6, \\
29 \mathrm{~cm} \\
\mathrm{~K} \\
\text { Bubble wall }\end{array}$ & $\begin{array}{c}758 \mathrm{~B}-7 \mathrm{H}-\mathrm{CC}, \\
14 \mathrm{~cm} \\
\mathrm{~L} \\
\text { Platy }\end{array}$ & $\begin{array}{c}758 \mathrm{~B}-7 \mathrm{H}-\mathrm{CC}, \\
14 \mathrm{~cm} \\
\mathrm{~L} \\
\text { Bubble wall }\end{array}$ & $\begin{array}{c}\text { 758B-7H-CC, } \\
14 \mathrm{~cm} \\
\mathrm{~L} \\
\text { Blocky }\end{array}$ & $\begin{array}{c}758 \mathrm{~B}-7 \mathrm{H}-\mathrm{CC}, \\
14 \mathrm{~cm} \\
\mathrm{~L} \\
\text { Platy }\end{array}$ & $\begin{array}{c}\text { 758B-7H-CC, } \\
14 \mathrm{~cm} \\
\mathrm{~L} \\
\text { Blocky }\end{array}$ & $\begin{array}{c}758 \mathrm{~B}-7 \mathrm{H}-\mathrm{CC}, \\
14 \mathrm{~cm} \\
\mathrm{~L} \\
\text { Bubble wall }\end{array}$ & $\begin{array}{c}758 \mathrm{~B}-7 \mathrm{H} \cdot \mathrm{CC}, \\
14 \mathrm{~cm} \\
\mathrm{~L} \\
\text { Bubble wall }\end{array}$ \\
\hline $\mathrm{SiO}_{2}$ & 72.04 & 72.28 & 72.09 & 71.81 & 71.10 & 71.47 & 67.20 & 68.59 & 68.05 & 68.39 & 67.79 & 68.05 & 67.40 \\
\hline $\mathrm{Al}_{2} \mathrm{O}_{3}$ & 11.78 & 11.79 & 11.38 & 11.59 & 11.65 & 11.54 & 12.41 & 13.06 & 12.47 & 12.79 & 12.50 & 12.75 & 12.83 \\
\hline $\mathrm{FeO}$ & 1.03 & 0.98 & 0.88 & 0.88 & 1.84 & 0.89 & 2.24 & 2.33 & 2.36 & 2.37 & 2.36 & 2.45 & 2.47 \\
\hline $\mathrm{MgO}$ & 0.04 & 0.02 & 0.03 & 0.04 & 0.08 & 0.03 & 0.26 & 0.24 & 0.23 & 0.25 & 0.28 & 0.28 & 0.30 \\
\hline $\mathrm{CaO}$ & 0.57 & 0.61 & 0.48 & 0.49 & 1.21 & 0.53 & 1.46 & 1.36 & 1.36 & 1.52 & 1.42 & 1.34 & 1.37 \\
\hline $\mathrm{K}_{2} \mathrm{O}$ & 4.45 & 4.64 & 4.46 & 4.40 & 2.87 & 5.04 & 4.49 & 4.42 & 4.32 & 4.21 & 4.60 & 4.42 & 4.38 \\
\hline $\mathrm{Na}_{2} \mathrm{O}$ & 3.33 & 3.43 & 3.36 & 3.47 & 3.79 & 3.16 & 3.72 & 3.40 & 3.66 & 3.73 & 3.57 & 3.95 & 4.13 \\
\hline $\mathrm{TiO}_{2}$ & 0.08 & 0.08 & 0.04 & 0.05 & 0.14 & 0.07 & 0.34 & 0.35 & 0.36 & 0.34 & 0.36 & 0.32 & 0.33 \\
\hline $\mathrm{P}_{2} \mathrm{O}_{5}$ & 0.00 & 0.01 & 0.01 & 0.04 & 0.04 & 0.03 & 0.06 & 0.08 & 0.05 & 0.07 & 0.05 & 0.02 & 0.08 \\
\hline Total & 93.32 & 93.84 & 92.73 & 92.77 & 92.72 & 92.76 & 92.18 & 93.83 & 92.86 & 93.67 & 92.93 & 93.58 & 93.29 \\
\hline $\mathrm{H}_{2} \mathrm{O}$ & 6.68 & 6.16 & 7.27 & 7.23 & 7.28 & 7.24 & 7.82 & 6.17 & 7.14 & 6.33 & 7.07 & 6.42 & 6.71 \\
\hline
\end{tabular}

\begin{tabular}{|c|c|c|c|c|c|c|c|c|c|c|c|c|c|c|}
\hline $\begin{array}{l}\text { Sample } \\
\text { Layer } \\
\text { Shard morphology }\end{array}$ & $\begin{array}{c}758 \mathrm{~B}-7 \mathrm{H}-\mathrm{CC}, \\
14 \mathrm{~cm} \\
\mathrm{~L} \\
\text { Platy }\end{array}$ & $\begin{array}{c}758 \mathrm{~B}-8 \mathrm{H}-4 . \\
111 \mathrm{~cm} \\
\mathrm{M} \\
\text { Bubble wall }\end{array}$ & $\begin{array}{c}758 \mathrm{~B}-8 \mathrm{H}-4, \\
111 \mathrm{~cm} \\
\mathrm{M} \\
\text { Bubble wall }\end{array}$ & $\begin{array}{c}758 \mathrm{~B}-8 \mathrm{H}-4 . \\
111 \mathrm{~cm} \\
\text { M } \\
\text { Platy }\end{array}$ & $\begin{array}{c}758 \mathrm{~B}-8 \mathrm{H}-4, \\
111 \mathrm{~cm} \\
\mathrm{M} \\
\text { Blocky }\end{array}$ & $\begin{array}{c}758 \mathrm{~B}-8 \mathrm{H}-4 . \\
111 \mathrm{~cm} \\
\mathrm{M} \\
\text { Blocky }\end{array}$ & $\begin{array}{c}758 \mathrm{~B}-8 \mathrm{H}-4 . \\
111 \mathrm{~cm} \\
\mathrm{M} \\
\text { Blocky }\end{array}$ & $\begin{array}{c}758 \mathrm{~B}-8 \mathrm{H}-4, \\
111 \mathrm{~cm} \\
\mathrm{M} \\
?\end{array}$ & $\begin{array}{c}758 \mathrm{~B}-8 \mathrm{H}-4 . \\
111 \mathrm{~cm} \\
M \\
\text { Bubble wall }\end{array}$ & $\begin{array}{c}758 \mathrm{~B}-8 \mathrm{H}-4 . \\
111 \mathrm{~cm} \\
\mathrm{M} \\
\text { Bubble wall }\end{array}$ & $\begin{array}{c}758 \mathrm{C}-1 \mathrm{H} \cdot 2 \\
14 \mathrm{~cm} \\
\text { A } \\
\text { Blocky }\end{array}$ & $\begin{array}{c}758 \mathrm{C}-1 \mathrm{H}-2, \\
14 \mathrm{~cm} \\
\text { A } \\
\text { Bubble wall }\end{array}$ & $\begin{array}{c}758 \mathrm{C}-1 \mathrm{H}-2, \\
14 \mathrm{~cm} \\
\mathrm{~A} \\
\text { Bubble wall }\end{array}$ & $\begin{array}{c}758 \mathrm{C}-1 \mathrm{H}-2, \\
14 \mathrm{~cm} \\
\text { A } \\
\text { Blocky }\end{array}$ \\
\hline $\mathrm{SiO}_{2}$ & 68.97 & 71.16 & 70.65 & 72.75 & 72.07 & 72.03 & 71.34 & 71.24 & 72.04 & 71.09 & 71.59 & 70.80 & 73.28 & 72.59 \\
\hline $\mathrm{Al}_{2} \mathrm{O}_{3}$ & 12.78 & 11.91 & 11.84 & 11.66 & 11.67 & 11.80 & 11.77 & 11.65 & 11.87 & 11.56 & 11.48 & 11.44 & 11.90 & 11.85 \\
\hline $\mathrm{FeO}$ & 2.42 & 0.82 & 0.91 & 0.78 & 0.74 & 0.73 & 0.77 & 0.70 & 0.74 & 1.08 & 0.75 & 0.78 & 0.85 & 0.82 \\
\hline $\mathrm{MgO}$ & 0.26 & 0.06 & 0.10 & 0.09 & 0.08 & 0.06 & 0.08 & 0.05 & 0.08 & 0.20 & 0.03 & 0.03 & 0.02 & 0.04 \\
\hline $\mathrm{CaO}$ & 1.37 & 0.95 & 0.99 & 0.88 & 0.93 & 0.81 & 0.96 & 1.03 & 0.79 & 1.79 & 0.69 & 0.69 & 0.77 & 0.81 \\
\hline $\mathrm{K}_{2} \mathrm{O}$ & 4.48 & 4.15 & 3.78 & 4.17 & 4.19 & 4.01 & 4.00 & 4.32 & 4.11 & 3.29 & 4.72 & 4.82 & 4.71 & 5.02 \\
\hline $\mathrm{Na}_{2} \mathrm{O}$ & 3.97 & 3.31 & 3.35 & 3.28 & 3.22 & 3.36 & 3.17 & 3.20 & 3.04 & 3.17 & 3.02 & 3.00 & 2.99 & 2.89 \\
\hline $\mathrm{TiO}_{2}$ & 0.36 & 0.08 & 0.08 & 0.05 & 0.06 & 0.06 & 0.07 & 0.07 & 0.07 & 0.17 & 0.10 & 0.04 & 0.09 & 0.05 \\
\hline $\mathrm{P}_{2} \mathrm{O}_{5}$ & 0.05 & 0.03 & 0.04 & 0.05 & 0.06 & 0.04 & 0.02 & 0.05 & 0.03 & 0.02 & 0.03 & 0.05 & 0.02 & 0.02 \\
\hline Total & 94.66 & 92.47 & 91.74 & 93.71 & 93.02 & 92.90 & 92.18 & 92.31 & 92.77 & 92.37 & 92.41 & 91.65 & 94.63 & 94.09 \\
\hline $\mathrm{H}_{2} \mathrm{O}$ & 5.34 & 7.53 & 8.26 & 6.29 & 6.98 & 7.10 & 7.82 & 7.69 & 7.23 & 7.63 & 7.59 & 8.35 & 5.37 & 5.91 \\
\hline
\end{tabular}




\begin{tabular}{|c|c|c|c|c|c|c|c|c|c|c|c|c|}
\hline $\begin{array}{l}\text { Sample } \\
\text { Layer } \\
\text { Shard morphology }\end{array}$ & $\begin{array}{c}758 \mathrm{C}-1 \mathrm{H}-2, \\
14 \mathrm{~cm} \\
\text { A } \\
\text { Platy }\end{array}$ & $\begin{array}{c}758 \mathrm{C}-1 \mathrm{H}-2 \\
14 \mathrm{~cm} \\
\text { A } \\
\text { Bubble wall }\end{array}$ & $\begin{array}{c}758 \mathrm{C}-1 \mathrm{H}-2 \\
14 \mathrm{~cm} \\
\mathrm{~A} \\
\text { Blocky }\end{array}$ & $\begin{array}{c}758 \mathrm{C}-1 \mathrm{H}-2, \\
14 \mathrm{~cm} \\
\mathrm{~A} \\
\text { Bubble wall }\end{array}$ & $\begin{array}{c}758 \mathrm{C}-1 \mathrm{H}-2, \\
14 \mathrm{~cm} \\
\mathrm{~A} \\
\text { Bubble wall }\end{array}$ & $\begin{array}{c}758 \mathrm{C}-1 \mathrm{H}-2 \\
14 \mathrm{~cm} \\
\mathrm{~A} \\
\text { Blocky }\end{array}$ & $\begin{array}{c}758 \mathrm{C}-1 \mathrm{H}-2, \\
14 \mathrm{~cm} \\
\mathrm{~A} \\
\text { Bubble wall }\end{array}$ & $\begin{array}{c}758 \mathrm{C}-1 \mathrm{H}-2 \\
14 \mathrm{~cm} \\
\mathrm{~A} \\
\text { Bubble wall }\end{array}$ & $\begin{array}{c}758 \mathrm{C}-1 \mathrm{H}-2, \\
14 \mathrm{~cm} \\
\mathrm{~A} \\
\text { Blocky }\end{array}$ & $\begin{array}{c}758 \mathrm{C}-1 \mathrm{H}-2, \\
14 \mathrm{~cm} \\
\mathrm{~A} \\
\text { Blocky }\end{array}$ & $\begin{array}{c}758 \mathrm{C}-1 \mathrm{H}-2, \\
14 \mathrm{~cm} \\
\mathrm{~A} \\
\text { Blocky }\end{array}$ & $\begin{array}{c}758 \mathrm{C}-1 \mathrm{H}-2, \\
14 \mathrm{~cm} \\
\text { A } \\
\text { Bubble wall }\end{array}$ \\
\hline $\mathrm{SiO}_{2}$ & 73.31 & 73.39 & 73.07 & 72.51 & 72.73 & 74.19 & 73.15 & 71.96 & 72.56 & 71.99 & 72.28 & 72.16 \\
\hline $\mathrm{Al}_{2} \mathrm{O}_{3}$ & 11.48 & 11.95 & 12.31 & 11.83 & 11.98 & 11.96 & 12.24 & 12.20 & 11.78 & 11.82 & 12.13 & 12.02 \\
\hline $\mathrm{FeO}$ & 0.73 & 0.80 & 0.84 & 0.82 & 0.83 & 0.79 & 0.83 & 0.81 & 0.79 & 0.82 & 0.84 & 0.79 \\
\hline $\mathrm{MgO}$ & 0.05 & 0.06 & 0.05 & 0.04 & 0.06 & 0.02 & 0.04 & 0.05 & 0.03 & 0.05 & 0.02 & 0.04 \\
\hline $\mathrm{CaO}$ & 0.62 & 0.78 & 0.81 & 0.69 & 0.79 & 0.67 & 0.81 & 1.05 & 0.69 & 0.65 & 0.80 & 0.81 \\
\hline $\mathrm{K}_{2} \mathrm{O}$ & 4.90 & 5.09 & 4.76 & 4.83 & 4.83 & 4.87 & 4.92 & 4.38 & 4.93 & 4.71 & 4.86 & 4.87 \\
\hline $\mathrm{Na}_{2} \mathrm{O}$ & 3.24 & 2.84 & 3.01 & 3.07 & 2.97 & 3.19 & 3.00 & 2.91 & 3.04 & 2.98 & 2.99 & 2.96 \\
\hline $\mathrm{TiO}_{2}$ & 0.06 & 0.07 & 0.06 & 0.06 & 0.08 & 0.04 & 0.06 & 0.06 & 0.06 & 0.07 & 0.07 & 0.07 \\
\hline $\mathrm{P}_{2} \mathrm{O}_{5}$ & 0.01 & 0.03 & 0.00 & 0.03 & 0.04 & 0.01 & 0.01 & 0.02 & 0.02 & 0.05 & 0.04 & 0.03 \\
\hline Total & 94.40 & 95.01 & 94.91 & 93.88 & 94.31 & 95.74 & 95.06 & 93.44 & 93.90 & 93.14 & 94.03 & 93.75 \\
\hline $\mathrm{H}_{2} \mathrm{O}$ & 5.60 & 4.99 & 5.09 & 6.12 & 5.69 & 4.26 & 4.94 & 6.56 & 6.10 & 6.86 & 5.97 & 6.25 \\
\hline
\end{tabular}

\begin{tabular}{|c|c|c|c|c|c|c|c|c|c|c|c|c|}
\hline $\begin{array}{l}\text { Sample } \\
\text { Layer } \\
\text { Shard morphology }\end{array}$ & $\begin{array}{c}758 \mathrm{C}-1 \mathrm{H}-2 \\
14 \mathrm{~cm} \\
\mathrm{~A} \\
\text { Bubble wall }\end{array}$ & $\begin{array}{c}758 \mathrm{C}-1 \mathrm{H}-2, \\
14 \mathrm{~cm} \\
\mathrm{~A} \\
\text { Bubble wall }\end{array}$ & $\begin{array}{c}758 \mathrm{C}-1 \mathrm{H}-2 \\
14 \mathrm{~cm} \\
\mathrm{~A} \\
\text { Bubble wall }\end{array}$ & $\begin{array}{c}758 \mathrm{C}-1 \mathrm{H}-2, \\
14 \mathrm{~cm} \\
\mathrm{~A} \\
\text { Bubble wall }\end{array}$ & $\begin{array}{c}758 \mathrm{C}-1 \mathrm{H}-2, \\
14 \mathrm{~cm} \\
\mathrm{~A} \\
\text { Bubble wall }\end{array}$ & $\begin{array}{c}758 \mathrm{C}-1 \mathrm{H}-2, \\
14 \mathrm{~cm} \\
\text { A } \\
\text { Bubble wall }\end{array}$ & $\begin{array}{c}758 \mathrm{C}-1 \mathrm{H}-2, \\
27 \mathrm{~cm} \\
\mathrm{~A} \\
\text { Blocky }\end{array}$ & $\begin{array}{c}758 \mathrm{C}-1 \mathrm{H}-2 \\
27 \mathrm{~cm} \\
\text { A } \\
\text { Platy }\end{array}$ & $\begin{array}{c}758 \mathrm{C}-1 \mathrm{H}-2 \\
27 \mathrm{~cm} \\
\text { A } \\
\text { Blocky }\end{array}$ & $\begin{array}{c}758 \mathrm{C}-1 \mathrm{H}-2 \\
27 \mathrm{~cm} \\
\text { A } \\
\text { Platy }\end{array}$ & $\begin{array}{c}758 \mathrm{C}-1 \mathrm{H}-2 . \\
27 \mathrm{~cm} \\
\text { A } \\
\text { Bubble wall }\end{array}$ & $\begin{array}{c}758 \mathrm{C}-1 \mathrm{H}-2, \\
27 \mathrm{~cm} \\
\text { A } \\
\text { Platy }\end{array}$ \\
\hline $\mathrm{SiO}_{2}$ & 73.58 & 71.11 & 74.35 & 73.29 & 74.74 & 73.84 & 72.89 & 71.29 & 72.52 & 72.64 & 71.77 & 72.20 \\
\hline $\mathrm{Al}_{2} \mathrm{O}_{3}$ & 11.95 & 11.32 & 11.64 & 11.60 & 11.69 & 11.84 & 11.91 & 12.14 & 11.92 & 12.07 & 11.90 & 11.82 \\
\hline $\mathrm{FeO}$ & 0.74 & 0.75 & 0.77 & 0.77 & 0.76 & 0.82 & 0.83 & 0.79 & 0.81 & 0.79 & 0.81 & 0.79 \\
\hline $\mathrm{MgO}$ & 0.16 & 0.08 & 0.05 & 0.03 & 0.03 & 0.06 & 0.05 & 0.05 & 0.06 & 0.04 & 0.03 & 0.05 \\
\hline $\mathrm{CaO}$ & 0.76 & 0.67 & 0.68 & 0.82 & 0.64 & 0.83 & 0.73 & 0.80 & 0.78 & 0.78 & 0.67 & 0.69 \\
\hline $\mathrm{K}_{2} \mathrm{O}$ & 5.00 & 4.65 & 4.89 & 4.77 & 4.75 & 4.96 & 4.47 & 4.60 & 4.74 & 4.63 & 4.48 & 4.88 \\
\hline $\mathrm{Na}_{2} \mathrm{O}$ & 2.87 & 2.93 & 3.16 & 2.91 & 3.22 & 3.11 & 3.35 & 2.75 & 3.09 & 3.18 & 3.45 & 3.09 \\
\hline $\mathrm{TiO}_{2}$ & 0.09 & 0.05 & 0.08 & 0.07 & 0.08 & 0.11 & 0.06 & 0.05 & 0.07 & 0.06 & 0.05 & 0.06 \\
\hline $\mathrm{P}_{2} \mathrm{O}_{5}$ & 0.04 & 0.00 & 0.02 & 0.03 & 0.02 & 0.00 & 0.05 & 0.04 & 0.02 & 0.04 & 0.05 & 0.02 \\
\hline Total & 95.19 & 91.56 & 95.64 & 94.29 & 95.93 & 95.57 & 94.34 & 92.51 & 94.01 & 94.23 & 93.21 & 93.60 \\
\hline $\mathrm{H}_{2} \mathrm{O}$ & 4.81 & 8.44 & 4.36 & 5.71 & 4.07 & 4.43 & 5.66 & 7.49 & 5.99 & 5.77 & 6.79 & 6.40 \\
\hline
\end{tabular}

\begin{tabular}{|c|c|c|c|c|c|}
\hline $\begin{array}{l}\text { Sample } \\
\text { Layer } \\
\text { Shard morphology }\end{array}$ & $\begin{array}{l}758 \mathrm{C}-1 \mathrm{H}-2, \\
27 \mathrm{~cm} \\
\text { A } \\
\text { Blocky }\end{array}$ & $\begin{array}{c}758 \mathrm{C}-1 \mathrm{H}-2, \\
27 \mathrm{~cm} \\
\text { A } \\
\text { Platy }\end{array}$ & $\begin{array}{c}758 \mathrm{C}-1 \mathrm{H}-2 \\
27 \mathrm{~cm} \\
\text { A } \\
\text { Blocky }\end{array}$ & $\begin{array}{c}758 \mathrm{C}-1 \mathrm{H}-2, \\
27 \mathrm{~cm} \\
\mathrm{~A} \\
\text { Bubble wall }\end{array}$ & $\begin{array}{c}758 \mathrm{C}-1 \mathrm{H}-2, \\
27 \mathrm{~cm} \\
\text { A } \\
\text { Bubble wall }\end{array}$ \\
\hline $\mathrm{SiO}_{2}$ & 71.63 & 73.26 & 71.98 & 70.16 & 71.94 \\
\hline $\mathrm{Al}_{2} \mathrm{O}_{3}$ & 11.93 & 11.98 & 11.97 & 11.81 & 11.90 \\
\hline $\mathrm{FeO}$ & 0.86 & 0.80 & 0.83 & 0.94 & 0.79 \\
\hline $\mathrm{MgO}$ & 0.05 & 0.06 & 0.06 & 0.19 & 0.07 \\
\hline $\mathrm{CaO}$ & 0.80 & 0.70 & 0.72 & 0.83 & 0.81 \\
\hline $\mathrm{K}_{2} \mathrm{O}$ & 4.67 & 4.87 & 4.75 & 4.39 & 4.89 \\
\hline $\mathrm{Na}_{2} \mathrm{O}$ & 3.14 & 3.13 & 2.78 & 3.10 & 2.95 \\
\hline $\mathrm{TiO}_{2}$ & 0.08 & 0.04 & 0.09 & 0.08 & 0.05 \\
\hline $\mathrm{P}_{2} \mathrm{O}_{5}$ & 0.02 & 0.02 & 0.00 & 0.03 & 0.05 \\
\hline Total & 93.18 & 94.86 & 93.18 & 91.53 & 93.45 \\
\hline $\mathrm{H}_{2} \mathrm{O}$ & 6.82 & 5.14 & 6.82 & 8.47 & 6.55 \\
\hline
\end{tabular}



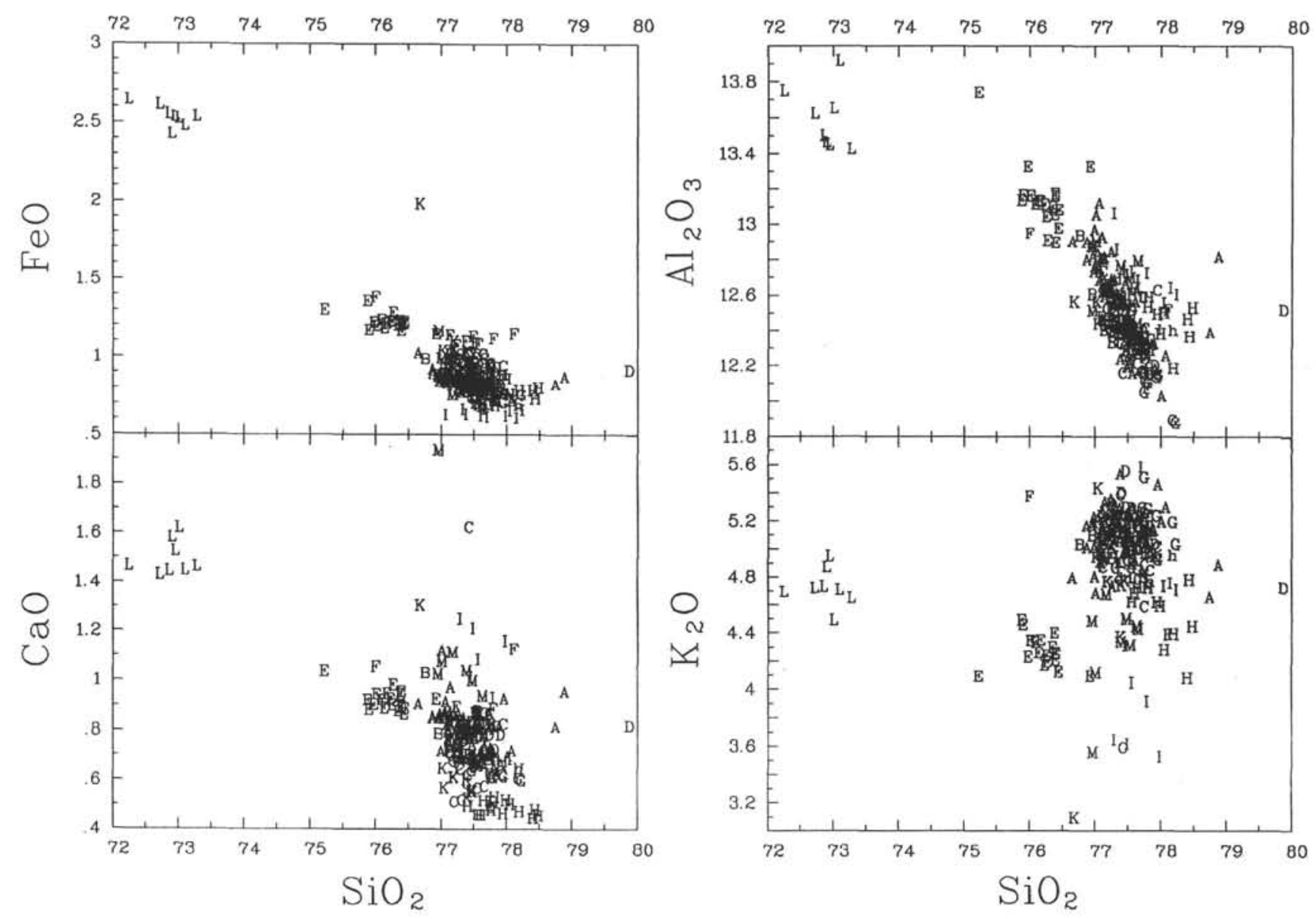

Figure 4. Variation diagrams of $\mathrm{FeO}, \mathrm{CaO}, \mathrm{Al}_{2} \mathrm{O}_{3}$, and $\mathrm{K}_{2} \mathrm{O}$ vs. $\mathrm{SiO}_{2}$. All of the tephra layers are classified as high-silica rhyolites. Layer $\mathrm{L}$ is the least evolved, and layer $\mathrm{A}$ the most evolved. $\mathrm{FeO}, \mathrm{CaO}$, and $\mathrm{Al}_{2} \mathrm{O}_{3}$ display trends resulting from fractionation in the magmas.

This is evident in the $\mathrm{FeO}, \mathrm{CaO}$, and $\mathrm{Al}_{2} \mathrm{O}_{3}$ concentrations in each group.

\section{Feldspar Composition}

Feldspar crystals were analyzed where present in each sample (Tables 3 and 4). A ternary plot of albite, anorthite, and orthoclase shows four distinct groups (Fig. 7). Labradorites and bytownites (An60-90) occur in the least evolved tephras, in layers L, I, and F. The majority of the layers contain andesine with average compositions of An $25-55$ as the main plagioclase phase. Layer I, present in three groups, displays bimodal plagioclase compositions as well as potassium feldspar. Layers $G, D$, and d contain sanidine crystals that plot together around Or69-78. Andesine is also present in these layers. Layer M, containing sodic sanidine crystals, stands alone along the trend, yet separate from the other tephras.

The feldspar compositions correspond to the glass compositions of the tephras (i.e., the least silicic ashes contain plagioclase with the highest anorthite content). The layers containing bytownite and labradorite crystals, L, I, and F, are the least evolved layers in each group defined by the $\mathrm{CaO} / \mathrm{FeO}$ ratio of the glass. Layer $\mathrm{L}$ is the least differentiated of the tephra layers and the oldest in its cogenetic group (Fig. 8). Layer I displays the largest variations in glass and plagioclase compositions. Chesner (1988) described bytownite in the HDT, the least differentiated layer of the Pleistocene Toba tuffs. This layer is correlated through its plagioclase chemistry and eruption time (1.2 Ma) to layer F at Site
758. This indicates, however, that the dacitic whole rock on Sumatra either had a rhyolitic glass matrix or that the uppermost, most differentiated portion of the zoned Toba magma chamber was erupted and subsequently deposited as a marine fallout tephra layer. Chesner (1988) described the zoning in the Toba magma chamber, and the wide range in $\mathrm{CaO}, \mathrm{FeO}, \mathrm{K}_{2} \mathrm{O}$, and $\mathrm{Al}_{2} \mathrm{O}_{3}$ concentrations and plagioclase compositions in layers $\mathrm{I}$ and $\mathrm{M}$ suggests significant zoning within single eruptions.

\section{Other Crystals in the Tephra Layers}

Where present, other crystals in the samples were also analyzed for major oxide composition (Table 5). These crystals were found only in the lowermost part of each tephra layer, which was not always available for sampling. Therefore, the analyses do not represent a statistically valid sample of the entire suite of tephra layers.

Biotite, amphibole, and apatite crystals were measured in layers A, C, d, G, H, I, and K (Fig. 2 and Table 5). The analyses of amphibole crystals in layers A and C closely match those measured in the YTT and the MTT at the Toba caldera on Sumatra (Chesner, 1988).

\section{DATING OF THE TEPHRA LAYERS}

Relative ages were estimated for the ash layers based on their position within the chronostratigraphy provided by the paleomagnetic reversal record and the $\delta^{18} \mathrm{O}$ record (Farrell and Janecek, 


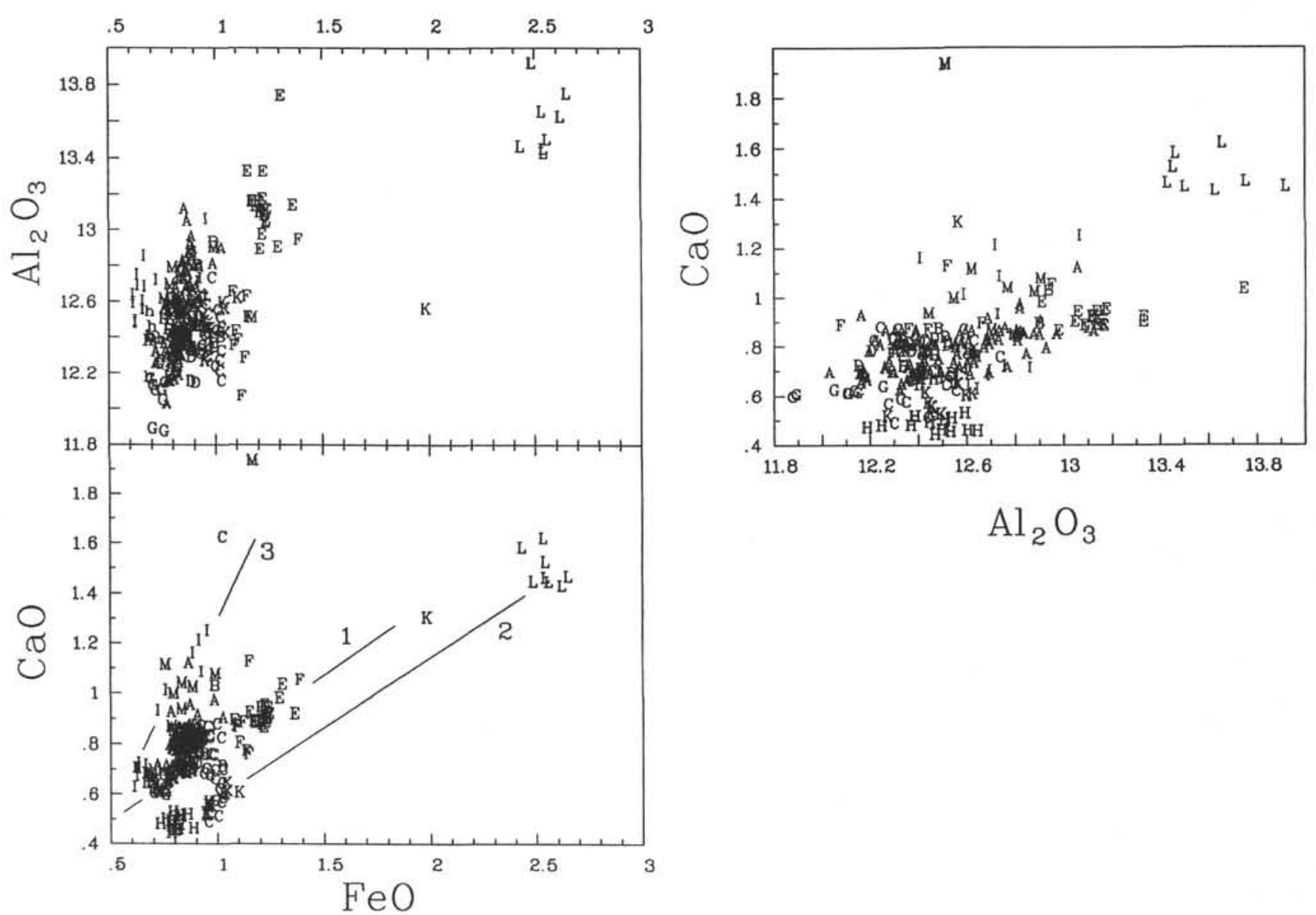

Figure 5. Variation diagrams of $\mathrm{CaO}$ and $\mathrm{Al}_{2} \mathrm{O}_{3}$ vs. $\mathrm{FeO}$ and $\mathrm{CaO}$ vs. $\mathrm{Al}_{2} \mathrm{O}_{3}$. Three groups of layers, best defined for $\mathrm{CaO}$ vs. $\mathrm{FeO}$, represent magmas with similar $\mathrm{CaO} / \mathrm{FeO}$ ratios and thus similar provenance. Group 1 includes layers $\mathrm{A}$ (and $\mathrm{B}$ ), $\mathrm{C}, \mathrm{D}$ (and d), E, F, and G. Group 2 includes layers $\mathrm{H}, \mathrm{K}$, and $\mathrm{L}$; group 3, I and $\mathrm{M}$.

this volume). Paleomagnetic and $\delta^{18} \mathrm{O}$ ages for the ashes were estimated by linearly interpolating between the nearest magnetic reversals and the nearest isotopic control points.

The $\delta^{18} \mathrm{O}$ record from Site 758 , which spans the past $2.5 \mathrm{Ma}$ at an average resolution of $6000 \mathrm{yr}$, provides an independent chronostratigraphy of much higher resolution than the paleomagnetic chronostratigraphy. A preliminary $\delta^{18} \mathrm{O}$-based age model for the past $1.0 \mathrm{Ma}$ was constructed by simple visual correlation of the Site 758 composite $\mathrm{d}^{18} \mathrm{O}$ record to the global average oxygen isotope record, the Spectral Mapping (SPECMAP) stack (Imbrie et al., 1984; Prell et al., 1986), and the Atlantic Site 607 benthic $\delta^{18} \mathrm{O}$ record (Ruddiman et al., 1989; Raymo et al., 1989). The correlations are discussed in detail in Farrell and Janecek (this volume). Initial correlation attempts appear successful, and support for these correlations is provided by comparison to the planktonic and benthic $\delta^{18} \mathrm{O}$ stratigraphy from equatorial Pacific Site 677 (Shackleton and Hall, 1989). Nevertheless, identification of the $\mathrm{d}^{18} \mathrm{O}$ chronostratigraphy in the planktonic foraminifer record from Site 758 is subject to minor revision based on the addition of new benthic foraminifer $\delta^{18} \mathrm{O}$ data.

Table 6 contains a list of what are interpreted to be unique tephra layers representing single eruptions and their age dates. Though the dates acquired by different methods do not exactly match, the correlation between the paleomagnetic dates and $\delta^{18} \mathrm{O}$ dates is good. The differences are primarily due to the lower resolution of the paleomagnetic record.
Ash layer A falls near $\delta^{18} \mathrm{O}$ stage 5.0 (Imbrie et al., 1984), the transition between glacial stage 4 and interglacial stage 5 . The $\delta^{18} \mathrm{O}$ age for layer $\mathrm{A}$ is $0.075 \pm 0.004 \mathrm{Ma}$. This age matches the previously established value of $75,000 \mathrm{yr}$ for the Toba ash (Ninkovich et al., 1978, 1979; Rose and Chesner, 1987, 1988). The age of layer B cannot be differentiated from layer A by paleomagnetic or $\delta^{18} \mathrm{O}$ means, further supporting the conclusion that it is a misplaced part of layer $\mathrm{A}$.

Ash layer $\mathrm{C}$ lies between $\delta^{18} \mathrm{O}$ stages 13.2 and 14.2, which are assigned ages of 0.513 and $0.538 \mathrm{Ma}$, respectively (Imbrie et al., 1984). These dates are close to the date of $0.49 \mathrm{Ma}$ given by Chesner (1988) for the MTT. The age of the layer coupled with the similar chemical compositions strongly supports the correlation of these layers.

Layer D falls just below the paleomagnetic boundary separating the Brunhes and Matuyama Chrons. In the $\delta^{18} \mathrm{O}$ stratigraphy, the ash layer lies between stages $19.2(0.731 \mathrm{Ma})$ and $20.23(0.750$ $\mathrm{Ma}$ ). Ash layer D does not correlate to any previously described eruption from the Toba caldera.

Ash layer $\mathrm{E}$ occurs in the middle of $\delta^{18} \mathrm{O}$ stage 21 , dated at $0.775 \mathrm{Ma}$. This layer is tentatively correlated with the OTT. The OTT, however, is dated at $0.840 \pm 0.30 \mathrm{Ma}$ (Diehl et al., 1987), at least $37,000 \mathrm{yr}$ older than layer E.

Ash layer $\mathrm{F}$ falls between $\delta^{18} \mathrm{O}$ stages 45 and 46 , which are approximately equivalent to 1.273 and $1.294 \mathrm{Ma}$, respectively. This age correlates well with the HDT, dated at $1.20 \pm 0.16 \mathrm{Ma}$. 

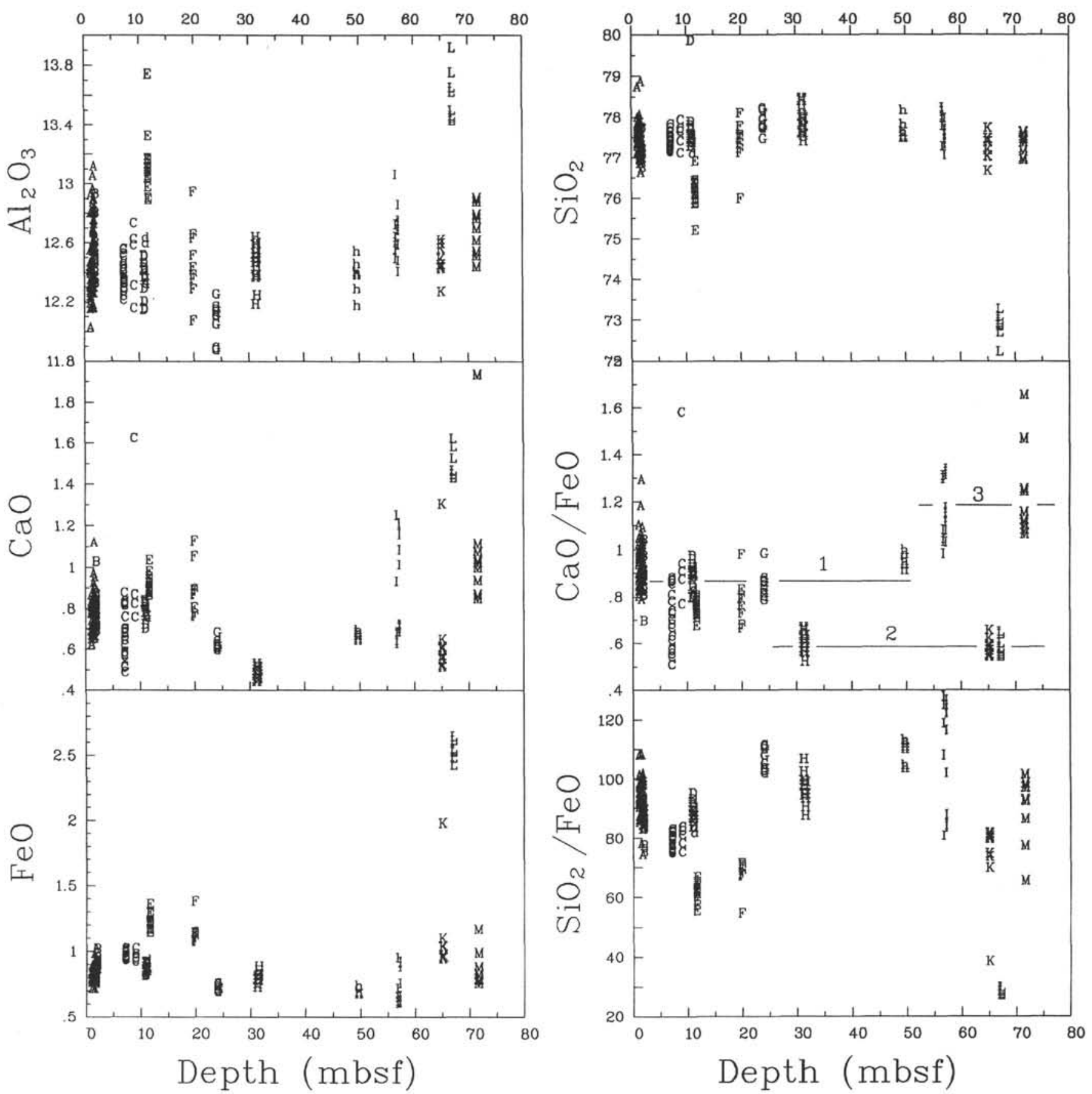

Figure 6. Plots of major oxides and major oxide ratios vs. depth. No clear trend with depth is visible for the entire suite of layers. The three cogenetic groups are visible for $\mathrm{CaO} / \mathrm{FeO}$ vs. depth. Group 2 aligns with a $\mathrm{CaO} / \mathrm{FeO}$ ratio of 0.6 ; group 1 , approximately 0.8 ; and group 3 , approximately 1.2 .

Chemically, layer F is not a dacite; however, it does contain bytownite crystals, as described on land in the HDT.

Layer $\mathrm{G}$ occurs between $\delta^{18} \mathrm{O}$ stages 61 and 63 , in the time interval from 1.596 to $1.636 \mathrm{Ma}$. Ash layer $\mathrm{H}$ lies between stages 92 and 93 , which are from 2.238 to $2.260 \mathrm{Ma}$.

A hiatus of $1.414 \pm 0.016 \mathrm{Ma}$ exists between layers $\mathrm{H}$ and $\mathrm{h}$, the largest gap in the section without deposition of a major fallout tephra layer. The minimum time between major tephra layers is $0.039 \pm 0.010 \mathrm{Ma}$. The average time interval between major tephra layers for the entire sequence is $0.414 \mathrm{Ma}$ with a standard deviation of $0.363 \mathrm{Ma}$. The large standard deviation represents the irregularity of the time intervals between the entire suite of layers.
The ashes are divided into three groups based on their $\mathrm{CaO} / \mathrm{FeO}$ ratios, as defined in Figure 6 (Table 6). The duration of activity producing major tephra layers, or residence time, and the intervals between the eruptions is calculated for each group.

Group 1 (layers A through F) has a residence time of approximately 1.206 Ma. The average time interval between major tephra layers is $0.279 \mathrm{Ma}$ with a standard deviation of 0.208 . Chesner (1988) reported eruption dates of $0.075 \mathrm{Ma}$ for the YTT (layer A), $0.450 \mathrm{Ma}$ for the MTT (layer C), $0.840 \mathrm{Ma}$ for the OTT (layer E), and 1.2 Ma for the HDT (layer F). These data give a mean interval of $0.375 \mathrm{Ma}$ with a standard deviation of 0.015 . 
Group 2 (layers $\mathrm{H}, \mathrm{J}, \mathrm{K}$, and $\mathrm{L}$ ) has a residence time of approximately $2.501 \mathrm{Ma}$. The mean interval between layers is $0.840 \mathrm{Ma}$ with a standard deviation of $1.276 \mathrm{Ma}$. This very high standard deviation is the result of the long hiatus between layers $\mathrm{H}$ and $\mathrm{J}$. Layer J, which was not sampled, is included in this group simply because of its stratigraphic position.

Group 3 (layers I and $\mathrm{M}$ ) has a residence time of approximately $0.935 \mathrm{Ma}$. If layer $\mathrm{h}$ is included in group 3 , the residence time increases to $1.399 \mathrm{Ma}$ with an average interval between major tephras of $0.698 \mathrm{Ma}$ and a standard deviation of $0.334 \mathrm{Ma}$.

\section{DISCUSSION}

\section{Evolution of the Tephras}

The fractionation of quartz, plagioclase, biotite, amphibole, and other mafic minerals, as well as alkali feldspars, is responsible for the chemical difference among the silicic tephra layers. The mafic minerals are generally absent from the tephra layers. Biotite was observed in tephra layers A, C, G, h, and I, and amphibole in layers $\mathrm{A}$ and $\mathrm{K}$. Orthopyroxenes and clinopyroxenes, though not observed in the marine tephra layers, are described by Chesner (1988) in the tuffs and ignimbrites of the Toba caldera. Increasing $\mathrm{FeO}$ and $\mathrm{CaO}$ compositions and decreasing $\mathrm{K}_{2} \mathrm{O}$ concentrations in the highly differentiated layers $\mathrm{A}$ and $\mathrm{H}$ indicate a final crystallization phase of alkali feldspars after the completion of plagioclase and mafic mineral fractionation.

Dacitic crustal melts are inferred as the parent magmas, as required for the genesis of the Toba rhyolites (Chesner, 1988). Magma mixing with, or differentiation from, a basaltic magma is excluded by the large volume of the silicic eruptions $\left(>3000 \mathrm{~km}^{3}\right.$ for Toba alone) and the complete lack of basaltic or andesitic glass shards or lithic clasts.

The bytownite crystals in layers L, I, and F, atypical for rhyolitic ashes, could have resulted as follows.

1. The bytownites are the remainder of a basaltic magma injected into the rhyolite. In this case, sideromelane glass shards and/or resorption or strong zoning of the plagioclase crystals is expected. None of these was observed.

2 . The bytownites crystallized early in the then dacitic magma. This conclusion, however, is contradicted by the high $\mathrm{MgO} / \mathrm{FeO}$ ratio in the bytownites $(>10)$ relative to the magma $(<0.15)$, and is still too high.

3. The magmas could have been contaminated by carbonaterich wall rocks. This excess calcium would then crystallize in the form of the calcium-rich feldspars. The surrounding rocks are Paleozoic metasediments containing carbonate material (Aldiss and Ghazali, 1984; Chesner, 1988) and thus could have been the source for calcium.

4. High initial water pressure may contribute to the crystallization of anorthite-rich plagioclase. Increasing water pressure lowers the crystallization temperature of plagioclase (Deer et al., 1966).

\section{Mechanisms}

The marine fallout tephra layers were formed as a result of highly explosive eruptions, causing the observed high degree of fragmentation and wide dispersal of the glass and crystals. Such eruptions inject ash into the stratosphere, where it can travel for thousands of kilometers before being deposited. The ash is deposited upon the ocean surface, and then settles through the water column. The thickness of the layer and average grain size of the glass shards are directly proportional to the volume and explosivity of the eruption (Shaw et al., 1974).

Because the tectonic movement of Ninetyeast Ridge in the Pliocene-Pleistocene is subparallel to the Indonesian Island Arc
(Peirce, Weissel, et al., 1989), the distance from Sumatra has not significantly changed in the period of marine tephra layer deposition (0-5.1 Ma). Site 758 has moved no more than $3^{\circ}$ northward during this time. Similarly, the water depth of the site has deepened by no more than $150 \mathrm{~m}$ in the last $5 \mathrm{Ma}$ (Shipboard Scientific Party, 1989). Because the thickness of the major ash layers varies from the defined limit of 1 to $34 \mathrm{~cm}$ and the distance from the source has remained virtually constant, then the eruptions have had differing mechanisms or characters. A plot of the $\mathrm{CaO} / \mathrm{FeO}$ ratio vs. the thickness of the ash layer yields trends for the three defined cogenetic groups (Fig. 8). Layers with similar $\mathrm{CaO} / \mathrm{FeO}$ ratios vary in thickness with time. In the case of group 1, layers $\mathrm{F}$ and $\mathrm{E}$, the thinnest layers, are the least evolved and earliest eruptions in the Toba series. As the silica content of the glass increases the thickness of the layers increases and thus the explosivity of the eruptions. This trend is also seen in the two other cogenetic groups.

\section{Time-Related Trends}

From the three defined cogenetic groups, an average residence time of approximately $1.7 \mathrm{Ma}$ can be inferred for these silicic systems. Variations in the residence time are related to the petrogenesis of the magmas. The three defined groups have varying residence times that can be correlated to the range of chemical compositions in the tephra layers. The longest residence time belongs to group 2 , which started with the least differentiated magma. The residence time of the Toba cycle (group 1) of approximately $1.2 \mathrm{Ma}$ is less than that of group 2 (approximately $2.5 \mathrm{Ma}$ ). There is no indication, however, that this cycle is finished. Another eruption in $300,000 \mathrm{yr}$ would extend its residence time, matching it more closely to group 2 .

\section{CONCLUSIONS}

Due to good core recovery and the large number of discrete ash layers, Site 758 provides a unique opportunity to develop a complete tephrochronology. The tephra originated from the Indonesian Island Arc, specifically its northernmost section, Sumatra. All of the tephras are high-silica rhyolites and share similar eruption mechanisms. The ratio of $\mathrm{CaO} / \mathrm{FeO}$ displays three groups of ash layers that may belong to a single magma or magmatic system that has fractionated with time.

The four youngest layers are correlated to the last four eruptions of the Toba caldera in Sumatra. The layers correlate in time as well as chemical composition of the glass shards and feldspars. These layers all belong to the first cogenetic group defined by the $\mathrm{CaO} / \mathrm{FeO}$ ratio. The other two groups have not been correlated to a known source on land.

The thickness of the tephra layers varies, though the distance from the volcanic source has not significantly changed. Thickness of the tephra layers increases with time and silicic character in each group, suggesting that the fractionation of the magmas leads to more explosive eruptions.

The residence time of a given magmatic system is about 1.7 Ma. A less evolved magma has a longer residence time than a more evolved one. This suggests that the composition of the parent magma affects the residence time of the magmatic system.

\section{ACKNOWLEDGMENTS}

We thank Bill Melson, Sorena Sorenson, Gene Jarosewich, Joe Nelen, and Jim Collins of the Smithsonian Institution for useful advice and assistance with the microprobe analyses. This work greatly benefited from the review of Dr. Peter Bitschene and the ODP reviewers Jeffery C. Alt, Craig Chesner, and Bill Rose. Dehn's research was supported by the Deutsche Forschungs Gemeinschaft grant no. Schm 250/37-1 to -3 and the Fulbright 
Table 3. Microprobe analyses of plagioclase crystals in the tephra layers at Site 758.

\begin{tabular}{|c|c|c|c|c|c|c|c|c|c|c|c|c|c|c|}
\hline $\begin{array}{l}\text { Sample } \\
\text { Layer }\end{array}$ & $\begin{array}{c}758 \mathrm{~A}-1 \mathrm{H}-2 \\
25 \mathrm{~cm} \\
\mathrm{~A}\end{array}$ & $\begin{array}{c}758 \mathrm{~A}-1 \mathrm{H}-2 \\
25 \mathrm{~cm} \\
\mathrm{~A}\end{array}$ & $\begin{array}{c}758 \mathrm{~A}-1 \mathrm{H}-2 \\
25 \mathrm{~cm} \\
\mathrm{~A}\end{array}$ & $\begin{array}{c}758 \mathrm{~A}-1 \mathrm{H}-2 \\
25 \mathrm{~cm} \\
\mathrm{~A}\end{array}$ & $\begin{array}{c}758 \mathrm{~A}-1 \mathrm{H}-2 \\
25 \mathrm{~cm} \\
\mathrm{~A}\end{array}$ & $\begin{array}{c}758 \mathrm{~B}-1 \mathrm{H}-1, \\
114 \mathrm{~cm} \\
\mathrm{~A}\end{array}$ & $\begin{array}{c}758 \mathrm{~B}-1 \mathrm{H}-1 \\
114 \mathrm{~cm} \\
\mathrm{~A}\end{array}$ & $\begin{array}{c}758 \mathrm{~B}-1 \mathrm{H}-1 \\
114 \mathrm{~cm} \\
\mathrm{~A}\end{array}$ & $\begin{array}{c}758 \mathrm{~B}-1 \mathrm{H}-1 \\
114 \mathrm{~cm} \\
\mathrm{~A}\end{array}$ & $\begin{array}{c}758 \mathrm{~B}-1 \mathrm{H}-1, \\
114 \mathrm{~cm} \\
\mathrm{~A}\end{array}$ & $\begin{array}{c}758 \mathrm{~B}-1 \mathrm{H}-1 \\
114 \mathrm{~cm} \\
\mathrm{~A}\end{array}$ & $\begin{array}{c}758 \mathrm{~B}-1 \mathrm{H}-1 \\
114 \mathrm{~cm} \\
\mathrm{~A}\end{array}$ & $\begin{array}{c}758 \mathrm{C}-1 \mathrm{H}-2 \\
14 \mathrm{~cm} \\
\mathrm{~A}\end{array}$ & $\begin{array}{c}758 \mathrm{C}-1 \mathrm{H}-2 \\
14 \mathrm{~cm} \\
\mathrm{~A}\end{array}$ \\
\hline $\mathrm{SiO}_{2}$ & 57.67 & 57.24 & 57.42 & 57.51 & 58.60 & 58.94 & 57.38 & 60.35 & 58.76 & 59.34 & 55.09 & 59.69 & 59.36 & 58.33 \\
\hline $\mathrm{TiO}_{2}$ & 0.02 & 0.04 & 0.04 & 0.03 & 0.05 & 0.03 & 0.04 & 0.03 & 0.03 & 0.03 & 0.02 & 0.02 & 0.01 & 0.04 \\
\hline $\mathrm{Al}_{2} \mathrm{O}_{3}$ & 25.83 & 26.12 & 25.84 & 26.20 & 24.83 & 25.65 & 26.40 & 24.68 & 25.59 & 25.00 & 28.24 & 25.17 & 25.14 & 25.87 \\
\hline $\mathrm{Fe}_{2} \mathrm{O}_{3}$ & 0.21 & 0.24 & 0.21 & 0.21 & 0.34 & 0.00 & 0.07 & 0.19 & 0.20 & 0.29 & 0.28 & 0.22 & 0.19 & 0.27 \\
\hline $\mathrm{FeO}$ & 0.00 & 0.00 & 0.00 & 0.00 & 0.00 & 0.19 & 0.11 & 0.00 & 0.00 & 0.00 & 0.00 & 0.00 & 0.00 & 0.00 \\
\hline $\mathrm{MgO}$ & 0.01 & 0.01 & 0.02 & 0.01 & 0.08 & 0.02 & 0.00 & 0.01 & 0.02 & 0.09 & 0.03 & 0.00 & 0.00 & 0.00 \\
\hline $\mathrm{CaO}$ & 8.07 & 8.25 & 7.82 & 7.96 & 6.86 & 7.66 & 8.68 & 6.64 & 7.03 & 6.67 & 10.40 & 6.94 & 6.63 & 7.51 \\
\hline $\mathrm{Na}_{2} \mathrm{O}$ & 6.71 & 6.76 & 6.95 & 6.82 & 7.13 & 6.84 & 6.36 & 7.60 & 7.32 & 7.74 & 5.55 & 7.49 & 7.83 & 7.22 \\
\hline $\mathrm{K}_{2} \mathrm{O}$ & 0.55 & 0.54 & 0.57 & 0.52 & 0.87 & 0.60 & 0.49 & 0.75 & 0.76 & 0.81 & 0.37 & 0.68 & 0.66 & 0.65 \\
\hline $\mathrm{P}_{2}^{2} \mathrm{O}_{5}$ & 0.04 & 0.05 & 0.05 & 0.04 & 0.04 & 0.03 & 0.05 & 0.02 & 0.03 & 0.09 & 0.05 & 0.01 & 0.03 & 0.02 \\
\hline Total & 99.11 & 99.25 & 98.92 & 99.30 & 98.80 & 99.96 & 99.58 & 100.27 & 99.74 & 100.06 & 100.03 & 100.22 & 99.85 & 99.91 \\
\hline $\mathrm{Si}$ & 10.42 & 10.32 & 10.37 & 10.36 & 10.60 & 10.56 & 10.34 & 10.73 & 10.51 & 10.55 & 9.92 & 10.62 & 10.57 & 10.42 \\
\hline $\mathrm{Ti}$ & 0.00 & 0.01 & 0.01 & 0.00 & 0.01 & 0.00 & 0.01 & 0.00 & 0.00 & 0.00 & 0.00 & 0.00 & 0.00 & 0.01 \\
\hline $\mathrm{Al}$ & 5.50 & 5.55 & 5.50 & 5.56 & 5.29 & 5.42 & 5.61 & 5.17 & 5.39 & 5.24 & 5.99 & 5.28 & 5.28 & 5.45 \\
\hline $\mathrm{Fe}^{3+}$ & 0.03 & 0.03 & 0.03 & 0.03 & 0.05 & 0.00 & 0.01 & 0.03 & 0.03 & 0.04 & 0.04 & 0.03 & 0.03 & 0.04 \\
\hline $\mathrm{Fe}^{2+}$ & 0.00 & 0.00 & 0.00 & 0.00 & 0.00 & 0.03 & 0.02 & 0.00 & 0.00 & 0.00 & 0.00 & 0.00 & 0.00 & 0.00 \\
\hline $\mathrm{Mg}$ & 0.00 & 0.00 & 0.01 & 0.00 & 0.02 & 0.01 & 0.00 & 0.00 & 0.01 & 0.02 & 0.01 & 0.00 & 0.00 & 0.00 \\
\hline $\mathrm{Ca}$ & 1.56 & 1.59 & 1.51 & 1.54 & 1.33 & 1.47 & 1.68 & 1.27 & 1.35 & 1.27 & 2.01 & 1.32 & 1.27 & 1.44 \\
\hline $\mathrm{Na}$ & 2.35 & 2.36 & 2.43 & 2.38 & 2.50 & 2.38 & 2.22 & 2.62 & 2.54 & 2.67 & 1.94 & 2.58 & 2.70 & 2.50 \\
\hline $\mathrm{K}$ & 0.13 & 0.12 & 0.13 & 0.12 & 0.20 & 0.14 & 0.11 & 0.17 & 0.17 & 0.18 & 0.08 & 0.15 & 0.15 & 0.15 \\
\hline $\mathbf{P}$ & 0.01 & 0.01 & 0.01 & 0.01 & 0.01 & 0.00 & 0.01 & 0.00 & 0.00 & 0.01 & 0.01 & 0.00 & 0.00 & 0.00 \\
\hline $\operatorname{Sum} Z$ & $\begin{array}{r}15.92 \\
4.04\end{array}$ & $\begin{array}{r}15.87 \\
4.08\end{array}$ & $\begin{array}{r}15.87 \\
4.08\end{array}$ & $\begin{array}{r}15.92 \\
4.04\end{array}$ & $\begin{array}{r}15.89 \\
4.03\end{array}$ & $\begin{array}{r}15.97 \\
3.98\end{array}$ & $\begin{array}{r}15.95 \\
4.01\end{array}$ & $\begin{array}{r}15.91 \\
4.06\end{array}$ & $\begin{array}{r}15.90 \\
4.06\end{array}$ & $\begin{array}{r}15.80 \\
4.12\end{array}$ & $\begin{array}{r}15.91 \\
4.03\end{array}$ & $\begin{array}{r}15.90 \\
4.06\end{array}$ & $\begin{array}{r}15.85 \\
4.12\end{array}$ & $\begin{array}{r}15.87 \\
4.09\end{array}$ \\
\hline Orthoclase & 3.14 & 3.04 & 3.22 & 2.96 & 4.98 & 3.44 & 2.81 & 4.20 & 4.27 & 4.46 & 2.11 & 3.80 & 3.64 & 3.62 \\
\hline Albite & 58.19 & 57.91 & 59.68 & 58.99 & 62.04 & 59.65 & 55.41 & 64.61 & 62.54 & 64.72 & 48.09 & 63.62 & 65.64 & 61.20 \\
\hline Anorthite & 38.67 & 39.05 & 37.10 & 38.05 & 32.98 & 36.91 & 41.79 & 31.19 & 33.19 & 30.82 & 49.80 & 32.58 & 30.72 & 35.18 \\
\hline Sample & $\begin{array}{c}758 \mathrm{C}-1 \mathrm{H}-2 \\
14 \mathrm{~cm} \\
\mathrm{~A}\end{array}$ & $\begin{array}{c}758 \mathrm{C}-1 \mathrm{H}-2 \\
14 \mathrm{~cm} \\
\mathrm{~A}\end{array}$ & $\begin{array}{c}758 \mathrm{C}-1 \mathrm{H}-2 \\
14 \mathrm{~cm} \\
\mathrm{~A}\end{array}$ & $\begin{array}{c}758 \mathrm{C}-1 \mathrm{H}-2 \\
14 \mathrm{~cm} \\
\text { A }\end{array}$ & $\begin{array}{c}758 \mathrm{C}-1 \mathrm{H}-2 \\
27 \mathrm{~cm} \\
\mathrm{~A}\end{array}$ & $\begin{array}{c}758 \mathrm{C}-1 \mathrm{H}-2 \\
27 \mathrm{~cm} \\
\mathrm{~A}\end{array}$ & $\begin{array}{c}758 \mathrm{C}-1 \mathrm{H}-2 \\
27 \mathrm{~cm} \\
\mathrm{~A}\end{array}$ & $\begin{array}{c}758 \mathrm{C}-1 \mathrm{H}-2 \\
27 \mathrm{~cm} \\
\mathrm{~A}\end{array}$ & $\begin{array}{c}758 \mathrm{C}-1 \mathrm{H}-2 \\
27 \mathrm{~cm} \\
\mathrm{~A}\end{array}$ & $\begin{array}{c}758 \mathrm{C}-1 \mathrm{H}-2 \\
27 \mathrm{~cm} \\
\mathrm{~A}\end{array}$ & $\begin{array}{c}758 \mathrm{C}-1 \mathrm{H}-2 \\
27 \mathrm{~cm} \\
\mathrm{~A}\end{array}$ & $\begin{array}{c}758 \mathrm{C}-1 \mathrm{H}-2 \\
27 \mathrm{~cm} \\
\mathrm{~A}\end{array}$ & $\begin{array}{c}758 \mathrm{C}-1 \mathrm{H}-2 \\
27 \mathrm{~cm} \\
\mathrm{~A}\end{array}$ & $\begin{array}{c}758 \mathrm{C}-1 \mathrm{H}-2 \\
27 \mathrm{~cm} \\
\mathrm{~A}\end{array}$ \\
\hline $\mathrm{SiO}_{2}$ & 60.05 & 58.98 & 60.97 & 61.49 & 57.80 & 57.75 & 59.80 & 58.80 & 58.01 & 57.02 & 58.01 & 58.36 & 57.33 & 57.39 \\
\hline $\mathrm{TiO}_{2}^{2}$ & 0.04 & 0.03 & 0.02 & 0.02 & 0.02 & 0.02 & 0.01 & 0.04 & 0.03 & 0.01 & 0.01 & 0.01 & 0.02 & 0.02 \\
\hline $\mathrm{Al}_{2} \mathrm{O}_{3}$ & 24.90 & 25.25 & 24.76 & 24.65 & 26.52 & 26.83 & 24.67 & 24.94 & 25.82 & 26.65 & 25.73 & 25.50 & 25.76 & 25.62 \\
\hline $\mathrm{Fe}_{2} \mathrm{O}_{3}$ & 0.20 & 0.20 & 0.19 & 0.00 & 0.21 & 0.18 & 0.19 & 0.19 & 0.19 & 0.19 & 0.21 & 0.20 & 0.14 & 0.18 \\
\hline $\mathrm{FeO}$ & 0.00 & 0.00 & 0.00 & 0.16 & 0.00 & 0.00 & 0.00 & 0.00 & 0.00 & 0.00 & 0.00 & 0.02 & 0.07 & 0.00 \\
\hline $\mathrm{MgO}$ & 0.00 & 0.00 & 0.00 & 0.00 & 0.00 & 0.01 & 0.00 & 0.00 & 0.01 & 0.00 & 0.00 & 0.00 & 0.01 & 0.00 \\
\hline $\mathrm{CaO}$ & 6.66 & 7.17 & 6.39 & 6.35 & 8.23 & 8.51 & 6.42 & 6.53 & 7.69 & 8.15 & 7.34 & 7.33 & 7.64 & 7.84 \\
\hline $\mathrm{Na}_{2} \mathrm{O}$ & 7.79 & 7.44 & 7.63 & 7.60 & 6.79 & 6.53 & 7.52 & 7.46 & 6.87 & 6.61 & 6.96 & 6.85 & 6.61 & 6.66 \\
\hline $\mathrm{K}_{2} \mathrm{O}$ & 0.69 & 0.73 & 0.84 & 0.91 & 0.54 & 0.51 & 0.79 & 0.83 & 0.65 & 0.56 & 0.72 & 0.73 & 0.63 & 0.65 \\
\hline $\mathrm{P}_{2}^{2} \mathrm{O}_{5}$ & 0.02 & 0.02 & 0.04 & 0.03 & 0.04 & 0.03 & 0.02 & 0.03 & 0.03 & 0.06 & 0.05 & 0.04 & 0.06 & 0.04 \\
\hline Total & 100.35 & 99.82 & 100.84 & 101.21 & 100.15 & 100.37 & 99.42 & 98.82 & 99.30 & 99.25 & 99.03 & 99.04 & 98.27 & 98.40 \\
\hline $\mathrm{Si}$ & 10.66 & 10.54 & 10.79 & 10.84 & 10.33 & 10.31 & 10.73 & 10.60 & 10.45 & 10.28 & 10.47 & 10.54 & 10.45 & 10.44 \\
\hline $\mathrm{Ti}$ & 0.01 & 0.00 & 0.00 & 0.00 & 0.00 & 0.00 & 0.00 & 0.01 & 0.00 & 0.00 & 0.00 & 0.00 & 0.00 & 0.00 \\
\hline Al & 5.21 & 5.32 & 5.16 & 5.12 & 5.58 & 5.65 & 5.21 & 5.30 & 5.48 & 5.66 & 5.47 & 5.43 & 5.53 & 5.49 \\
\hline $\mathrm{Fe}^{3+}$ & 0.03 & 0.03 & 0.03 & 0.00 & 0.03 & 0.02 & 0.03 & 0.03 & 0.03 & 0.03 & 0.03 & 0.03 & 0.02 & 0.02 \\
\hline $\mathrm{Fe}^{2+}$ & 0.00 & 0.00 & 0.00 & 0.02 & 0.00 & 0.00 & 0.00 & 0.00 & 0.00 & 0.00 & 0.00 & 0.00 & 0.01 & 0.00 \\
\hline $\mathrm{K}$ & 0.16 & 0.17 & 0.19 & 0.20 & 0.12 & 0.12 & 0.18 & 0.19 & 0.15 & 0.13 & 0.17 & 0.17 & 0.15 & 0.15 \\
\hline $\mathbf{P}$ & 0.00 & 0.00 & 0.01 & 0.00 & 0.01 & 0.00 & 0.00 & 0.00 & 0.00 & 0.01 & 0.01 & 0.01 & 0.01 & 0.01 \\
\hline Sun $\mathrm{Z}$ & 15.86 & 15.85 & 15.95 & 15.97 & 15.91 & 15.96 & 15.94 & 15.90 & 15.93 & 15.95 & 15.94 & 15.98 & 15.98 & 15.94 \\
\hline Sum $\mathrm{X}$ & 4.10 & 4.12 & 4.02 & 4.00 & 4.05 & 4.01 & 4.03 & 4.06 & 4.03 & 4.02 & 4.02 & 3.99 & 3.97 & 4.03 \\
\hline Orthoclase & 3.81 & 4.04 & 4.72 & 5.11 & 3.04 & 2.90 & 4.49 & 4.70 & 3.70 & $3.2 \mathrm{i}$ & 4.12 & 4.22 & 3.69 & 3.74 \\
\hline Albite & 65.33 & 62.61 & 65.14 & 64.91 & 58.07 & 56.45 & 64.90 & 64.23 & 59.50 & 57.57 & 60.58 & 60.19 & 58.77 & 58.32 \\
\hline Anorthite & 30.86 & 33.34 & 30.15 & 29.97 & 38.89 & 40.65 & 30.62 & 31.07 & 36.80 & 39.22 & 35.30 & 35.59 & 37.54 & 37.94 \\
\hline
\end{tabular}




\begin{tabular}{|c|c|c|c|c|c|c|c|c|c|c|c|c|c|c|}
\hline Layer & $\begin{array}{c}758 \mathrm{~A}-1 \mathrm{H}-2 \\
5 \mathrm{~cm} \\
\mathrm{~A}\end{array}$ & $\begin{array}{c}758 \mathrm{~A}-1 \mathrm{H} \cdot 2 \\
47 \mathrm{~cm} \\
\mathrm{~B}\end{array}$ & $\begin{array}{c}758 \mathrm{~A}-1 \mathrm{H}-2 \\
47 \mathrm{~cm} \\
\mathrm{~B}\end{array}$ & $\begin{array}{c}758 \mathrm{~A}-1 \mathrm{H}-2, \\
47 \mathrm{~cm} \\
\mathrm{~B}\end{array}$ & $\begin{array}{c}758 \mathrm{~A}-1 \mathrm{H}-2 \\
47 \mathrm{~cm} \\
\mathrm{~B}\end{array}$ & $\begin{array}{c}758 \mathrm{~A}-1 \mathrm{H}-2 \\
47 \mathrm{~cm} \\
\mathrm{~B}\end{array}$ & $\begin{array}{c}758 \mathrm{~A}-1 \mathrm{H}-2 \\
47 \mathrm{~cm} \\
\mathrm{~B}\end{array}$ & $\begin{array}{c}758 \mathrm{~A}-1 \mathrm{H}-2 \\
47 \mathrm{~cm} \\
\mathrm{~B}\end{array}$ & $\begin{array}{c}758 \mathrm{~A}-1 \mathrm{H}-2 \\
47 \mathrm{~cm} \\
\mathrm{~B}\end{array}$ & $\begin{array}{c}758 \mathrm{~A}-2 \mathrm{H}-4 \\
38 \mathrm{~cm} \\
\text { D }\end{array}$ & $\begin{array}{c}758 \mathrm{~A}-2 \mathrm{H}-4 \\
38 \mathrm{~cm} \\
\text { D }\end{array}$ & $\begin{array}{c}758 \mathrm{~A}-2 \mathrm{H}-4 \\
38 \mathrm{~cm} \\
\text { D }\end{array}$ & $\begin{array}{c}758 \mathrm{~A}-2 \mathrm{H}-4 \\
38 \mathrm{~cm} \\
\text { D }\end{array}$ & $\begin{array}{c}758 \mathrm{~A}-2 \mathrm{H}-4 \\
115 \mathrm{~cm} \\
\mathrm{E}\end{array}$ \\
\hline $\mathrm{SiO}_{2}$ & 57.92 & 60.57 & 59.90 & 58.06 & 56.95 & 58.48 & 60.13 & 60.73 & 61.18 & 61.30 & 61.38 & 59.36 & 59.72 & 58.84 \\
\hline $\mathrm{TiO}_{2}$ & 0.02 & 0.03 & 0.02 & 0.03 & 0.00 & 0.04 & 0.02 & 0.02 & 0.02 & 0.01 & 0.02 & 0.04 & 0.04 & 0.01 \\
\hline $\mathrm{Al}_{2} \mathrm{O}_{3}$ & 26.70 & 24.46 & 24.19 & 26.25 & 26.19 & 25.64 & 25.05 & 23.86 & 23.79 & 24.12 & 23.68 & $\begin{array}{l}25.24 \\
25\end{array}$ & $\begin{array}{l}25.10 \\
\end{array}$ & 25.68 \\
\hline $\mathrm{Fe}_{2} \mathrm{O}_{3}$ & 0.00 & 0.00 & 0.00 & 0.00 & 0.22 & 0.23 & 0.22 & 0.23 & 0.00 & 0.00 & 0.00 & 0.00 & 0.00 & 0.24 \\
\hline $\mathrm{FeO}$ & 0.17 & 0.21 & 0.18 & 0.22 & 0.00 & 0.00 & 0.00 & 0.00 & 0.22 & 0.17 & 0.19 & 0.19 & 0.20 & 0.00 \\
\hline $\mathrm{MgO}$ & 0.00 & 0.00 & 0.01 & 0.00 & $0.00^{\circ}$ & 0.00 & 0.00 & 0.00 & 0.00 & 0.00 & 0.00 & 0.00 & 0.00 & 0.00 \\
\hline $\mathrm{CaO}$ & 8.37 & 6.61 & 6.74 & 8.43 & 8.39 & 7.84 & 6.79 & 6.19 & 5.86 & 6.08 & 5.93 & 7.00 & 7.23 & 7.28 \\
\hline $\mathrm{Na}_{2} \mathrm{O}$ & 6.27 & 7.06 & 6.94 & 6.42 & 6.49 & 6.97 & 7.46 & 7.71 & 7.63 & 7.41 & 7.37 & 6.75 & 6.88 & 7.29 \\
\hline $\mathrm{K}_{2} \mathrm{O}$ & 0.61 & 0.88 & 0.87 & 0.52 & 0.53 & 0.53 & 0.76 & 0.93 & 1.08 & 0.98 & 0.97 & 0.77 & 0.72 & 0.48 \\
\hline $\mathrm{P}_{2}^{2} \mathrm{O}_{5}$ & 0.05 & 0.02 & 0.02 & 0.01 & 0.04 & 0.01 & 0.01 & 0.03 & 0.01 & 0.00 & 0.00 & 0.01 & 0.00 & 0.03 \\
\hline Total & 100.11 & 99.84 & 98.87 & 99.94 & 98.81 & 99.74 & 100.44 & 99.70 & 99.79 & 100.07 & 99.54 & 99.36 & 99.89 & 99.85 \\
\hline $\mathrm{Si}$ & 10.39 & 10.86 & 10.85 & 10.43 & 10.33 & 10.49 & 10.68 & 10.86 & 10.94 & 10.95 & 11.03 & 10.71 & 10.71 & 10.52 \\
\hline $\mathrm{Ti}$ & 0.00 & 0.00 & 0.00 & 0.00 & 0.00 & 0.01 & 0.00 & 0.00 & 0.00 & 0.00 & 0.00 & 0.01 & 0.01 & 0.00 \\
\hline Al & 5.64 & 5.17 & 5.17 & 5.56 & 5.60 & 5.42 & 5.25 & 5.03 & 5.01 & 5.08 & 5.01 & 5.37 & 5.31 & 5.41 \\
\hline $\mathrm{Fe}^{3+}$ & 0.00 & 0.00 & 0.00 & 0.00 & 0.03 & 0.03 & 0.03 & 0.03 & 0.00 & 0.00 & 0.00 & 0.00 & 0.00 & 0.03 \\
\hline $\mathrm{Fe}^{2+}$ & 0.03 & 0.03 & 0.03 & 0.03 & 0.00 & 0.00 & 0.00 & 0.00 & 0.03 & 0.03 & 0.03 & 0.03 & 0.03 & 0.00 \\
\hline $\mathrm{Mg}$ & 0.00 & 0.00 & 0.00 & 0.00 & 0.00 & 0.00 & 0.00 & 0.00 & 0.00 & 0.00 & 0.00 & 0.00 & 0.00 & 0.00 \\
\hline $\mathrm{Ca}$ & 1.61 & 1.27 & 1.31 & 1.62 & 1.63 & 1.51 & 1.29 & 1.19 & 1.12 & 1.16 & 1.14 & 1.35 & 1.39 & 1.39 \\
\hline $\mathrm{Na}$ & 2.18 & 2.46 & 2.44 & 2.24 & 2.28 & 2.42 & 2.57 & 2.67 & 2.64 & 2.57 & 2.57 & 2.36 & 2.39 & 2.53 \\
\hline $\mathrm{K}$ & 0.14 & 0.20 & 0.20 & 0.12 & 0.12 & 0.12 & 0.17 & 0.21 & 0.25 & 0.22 & 0.22 & 0.18 & 0.16 & 0.11 \\
\hline P & 0.01 & 0.00 & 0.00 & 0.00 & 0.01 & 0.00 & 0.00 & 0.00 & 0.00 & 0.00 & 0.00 & 0.00 & 0.00 & 0.00 \\
\hline Sum Z & 16,04 & 16.03 & 16.02 & 15.98 & 15.93 & 15.91 & 15.93 & 15.89 & 15.95 & 16.02 & 16.04 & 16.07 & 16.02 & 15.93 \\
\hline Sum X & 3.93 & 3.93 & 3.95 & 3.98 & 4.04 & 4.05 & 4.04 & 4.07 & 4.01 & 3.95 & 3.93 & 3.89 & 3.95 & 4.03 \\
\hline Orthoclase & 3.55 & 5.13 & 5.09 & 3.00 & 3.04 & 2.99 & 4.27 & 5.21 & 6.14 & 5.65 & 5.66 & 4.55 & 4.17 & 2.72 \\
\hline Albite & 55.50 & 62.52 & 61.76 & 56.21 & 56.56 & 59.82 & 63.69 & 65.66 & 65.90 & 64.92 & 65.31 & 60.68 & 60.62 & 62.69 \\
\hline Anorthite & 40.94 & 32.35 & 33.15 & 40.79 & 40.40 & 37.18 & 32.04 & 29.13 & 27.97 & 29.43 & 29.04 & 34.77 & 35.20 & 34.59 \\
\hline
\end{tabular}

\begin{tabular}{|c|c|c|c|c|c|c|c|c|c|c|c|c|c|c|}
\hline $\begin{array}{l}\text { Sample } \\
\text { Layer }\end{array}$ & $\begin{array}{c}758 \mathrm{~A}-2 \mathrm{H}-4 \\
115 \mathrm{~cm} \\
\mathrm{E}\end{array}$ & $\begin{array}{c}758 \mathrm{~A}-3 \mathrm{H}-3 \\
125 \mathrm{~cm} \\
\mathrm{~F}\end{array}$ & $\begin{array}{c}758 \mathrm{~A}-3 \mathrm{H}-3 \\
125 \mathrm{~cm} \\
\mathrm{~F}\end{array}$ & $\begin{array}{c}758 \mathrm{~A}-3 \mathrm{H}-3 \\
125 \mathrm{~cm} \\
\mathrm{~F}\end{array}$ & $\begin{array}{c}758 \mathrm{~A}-3 \mathrm{H}-3 \\
125 \mathrm{~cm} \\
\mathrm{~F}\end{array}$ & $\begin{array}{c}758 \mathrm{~A}-3 \mathrm{H}-3 \\
125 \mathrm{~cm} \\
\mathrm{~F}\end{array}$ & $\begin{array}{c}758 \mathrm{~A}-3 \mathrm{H}-3 \\
125 \mathrm{~cm} \\
\mathrm{~F}\end{array}$ & $\begin{array}{c}758 \mathrm{~A}-7 \mathrm{H}-2 \\
120 \mathrm{~cm} \\
1\end{array}$ & $\begin{array}{c}758 \mathrm{~A}-7 \mathrm{H}-2 \\
120 \mathrm{~cm} \\
1\end{array}$ & $\begin{array}{c}758 \mathrm{~A}-7 \mathrm{H}-2 \\
120 \mathrm{~cm} \\
\mathrm{I}\end{array}$ & $\begin{array}{c}758 \mathrm{~A}-7 \mathrm{H}-2 \\
120 \mathrm{~cm} \\
\mathrm{I}\end{array}$ & $\begin{array}{c}758 \mathrm{~A}-7 \mathrm{H}-2 \\
120 \mathrm{~cm} \\
1\end{array}$ & $\begin{array}{c}758 \mathrm{~A}-7 \mathrm{H}-2 \\
120 \mathrm{~cm} \\
\mathrm{I}\end{array}$ & $\begin{array}{c}758 \mathrm{~A}-7 \mathrm{H}-2 \\
120 \mathrm{~cm} \\
\mathrm{I}\end{array}$ \\
\hline $\mathrm{SiO}_{2}$ & 58.31 & 49.50 & 48.92 & 50.25 & 50.26 & 51.26 & 51.43 & 48.63 & 47.29 & 61.19 & 60.65 & 60.95 & 61.03 & 61.22 \\
\hline $\mathrm{TiO}_{2}$ & 0.01 & 0.01 & 0.01 & 0.03 & 0.04 & 0.04 & 0.03 & 0.03 & 0.03 & 0.01 & 0.01 & 0.02 & 0.01 & 0.02 \\
\hline $\mathrm{Al}_{2} \mathrm{O}_{3}$ & 25.16 & 31.48 & 31.69 & 31.25 & 30.91 & 29.96 & 30.34 & 32.36 & 33.13 & 24.26 & 24.39 & 23.88 & 23.50 & 24.27 \\
\hline $\mathrm{Fe}_{2} \mathrm{O}_{3}$ & 0.26 & 0.42 & 0.26 & 0.44 & 0.06 & 0.47 & 0.46 & 0.27 & 0.30 & 0.00 & 0.00 & 0.00 & 0.18 & 0.00 \\
\hline $\mathrm{FeO}$ & 0.00 & 0.00 & 0.18 & 0.04 & 0.41 & 0.00 & 0.00 & 0.00 & 0.00 & 0.13 & 0.17 & 0.15 & 0.00 & 0.13 \\
\hline $\mathrm{MgO}$ & 0.01 & 0.01 & 0.00 & 0.02 & 0.00 & 0.00 & 0.02 & 0.00 & 0.00 & 0.00 & 0.00 & 0.00 & 0.00 & 0.00 \\
\hline $\mathrm{CaO}$ & 6.99 & 14.67 & 14.94 & 14.19 & 14.17 & 12.88 & 12.95 & 15.23 & 15.73 & 5.70 & 5.67 & 5.38 & 5.14 & 5.34 \\
\hline $\mathrm{Na}_{2} \mathrm{O}$ & 7.19 & 3.05 & 2.78 & 3.25 & 3.22 & 3.93 & 3.95 & 2.91 & 2.49 & 7.69 & 7.79 & 7.86 & 8.16 & 7.88 \\
\hline $\mathrm{K}_{2} \mathrm{O}$ & 0.48 & 0.18 & 0.16 & 0.26 & 0.26 & 0.34 & 0.32 & 0.11 & 0.06 & 0.75 & 0.69 & 0.88 & 0.79 & 0.83 \\
\hline $\mathrm{P}_{2}^{2} \mathrm{O}_{5}$ & 0.07 & 0.07 & 0.04 & 0.08 & 0.10 & 0.07 & 0.07 & 0.05 & 0.07 & 0.02 & 0.01 & 0.03 & 0.02 & 0.06 \\
\hline Total & 98.48 & 99.39 & 98.98 & 99.80 & 99.43 & 98.95 & 99.57 & 99.59 & 99.10 & 99.75 & 99.38 & 99.15 & 98.83 & 99.75 \\
\hline $\mathrm{Si}$ & 10.58 & 9.09 & 9.04 & 9.19 & 9.23 & 9.42 & 9.39 & 8.91 & 8.72 & 10.94 & 10.87 & $10.94^{\prime}$ & 10.97 & 10.93 \\
\hline $\mathrm{Ti}$ & 0.00 & 0.00 & 0.00 & 0.00 & 0.01 & 0.01 & 0.00 & 0.00 & 0.00 & 0.00 & 0.00 & 0.00 & 0.00 & 0.00 \\
\hline $\mathrm{Al}_{3+}$ & 5.38 & 6.82 & 6.90 & 6.73 & 6.69 & 6.49 & 6.53 & 6.99 & 7.20 & 5.11 & 5.15 & 5.05 & 4.98 & 5.11 \\
\hline $\mathrm{Fe}^{3+}$ & 0.03 & 0.06 & 0.04 & 0.06 & 0.01 & 0.06 & 0.06 & 0.04 & 0.04 & 0.00 & 0.00 & 0.00 & 0.02 & 0.00 \\
\hline $\mathrm{Fe}^{2+}$ & 0.00 & 0.00 & 0.03 & 0.01 & 0.06 & 0.00 & 0.00 & 0.00 & 0.00 & 0.02 & 0.03 & 0.02 & 0.00 & 0.02 \\
\hline $\mathrm{Mg}$ & 0.00 & 0.00 & 0.00 & 0.01 & 0.00 & 0.00 & 0.01 & & 0.00 & 0.00 & 0.00 & 0.00 & 0.00 & 0.00 \\
\hline $\mathrm{Ca}$ & 1.36 & 2.89 & 2.96 & 2.78 & 2.79 & 2.54 & 2.53 & 2.99 & 3.11 & 1.09 & 1.09 & 1.03 & 0.99 & 1.02 \\
\hline $\mathrm{Na}$ & 2.53 & 1.09 & 1.00 & 1.15 & 1.15 & 1.40 & 1.40 & 1.03 & 0.89 & 2.67 & 2.71 & 2.74 & 2.84 & 2.73 \\
\hline K & 0.11 & 0.04 & 0.04 & 0.06 & 0.06 & 0.08 & 0.07 & 0.03 & 0.01 & 0.17 & 0.16 & 0.20 & 0.18 & 0.19 \\
\hline $\mathbf{P}$ & 0.01 & 0.01 & 0.01 & 0.01 & 0.02 & 0.01 & 0.01 & 0.01 & 0.01 & 0.00 & 0.00 & 0.00 & 0.00 & 0.01 \\
\hline $\operatorname{Sum} Z$ & 15.95 & 15.91 & 15.94 & 15.92 & 15.91 & 15.90 & 15.91 & 15.90 & 15.93 & 16.05 & 16.02 & 16.00 & 15.96 & 16.03 \\
\hline Orthoclase & 2.78 & 1.05 & 0.94 & 1.52 & 1.52 & 1.98 & 1.86 & 0.63 & 0.35 & 4.35 & 3.99 & 5.07 & 4.51 & 4.80 \\
\hline Albite & 63.24 & 27.05 & 24.95 & 28.86 & 28.70 & 34.87 & 34.90 & 25.53 & 22.19 & 67.85 & 68.47 & 68.87 & 70.83 & 69.26 \\
\hline Anorthite & 33.98 & 71.90 & 74.10 & 69.62 & 69.78 & 63.15 & 63.24 & 73.84 & 77.46 & 27.79 & 27.54 & 26.05 & 24.66 & 25.94 \\
\hline
\end{tabular}


Table 3 (continued).

\begin{tabular}{|c|c|c|c|c|c|c|c|c|c|c|c|c|c|c|}
\hline $\begin{array}{l}\text { Sample } \\
\text { Layer }\end{array}$ & $\begin{array}{c}758 \mathrm{~A}-7 \mathrm{H}-2 \\
120 \mathrm{~cm} \\
1\end{array}$ & $\begin{array}{c}758 \mathrm{~B}-1 \mathrm{H}-5, \\
119 \mathrm{~cm} \\
\mathrm{C}\end{array}$ & $\begin{array}{c}758 \mathrm{~B}-1 \mathrm{H}-5, \\
119 \mathrm{~cm} \\
\mathrm{C}\end{array}$ & $\begin{array}{c}758 \mathrm{~B}-1 \mathrm{H}-5, \\
119 \mathrm{~cm} \\
\mathrm{C}\end{array}$ & $\begin{array}{c}758 \mathrm{~B}-1 \mathrm{H}-5, \\
119 \mathrm{~cm} \\
\mathrm{C}\end{array}$ & $\begin{array}{c}758 \mathrm{~B}-1 \mathrm{H}-5, \\
119 \mathrm{~cm} \\
\mathrm{C}\end{array}$ & $\begin{array}{c}758 \mathrm{~B}-1 \mathrm{H}-5, \\
119 \mathrm{~cm} \\
\mathrm{C}\end{array}$ & $\begin{array}{c}758 \mathrm{~B}-2 \mathrm{H}-2 \\
25 \mathrm{~cm} \\
\mathrm{~d}\end{array}$ & $\begin{array}{c}758 \mathrm{~B}-2 \mathrm{H}-2, \\
25 \mathrm{~cm} \\
\mathrm{~d}\end{array}$ & $\begin{array}{c}758 \mathrm{~B}-2 \mathrm{H}-2, \\
25 \mathrm{~cm} \\
\mathrm{~d}\end{array}$ & $\begin{array}{c}758 \mathrm{~B}-2 \mathrm{H}-2, \\
25 \mathrm{~cm} \\
\mathrm{~d}\end{array}$ & $\begin{array}{c}758 \mathrm{~B}-2 \mathrm{H}-2 \\
25 \mathrm{~cm} \\
\mathrm{~d}\end{array}$ & $\begin{array}{c}758 \mathrm{~B}-2 \mathrm{H}-2 \\
25 \mathrm{~cm} \\
\mathrm{~d}\end{array}$ & $\begin{array}{c}758 \mathrm{~B}-2 \mathrm{H}-2, \\
61 \mathrm{~cm} \\
\mathrm{E}\end{array}$ \\
\hline $\mathrm{SiO}_{2}$ & 61.85 & 58.03 & 57.82 & 57.88 & 57.10 & 56.54 & 56.19 & 58.96 & 57.35 & 58.09 & 58.25 & 60.19 & 60.38 & 56.73 \\
\hline $\mathrm{TiO}_{2}$ & 0.01 & 0.01 & 0.02 & 0.01 & 0.02 & 0.01 & 0.01 & 0.04 & 0.03 & 0.00 & 0.01 & 0.02 & 0.01 & 0.11 \\
\hline $\mathrm{Al}_{2} \mathrm{O}_{3}$ & 23.47 & 26.20 & 26.11 & 25.95 & 26.57 & 27.21 & 27.23 & 25.92 & 26.42 & 25.52 & 25.79 & 25.14 & 24.98 & 25.73 \\
\hline $\mathrm{Fe}_{2} \mathrm{O}_{3}$ & 0.00 & 0.00 & 0.23 & 0.00 & 0.22 & 0.11 & 0.22 & 0.00 & 0.00 & 0.00 & 0.04 & 0.00 & 0.00 & 0.00 \\
\hline $\mathrm{FeO}$ & 0.11 & 0.19 & 0.00 & 0.21 & 0.00 & 0.09 & 0.00 & 0.21 & 0.17 & 0.19 & 0.13 & 0.18 & 0.19 & 1.06 \\
\hline $\mathrm{MgO}$ & 0.00 & 0.01 & 0.00 & 0.00 & 0.00 & 0.00 & 0.00 & 0.00 & 0.00 & 0.00 & 0.00 & 0.01 & 0.00 & 0.16 \\
\hline $\mathrm{CaO}$ & 4.92 & 7.96 & 8.08 & 7.84 & 8.38 & 8.94 & 9.03 & 7.94 & 8.89 & 7.61 & 7.90 & 7.25 & 7.06 & 9.87 \\
\hline $\mathrm{Na}_{2} \mathrm{O}$ & 8.13 & 6.51 & 6.61 & 6.50 & 6.45 & 6.10 & 6.13 & 6.39 & 5.99 & 6.63 & 6.72 & 6.76 & 6.89 & 5.26 \\
\hline $\mathrm{K}_{2} \mathrm{O}$ & 0.82 & 0.59 & 0.60 & 0.66 & 0.57 & 0.44 & 0.39 & 0.63 & 0.53 & 0.59 & 0.57 & 0.89 & 0.83 & 0.44 \\
\hline $\mathrm{P}_{2} \mathrm{O}_{5}$ & 0.02 & 0.03 & 0.01 & 0.03 & 0.04 & 0.02 & 0.05 & 0.07 & 0.04 & 0.04 & 0.02 & 0.01 & 0.02 & 0.04 \\
\hline Total & 99.33 & 99.53 & 99.48 & 99.08 & 99.35 & 99.46 & 99.25 & 100.16 & 99.42 & 98.67 & 99.43 & 100.45 & 100.36 & 99.40 \\
\hline $\mathrm{Si}$ & 11.07 & 10.45 & 10.42 & 10.47 & 10.30 & 10.21 & 10.16 & 10.58 & 10.38 & 10.55 & 10.49 & 10.75 & 10.78 & 10.35 \\
\hline Ti & 0.00 & 0.00 & 0.00 & 0.00 & 0.00 & 0.00 & 0.00 & 0.01 & 0.00 & 0.00 & 0.00 & 0.00 & 0.00 & 0.02 \\
\hline Al & 4.95 & 5.56 & 5.54 & 5.53 & 5.65 & 5.79 & 5.81 & 5.48 & 5.64 & 5.46 & 5.48 & 5.29 & 5.26 & 5.53 \\
\hline $\mathrm{Fe}^{3+}$ & 0.00 & 0.00 & 0.03 & 0.00 & 0.03 & 0.02 & 0.03 & 0.00 & 0.00 & 0.00 & 0.01 & 0.00 & 0.00 & 0.00 \\
\hline $\mathrm{Fe}^{2+}$ & 0.02 & 0.03 & 0.00 & 0.03 & 0.00 & 0.01 & 0.00 & 0.03 & 0.03 & 0.03 & 0.02 & 0.03 & 0.03 & 0.16 \\
\hline $\mathrm{Mg}$ & 0.00 & 0.00 & 0.00 & 0.00 & 0.00 & 0.00 & 0.00 & 0.00 & 0.00 & 0.00 & 0.00 & 0.00 & 0.00 & 0.04 \\
\hline $\mathrm{Ca}$ & 0.94 & 1.54 & 1.56 & 1.52 & 1.62 & 1.73 & 1.75 & 1.53 & 1.72 & 1.48 & 1.52 & 1.39 & 1.35 & 1.93 \\
\hline $\mathrm{Na}$ & 2.82 & 2.27 & 2.31 & 2.28 & 2.26 & 2.14 & 2.15 & 2.22 & 2.10 & 2.33 & 2.35 & 2.34 & 2.39 & 1.86 \\
\hline $\mathrm{K}$ & 0.19 & 0.14 & 0.14 & 0.15 & 0.13 & 0.10 & 0.09 & 0.14 & 0.12 & 0.14 & 0.13 & 0.20 & 0.19 & 0.10 \\
\hline P & 0.00 & 0.00 & 0.00 & 0.00 & 0.01 & 0.00 & 0.01 & 0.01 & 0.01 & 0.01 & 0.00 & 0.00 & 0.00 & 0.01 \\
\hline Sum Z & 16.03 & 16.02 & 15.96 & 16.01 & 15.95 & 16.00 & 15.97 & 16.06 & 16.02 & 16.01 & 15.97 & 16.04 & 16.04 & 15.88 \\
\hline Sum X & 3.95 & 3.95 & 4.01 & 3.95 & 4.01 & 3.97 & 3.99 & 3.89 & 3.95 & 3.95 & 4.00 & 3.93 & 3.93 & 3.89 \\
\hline Orthoclase & 4.74 & 3.44 & 3.44 & 3.85 & 3.27 & 2.56 & 2.26 & 3.70 & 3.10 & 3.46 & 3.27 & 5.16 & 4.82 & 2.63 \\
\hline Anorthite & 23.87 & 38.94 & 38.93 & 38.45 & 40.42 & 43.60 & 43.86 & 39.20 & 43.66 & 37.47 & 38.09 & 35.29 & 34.41 & 49.57 \\
\hline $\begin{array}{l}\text { Sample } \\
\text { Layer }\end{array}$ & $\begin{array}{c}758 \mathrm{~B}-2 \mathrm{H}-2, \\
61 \mathrm{~cm} \\
\mathrm{E}\end{array}$ & $\begin{array}{c}758 \mathrm{~B}-2 \mathrm{H}-2, \\
61 \mathrm{~cm} \\
\mathrm{E}\end{array}$ & $\begin{array}{c}758 \mathrm{~B}-2 \mathrm{H}-2, \\
61 \mathrm{~cm} \\
\mathrm{E}\end{array}$ & $\begin{array}{c}758 \mathrm{~B}-2 \mathrm{H}-2, \\
61 \mathrm{~cm} \\
\mathrm{E}\end{array}$ & $\begin{array}{c}758 \mathrm{~B}-2 \mathrm{H}-2, \\
61 \mathrm{~cm} \\
\mathrm{E}\end{array}$ & $\begin{array}{c}758 \mathrm{~B}-3 \mathrm{H}-4, \\
69 \mathrm{~cm} \\
\mathrm{G}\end{array}$ & $\begin{array}{c}758 \mathrm{~B}-3 \mathrm{H}-4, \\
69 \mathrm{~cm} \\
\mathrm{G}\end{array}$ & $\begin{array}{c}758 \mathrm{~B}-3 \mathrm{H}-4 \\
69 \mathrm{~cm} \\
\mathrm{G}\end{array}$ & $\begin{array}{c}758 \mathrm{~B}-3 \mathrm{H}-4 \\
69 \mathrm{~cm} \\
\mathrm{G}\end{array}$ & $\begin{array}{c}758 \mathrm{~B}-6 \mathrm{H}-7, \\
17 \mathrm{~cm} \\
1\end{array}$ & $\begin{array}{c}758 \mathrm{~B}-6 \mathrm{H}-7, \\
17 \mathrm{~cm} \\
1\end{array}$ & $\begin{array}{c}758 \mathrm{~B}-6 \mathrm{H}-7, \\
17 \mathrm{~cm} \\
\mathrm{I}\end{array}$ & $\begin{array}{c}758 \mathrm{~B}-6 \mathrm{H}-7, \\
17 \mathrm{~cm} \\
\mathrm{I}\end{array}$ & $\begin{array}{c}758 \mathrm{~B}-7 \mathrm{H}-6, \\
29 \mathrm{~cm} \\
\mathrm{~K}\end{array}$ \\
\hline $\mathrm{SiO}_{2}$ & 55.39 & 60.38 & 60.29 & 55.93 & 56.17 & 60.27 & 60.33 & 61.63 & 60.99 & 59.84 & 60.03 & 58.78 & 59.51 & 55.17 \\
\hline $\mathrm{TiO}_{2}$ & 0.12 & 0.03 & 0.01 & 0.04 & 0.06 & 0.01 & 0.03 & 0.02 & 0.03 & 0.04 & 0.01 & 0.01 & 0.02 & 0.08 \\
\hline $\mathrm{Al}_{2} \mathrm{O}_{3}$ & 27.05 & 24.10 & 24.27 & 26.40 & 26.34 & 23.80 & 24.01 & 22.95 & 23.85 & 24.81 & 24.74 & 25.06 & 24.78 & 25.99 \\
\hline $\mathrm{Fe}_{2} \mathrm{O}_{3}$ & 0.00 & 0.00 & 0.00 & 0.00 & 0.00 & 0.00 & 0.00 & 0.00 & 0.00 & 0.12 & 0.14 & 0.16 & 0.16 & 0.88 \\
\hline $\mathrm{FeO}$ & 0.87 & 0.19 & 0.19 & 0.49 & 0.46 & 0.23 & 0.21 & 0.27 & 0.23 & 0.00 & 0.00 & 0.00 & 0.00 & 0.00 \\
\hline MgO & 0.08 & 0.00 & 0.00 & 0.49 & $\begin{array}{l}0.40 \\
0.02\end{array}$ & 0.00 & 0.00 & 0.00 & 0.00 & 0.00 & 0.01 & 0.01 & 0.02 & 0.11 \\
\hline $\mathrm{CaO}$ & 10.62 & 6.46 & 6.64 & 9.84 & 9.61 & 6.12 & 6.09 & 5.93 & 6.10 & 6.31 & 6.33 & 7.22 & 6.69 & 9.41 \\
\hline $\mathrm{Na}_{2} \mathrm{O}$ & 5.01 & 7.09 & 7.25 & 5.46 & 5.49 & 7.03 & 7.06 & 6.68 & 7.07 & 7.71 & 7.74 & 7.60 & $\begin{array}{l}7.06 \\
7.62\end{array}$ & 5.95 \\
\hline $\mathrm{K}_{2} \mathrm{O}$ & 0.32 & 0.61 & 0.59 & 0.64 & 0.70 & 0.89 & 0.86 & 1.14 & 0.88 & 0.62 & 0.66 & 0.41 & 0.51 & 0.37 \\
\hline $\mathrm{P}_{2} \mathrm{O}_{5}$ & 0.05 & 0.05 & 0.04 & 0.07 & 0.03 & 0.01 & 0.02 & 0.05 & 0.06 & 0.06 & 0.05 & 0.02 & 0.05 & 0.06 \\
\hline Total & 99.51 & 98.91 & 99.28 & 98.88 & 98.88 & 98.36 & 98.61 & 98.67 & 99.21 & 99.51 & 99.71 & 99.27 & 99.36 & 98.02 \\
\hline $\mathrm{Si}$ & 10.09 & 10.93 & 10.86 & 10.22 & 10.26 & 10.97 & 10.95 & 11.23 & 11.02 & 10.71 & 10.72 & 10.55 & 10.67 & 10.14 \\
\hline $\mathrm{Ti}$ & 0.02 & 0.00 & 0.00 & 0.01 & 0.01 & 0.00 & 0.00 & 0.00 & 0.00 & 0.01 & 0.00 & 0.00 & 0.00 & 0.01 \\
\hline $\mathrm{Al}$ & 5.81 & 5.14 & 5.15 & 5.68 & 5.67 & 5.11 & 5.14 & 4.93 & 5.08 & 5.23 & 5.21 & 5.30 & 5.24 & 5.63 \\
\hline $\mathrm{Fe}^{3+}$ & 0.00 & 0.00 & 0.00 & 0.00 & 0.00 & 0.00 & 0.00 & 0.00 & 0.00 & 0.02 & 0.02 & 0.02 & 0.02 & 0.12 \\
\hline $\mathrm{Fe}^{2+}$ & 0.13 & $\begin{array}{l}0.03 \\
0.03\end{array}$ & 0.03 & 0.07 & 0.07 & 0.04 & 0.03 & 0.04 & 0.03 & $\begin{array}{l}0.02 \\
0.00\end{array}$ & $\begin{array}{l}0.02 \\
0.00\end{array}$ & 0.00 & $\begin{array}{l}0.02 \\
0.00\end{array}$ & 0.00 \\
\hline $\mathrm{Mg}$ & 0.02 & 0.00 & 0.00 & 0.00 & 0.01 & 0.00 & 0.00 & 0.00 & 0.00 & 0.00 & 0.00 & 0.00 & 0.01 & 0.03 \\
\hline $\mathrm{Ca}$ & 2.07 & 1.25 & 1.28 & 1.93 & 1.88 & 1.19 & 1.18 & 1.16 & 1.18 & 1.21 & 1.21 & 1.39 & 1.29 & 1.85 \\
\hline $\mathrm{Na}$ & 1.77 & 2.49 & 2.53 & 1.93 & 1.94 & 2.48 & 2.49 & 2.36 & 2.48 & 2.68 & 2.68 & 2.64 & 2.65 & 2.12 \\
\hline $\mathrm{K}$ & 0.07 & 0.14 & 0.14 & 0.15 & 0.16 & 0.21 & 0.20 & 0.27 & 0.20 & 0.14 & 0.15 & 0.09 & 0.12 & 0.09 \\
\hline P & 0.01 & 0.01 & 0.01 & 0.01 & 0.00 & 0.00 & 0.00 & 0.01 & 0.01 & 0.01 & 0.01 & 0.00 & 0.01 & 0.01 \\
\hline Sum Z & 15.90 & 16.08 & 16.01 & 15.90 & 15.92 & 16.08 & 16.09 & 16.16 & 16.09 & 15.94 & 15.93 & 15.85 & 15.91 & 15.77 \\
\hline Sum X & 3.92 & 3.88 & 3.95 & 4.01 & 3.99 & 3.88 & 3.87 & 3.78 & 3.86 & 4.03 & 4.04 & 4.13 & 4.05 & 4.06 \\
\hline Orthoclase & 1.90 & 3.63 & 3.43 & 3.72 & 4.09 & 5.32 & 5.15 & 7.01 & 5.25 & 3.52 & 3.72 & 2.27 & 2.88 & 2.14 \\
\hline Albite & $\begin{array}{r}1.90 \\
45.18\end{array}$ & $\begin{array}{r}5.03 \\
64.10\end{array}$ & $\begin{array}{r}3.43 \\
64.12\end{array}$ & $\begin{array}{r}3.12 \\
48.24\end{array}$ & $\begin{array}{l}4.09 \\
48.75\end{array}$ & $\begin{array}{r}3.32 \\
63.92\end{array}$ & $\begin{array}{r}64.23 \\
64\end{array}$ & 62.39 & 64.16 & 66.44 & 66.31 & $\begin{array}{r}2.27 \\
64.08\end{array}$ & $\begin{array}{r}6.80 \\
65.39\end{array}$ & $\begin{array}{r}52.22 \\
52.22\end{array}$ \\
\hline Anorthite & 52.92 & 32.27 & 32.45 & 48.04 & 47.16 & 30.75 & 30.62 & 30.61 & 30.59 & 30.05 & 29.97 & 33.64 & 31.73 & 45.64 \\
\hline
\end{tabular}




\begin{tabular}{|c|c|c|c|c|c|c|c|c|c|}
\hline $\begin{array}{l}\text { Sample } \\
\text { Layer }\end{array}$ & $\begin{array}{c}7588-7 \mathrm{H}-6, \\
29 \mathrm{~cm} \\
\mathrm{~K}\end{array}$ & $\begin{array}{c}758 \mathrm{~B}-7 \mathrm{H}-6, \\
29 \mathrm{~cm} \\
\mathrm{~K}\end{array}$ & $\begin{array}{c}7588-7 \mathrm{H}-6, \\
29 \mathrm{~cm} \\
\mathrm{~K}\end{array}$ & $\begin{array}{c}758 \mathrm{~B}-7 \mathrm{H}-\mathrm{CC}, \\
14 \mathrm{~cm} \\
\mathrm{~L}\end{array}$ & $\begin{array}{c}758 \mathrm{~B}-7 \mathrm{H}-\mathrm{CC} \\
14 \mathrm{~cm} \\
\mathrm{~L}\end{array}$ & $\begin{array}{c}758 \mathrm{~B}-7 \mathrm{H}-\mathrm{CC} \\
14 \mathrm{~cm} \\
\mathrm{~L}\end{array}$ & $\begin{array}{c}758 \mathrm{~B}-7 \mathrm{H}-\mathrm{CC}, \\
14 \mathrm{~cm} \\
\mathrm{~L}\end{array}$ & $\begin{array}{c}758 \mathrm{~B}-7 \mathrm{H}-\mathrm{CC} \\
14 \mathrm{~cm} \\
\mathrm{~L}\end{array}$ & $\begin{array}{c}758 \mathrm{~B}-7 \mathrm{H}-\mathrm{CC}, \\
14 \mathrm{~cm} \\
\mathrm{~L}\end{array}$ \\
\hline $\mathrm{SiO}_{2}$ & 54.06 & 54.90 & 54.17 & 49.18 & 49.16 & 48.00 & 49.37 & 45.43 & 45.74 \\
\hline $\mathrm{TiO}_{2}^{-}$ & 0.07 & 0.08 & 0.09 & 0.07 & 0.05 & 0.05 & 0.05 & 0.04 & 0.05 \\
\hline $\mathrm{Al}_{2} \mathrm{O}_{3}$ & 27.64 & 27.05 & 27.33 & 31.62 & 30.91 & 31.73 & 30.08 & 33.07 & 32.86 \\
\hline $\mathrm{Fe}_{2} \mathrm{O}_{3}$ & 0.84 & 0.64 & 0.62 & 0.54 & 0.56 & 0.66 & 0.69 & 0.67 & 0.72 \\
\hline $\mathrm{FeO}$ & 0.00 & 0.00 & 0.00 & 0.00 & 0.00 & 0.00 & 0.00 & 0.00 & 0.00 \\
\hline $\mathrm{MgO}$ & 0.08 & 0.06 & 0.09 & 0.06 & 0.03 & 0.06 & 0.09 & 0.09 & 0.09 \\
\hline $\mathrm{CaO}$ & 10.79 & 9.74 & 10.12 & 14.47 & 14.50 & 15.62 & 14.22 & 17.15 & 16.92 \\
\hline $\mathrm{Na}_{2} \mathrm{O}$ & 5.38 & 5.90 & 5.61 & 3.10 & 3.26 & 2.83 & 3.73 & 1.65 & 1.83 \\
\hline $\mathrm{K}_{2} \mathrm{O}$ & 0.31 & 0.46 & 0.43 & 0.13 & 0.10 & 0.04 & 0.09 & 0.04 & 0.04 \\
\hline $\mathrm{P}_{2} \mathrm{O}_{5}$ & 0.06 & 0.06 & 0.09 & 0.10 & 0.10 & 0.08 & 0.08 & 0.10 & 0.12 \\
\hline Total & 99.23 & 98.89 & 98.55 & 99.27 & 98.67 & 99.07 & 98.40 & 98.24 & 98.37 \\
\hline $\mathrm{Si}$ & 9.84 & 9.99 & 9.90 & 9.04 & 9.09 & 8.86 & 9.13 & 8.51 & 8.55 \\
\hline $\mathrm{Ti}$ & 0.01 & 0.01 & 0.01 & 0.01 & 0.01 & 0.01 & 0.01 & 0.01 & 0.01 \\
\hline $\mathrm{Al}$ & 5.93 & 5.80 & 5.89 & 6.85 & 6.74 & 6.90 & 6.56 & 7.30 & 7.24 \\
\hline $\mathrm{Fe}^{3+}$ & 0.10 & 0.12 & 0.09 & 0.09 & 0.08 & 0.08 & 0.09 & 0.10 & 0.09 \\
\hline $\mathrm{Fe}^{2+}$ & 0.00 & 0.00 & 0.00 & 0.00 & 0.00 & 0.00 & 0.00 & 0.00 & 0.00 \\
\hline $\mathrm{Mg}$ & 0.02 & 0.02 & 0.02 & 0.02 & 0.01 & 0.02 & 0.02 & 0.03 & 0.03 \\
\hline $\mathrm{Ca}$ & 2.10 & $\begin{array}{l}1.90 \\
.20\end{array}$ & 1.98 & 2.85 & 2.87 & 3.09 & 2.82 & 3.44 & 3.39. \\
\hline $\mathrm{Na}$ & 1.90 & 2.08 & 1.99 & 1.11 & 1.17 & 1.01 & 1.34 & 0.60 & 0.66 \\
\hline $\mathrm{K}$ & 0.07 & 0.11 & 0.10 & 0.03 & 0.02 & 0.01 & 0.02 & 0.01 & 0.01 \\
\hline P & 0.01 & 0.01 & 0.01 & 0.02 & 0.02 & 0.01 & 0.01 & 0.02 & 0.02 \\
\hline Sum Z & 5.77 & 15.79 & 15.79 & 15.90 & 15.83 & 15.76 & 15.68 & 15.81 & 15.79 \\
\hline Sum $x$ & 4.07 & 4.09 & 4.07 & 3.99 & 4.07 & 4.11 & 4.18 & 4.05 & 4.06 \\
\hline Orthoclase & 1.77 & 2.61 & 2.46 & 0.76 & 0.58 & 0.23 & 0.51 & 0.24 & 0.23 \\
\hline Albite & 46.59 & 50.93 & 48.85 & 27.72 & 28.75 & 24.63 & 32.02 & 14.79 & 16.33 \\
\hline Anorthite & 51.64 & 46.46 & 48.69 & 71.51 & 70.67 & 75.14 & 67.47 & 84.97 & 83.44 \\
\hline
\end{tabular}


Table 4. Microprobe analyses of alkali feldspar crystals in the tephra layers at Site 758.

\begin{tabular}{|c|c|c|c|c|c|c|c|c|c|c|}
\hline $\begin{array}{l}\text { Sample } \\
\text { Layer }\end{array}$ & $\begin{array}{c}758 \mathrm{C}-1 \mathrm{H}-2 \\
14 \mathrm{~cm} \\
\mathrm{~A}\end{array}$ & $\begin{array}{c}758 \mathrm{~A}-2 \mathrm{H}-4, \\
38 \mathrm{~cm} \\
\text { D }\end{array}$ & $\begin{array}{c}758 \mathrm{~A}-2 \mathrm{H}-4, \\
38 \mathrm{~cm} \\
\text { D }\end{array}$ & $\begin{array}{c}758 \mathrm{~A}-2 \mathrm{H}-4 \\
38 \mathrm{~cm} \\
\mathrm{D}\end{array}$ & $\begin{array}{c}758 \mathrm{~B}-2 \mathrm{H}-2, \\
25 \mathrm{~cm} \\
\mathrm{~d}\end{array}$ & $\begin{array}{c}758 \mathrm{~B}-2 \mathrm{H}-2 \\
25 \mathrm{~cm} \\
\mathrm{~d}\end{array}$ & $\begin{array}{c}758 \mathrm{~B}-2 \mathrm{H}-2 \\
25 \mathrm{~cm} \\
\mathrm{~d}\end{array}$ & $\begin{array}{c}758 \mathrm{~B}-2 \mathrm{H}-2 \\
25 \mathrm{~cm} \\
\mathrm{~d}\end{array}$ & $\begin{array}{c}758 \mathrm{~B}-2 \mathrm{H}-2 \\
25 \mathrm{~cm} \\
\mathrm{~d}\end{array}$ & $\begin{array}{c}758 \mathrm{~B}-2 \mathrm{H}-2, \\
25 \mathrm{~cm} \\
\mathrm{~d}\end{array}$ \\
\hline $\mathrm{SiO}_{2}$ & 65.67 & 65.73 & 66.19 & 65.05 & 66.84 & 66.75 & 65.37 & 65.59 & 66.25 & 65.23 \\
\hline $\mathrm{TiO}_{2}$ & 0.05 & 0.04 & 0.04 & 0.04 & 0.02 & 0.02 & 0.02 & 0.02 & 0.02 & 0.03 \\
\hline $\mathrm{Al}_{2} \mathrm{O}_{3}$ & 19.16 & 18.63 & 18.60 & 18.74 & 18.51 & 18.85 & 18.55 & 18.44 & 18.66 & 18.93 \\
\hline $\mathrm{Fe}_{2} \mathrm{O}_{3}$ & 0.04 & 0.09 & 0.13 & 0.12 & 0.09 & 0.11 & 0.10 & 0.09 & 0.12 & 0.11 \\
\hline $\mathrm{MgO}$ & 0.00 & 0.00 & 0.00 & 0.03 & 0.01 & 0.00 & 0.00 & 0.00 & 0.00 & 0.00 \\
\hline $\mathrm{CaO}$ & 0.07 & 0.18 & 0.19 & 0.19 & 0.21 & 0.19 & 0.17 & 0.15 & 0.17 & 0.20 \\
\hline $\mathrm{K}_{2} \mathrm{O}$ & 14.79 & 13.02 & 13.04 & 12.86 & 12.49 & 12.65 & 12.24 & 12.35 & 12.17 & 12.20 \\
\hline $\mathrm{P}_{2} \mathrm{O}_{5}$ & 0.16 & 0.01 & 0.00 & 0.02 & 0.04 & 0.04 & 0.02 & 0.00 & 0.01 & 0.03 \\
\hline Total & 101.64 & 100.13 & 100.80 & 99.65 & 100.96 & 101.39 & 99.18 & 99.45 & 100.18 & 99.55 \\
\hline $\mathrm{Si}$ & 11.87 & 12.03 & 12.02 & 11.94 & 12.12 & 12.05 & 12.06 & 12.06 & 12.11 & 11.98 \\
\hline $\mathrm{Ti}$ & 0.01 & 0.01 & 0.01 & 0.01 & 0.00 & 0.00 & 0.00 & 0.00 & 0.00 & 0.00 \\
\hline Al & 4.08 & 4.02 & 3.98 & 4.05 & 3.96 & 4.01 & 4.03 & 4.00 & 4.02 & 4.10 \\
\hline $\mathrm{Fe}^{3+}$ & 0.00 & 0.01 & 0.01 & 0.01 & 0.01 & 0.01 & 0.01 & 0.01 & 0.01 & 0.01 \\
\hline $\mathrm{Mg}$ & 0.00 & 0.00 & 0.00 & 0.01 & 0.00 & 0.00 & 0.00 & 0.00 & 0.00 & 0.00 \\
\hline $\mathrm{Na}$ & 0.60 & 0.86 & 0.92 & 0.93 & 0.97 & 0.97 & 0.97 & 1.00 & 0.99 & 1.00 \\
\hline K & 3.41 & 3.04 & 3.02 & 3.01 & 2.89 & 2.91 & 2.88 & 2.90 & 2.84 & 2.86 \\
\hline $\mathrm{P}$ & 0.02 & 0.00 & 0.00 & 0.00 & 0.01 & 0.01 & 0.00 & 0.00 & 0.00 & 0.00 \\
\hline Sum $Z$ & 15.95 & 16.05 & 16.00 & 16.00 & 16.08 & 16.06 & 16.10 & 16.06 & 16.13 & 16.08 \\
\hline Sum $X$ & 4.02 & 3.94 & 3.98 & 3.97 & 3.90 & 3.92 & 3.88 & 3.93 & 3.86 & 3.90 \\
\hline Orthoclase & 84.84 & 77.20 & 75.96 & 75.77 & 74.14 & 74.26 & 74.17 & 73.74 & 73.59 & 73.26 \\
\hline Albite & 14.82 & 21.90 & 23.11 & 23.29 & 24.81 & 24.80 & 24.96 & 25.50 & 25.55 & 25.74 \\
\hline Anorthite & 0.34 & 0.90 & 0.93 & 0.94 & 1.05 & 0.94 & 0.87 & 0.75 & 0.86 & 1.01 \\
\hline
\end{tabular}

Note: The sum of the cations for all analyses is 20 .

Kommission and is part of a Ph.D. dissertation. Farrell's work was supported by a grant from JOI/USSAC (TAMRF-20244).

\section{REFERENCES}

Aldiss, D. T., and Ghazali, S. A., 1984. The regional geology and evolution of the Toba volcano-tectonic depression, Indonesia.J.Geol. Soc. London, 141:487-500.

Bitschene, P. R., and Schmincke, H.-U., 1990. Fallout tephra layers: composition and significance. In Heling, D., Rothe, P., Förstner, U., and Stoffers, P. (Eds.), Sediments and Environmental Geochemistry: Heidelberg (Springer), 48-82.

Bramlette, M. N., and Bradley, W. H., 1942. Geology and biology of North Atlantic deep-sea cores between Newfoundland and Ireland. Pt. I: lithology and geologic interpretations. Geol. Surv. Prof. Pap. U.S., 196A:1-55.

Chesner, C. A., 1988. The Toba tuffs and caldera complex, Sumatra, Indonesia: insights into magma bodies and eruptions [Ph.D. dissert.]. Michigan Tech. Univ,, Houghton.

Deer, W. A., Howie, R. A., and Zussman, J., 1966. An Introduction to the Rock Forming Minerals. Essex, U.K. (Longman Group, Ltd.), 528 p.

Diehl, J. F., Onstott, T. C., Chesner, C. A., and Knight, M. D., 1987. No short reversals of Brunhes age recorded in the Toba Tuffs, North Sumatra, Indonesia. Geophys. Res. Lett., 14:753-766.

Fisher, R. V., 1964. Maximum size, median diameter, and sorting of tephra. J. Geophys. Res., 69:341-355.

Imbrie, J., Hays, J. D., Martinson, D. G., McIntyre, A., Mix, A. C., Morley, J. J., Pisias, N. G., Prell, W. L., and Shackleton, N. J., 1984. The orbital theory of Pleistocene climate: support from a revised chronology of the marine delta $\delta^{18} \mathrm{O}$ record. In Berger, A., Imbrie, J., Hays, J., Kukla, G., and Saltzman, B. (Eds.), Milankovitch and Climate (pt. 1): Dordrecht (D. Reidel), 269-305.

Kennett, J. P., 1981. Marine tephrochronology. In Emiliani, C. (Ed.), The Sea (vol. 7): New York (Wiley), 1373-1436.
Ninkovich, D., 1979. Distribution, age and chemical composition of tephra layers in deep-sea sediments off western Indonesia. J. Volcanol. Geotherm. Res., 5:67-86.

Ninkovich, D., Shackleton, N. J., Abdel-Monem, A. A., Obradovich, J. D., and Izett, G., 1978. K-Ar age of the late Pleistocene eruption of Toba, north Sumatra. Nature, 276:574-577.

Ninkovich, D., Sparks, R.S.J., and Ledbetter, M. T., 1979. The exceptional magnitude and intensity of the Toba eruption, Sumatra: an example of the use of deep-sea tephra layers as a geological tool. Bull. Volcanol., 41:286-297.

Nishimura, S., Abe, E., Yokoyama, T., Wirasantosa, S., and Dharma, A., 1977. Paleolimnol. Lake Biwa Japan Pleistocene, 5:313-332.

Peirce, J., Weissel, J., et al., 1989. Proc. ODP, Init. Repts., 121: College Station, TX (Ocean Drilling Program).

Prell, W. L., Imbrie, J., Martinson, D. G., Morley, J. J., Pisias, N. G., Shackleton, N. J., and Streeter, H. F., 1986. Graphic correlation of oxygen isotope stratigraphy: application to the late Quaternary. $\mathrm{Pa}$ leoceanography, 1:137-162.

Raymo, M. E., Ruddiman, W. F., Backman, J., Clement, B. M., and Martinson, D. G., 1989. Late Pliocene variation in Northern Hemisphere ice sheets and North Atlantic deep water circulation. Paleoceanography, 4:413-446.

Rose, W. I., and Chesner, C. A., 1987. Dispersal of ash in the great Toba eruption, $75 \mathrm{ka}$. Geology, 15:913-917.

1988. World-wide dispersal of ash and gases from Earth's largest known eruption, Toba, Sumatra, $78 \mathrm{ka}$. IGBP (Inter. Alfred Wegner Conf. on the Contribution of Solid Earth Sciences). (Abstract).

Ruddiman, W. F., Cameron, D., and Clement, B. M., 1987. Sediment disturbance and correlation of offset holes drilled with the hydraulic piston corer: Leg 94. In Ruddiman, W. F., Kidd, R. B., Thomas, E., et al., Init. Repts. DSDP, 94, Pt. 2: Washington (U.S. Govt. Printing Office), 615-634.

Ruddiman, W. F., and Glover, L. K., 1982. Mixing of volcanic ash zones in subpolar North Atlantic sediments. In Scrutton, R. A., and Talwani, M. (Eds.), The Ocean Floor: Chicago (Wiley), 37-67. 
Table 4 (continued).

\begin{tabular}{|c|c|c|c|c|c|c|c|c|c|c|c|}
\hline $\begin{array}{l}\text { Sample } \\
\text { Layer }\end{array}$ & $\begin{array}{c}758 \mathrm{~B}-2 \mathrm{H}-2 \\
25 \mathrm{~cm} \\
\mathrm{~d}\end{array}$ & $\begin{array}{c}758 \mathrm{~B}-2 \mathrm{H}-2, \\
25 \mathrm{~cm} \\
\mathrm{~d}\end{array}$ & $\begin{array}{c}758 \mathrm{~B}-3 \mathrm{H}-4 \\
69 \mathrm{~cm} \\
\mathrm{G}\end{array}$ & $\begin{array}{c}758 \mathrm{~B}-3 \mathrm{H}-4 \\
69 \mathrm{~cm} \\
\mathrm{G}\end{array}$ & $\begin{array}{c}758 \mathrm{~B}-6 \mathrm{H}-2 \\
47 \mathrm{~cm} \\
\mathrm{~h}\end{array}$ & $\begin{array}{c}758 \mathrm{~B}-6 \mathrm{H}-2 \\
47 \mathrm{~cm} \\
\mathrm{~h}\end{array}$ & $\begin{array}{c}758 \mathrm{~B}-6 \mathrm{H}-2, \\
47 \mathrm{~cm} \\
\mathrm{~h}\end{array}$ & $\begin{array}{c}758 \mathrm{~B}-6 \mathrm{H}-2 \\
47 \mathrm{~cm} \\
\mathrm{~h}\end{array}$ & $\begin{array}{c}758 \mathrm{~B}-6 \mathrm{H}-7, \\
17 \mathrm{~cm} \\
\mathrm{I}\end{array}$ & $\begin{array}{c}758 \mathrm{~B}-8 \mathrm{H}-4 \\
111 \mathrm{~cm} \\
\mathrm{M}\end{array}$ & $\begin{array}{c}758 \mathrm{~B}-8 \mathrm{H}-4, \\
111 \mathrm{~cm} \\
\mathrm{M}\end{array}$ \\
\hline $\mathrm{SiO}_{2}$ & 66.54 & 66.04 & 65.29 & 65.62 & 66.03 & 65.99 & 65.38 & 65.28 & 64.55 & 64.51 & 63.77 \\
\hline $\mathrm{TiO}_{2}$ & 0.03 & 0.02 & 0.02 & 0.04 & 0.02 & 0.03 & 0.03 & 0.03 & 0.03 & 0.09 & 0.12 \\
\hline $\mathrm{Al}_{2} \mathrm{O}_{3}$ & 18.99 & 18.74 & 18.74 & 18.46 & 18.22 & 18.43 & 18.64 & 18.79 & 18.33 & 18.78 & 18.79 \\
\hline $\mathrm{Fe}_{2} \mathrm{O}_{3}$ & 0.11 & 0.09 & 0.17 & 0.13 & 0.13 & 0.10 & 0.10 & 0.07 & 0.02 & 0.44 & 0.51 \\
\hline $\mathrm{MgO}$ & 0.00 & 0.00 & 0.00 & 0.00 & 0.00 & 0.00 & 0.00 & 0.00 & 0.03 & 0.00 & 0.01 \\
\hline $\mathrm{CaO}$ & 0.19 & 0.16 & 0.27 & 0.25 & 0.16 & 0.14 & 0.17 & 0.15 & 0.08 & 1.08 & 1.24 \\
\hline $\mathrm{Na}_{2} \mathrm{O}$ & 2.77 & 2.66 & 3.19 & 3.22 & 2.77 & 2.79 & 2.85 & 2.84 & 0.81 & 6.35 & 6.82 \\
\hline $\mathrm{K}_{2} \mathrm{O}$ & 12.59 & 12.70 & 12.09 & 11.95 & 12.96 & 12.87 & 12.56 & 12.81 & 16.24 & 7.06 & 5.96 \\
\hline $\mathrm{P}_{2} \mathrm{O}_{5}$ & 0.02 & 0.02 & 0.02 & 0.01 & 0.02 & 0.04 & 0.04 & 0.05 & 0.17 & 0.04 & 0.04 \\
\hline Total & 101.24 & 100.43 & 99.79 & 99.68 & 100.31 & 100.39 & 99.77 & 100.02 & 100.26 & 98.35 & 97.26 \\
\hline $\mathrm{Si}$ & 12.03 & 12.04 & 11.94 & 12.01 & 12.04 & 12.02 & 11.97 & 11.92 & 11.87 & 11.78 & 11.75 \\
\hline $\mathrm{Ti}$ & 0.00 & 0.00 & 0.00 & 0.01 & 0.00 & 0.00 & 0.00 & 0.00 & 0.00 & 0.01 & 0.02 \\
\hline $\mathrm{Al}$ & 4.04 & 4.03 & 4.04 & 3.98 & 3.92 & 3.96 & 4.02 & 4.04 & 3.97 & 4.04 & 4.08 \\
\hline $\mathrm{Fe}^{3+}$ & 0.01 & 0.01 & 0.02 & 0.01 & 0.01 & 0.01 & 0.01 & 0.01 & 0.00 & 0.05 & 0.06 \\
\hline $\mathrm{Mg}$ & 0.00 & 0.00 & 0.00 & 0.00 & 0.00 & 0.00 & 0.00 & 0.00 & 0.01 & 0.00 & 0.00 \\
\hline $\mathrm{Ca}$ & 0.04 & 0.03 & 0.05 & 0.05 & 0.03 & 0.03 & 0.03 & 0.03 & 0.02 & 0.21 & 0.24 \\
\hline $\mathrm{Na}$ & 0.97 & 0.94 & 1.13 & 1.14 & 0.98 & 0.99 & 1.01 & 1.01 & 0.29 & 2.25 & 2.44 \\
\hline K & 2.90 & 2.95 & 2.82 & 2.79 & 3.01 & 2.99 & 2.93 & 2.98 & 3.81 & 1.65 & 1.40 \\
\hline P & 0.00 & 0.00 & 0.00 & 0.00 & 0.00 & 0.01 & 0.01 & 0.01 & 0.03 & 0.01 & 0.01 \\
\hline Sum Z & 16.07 & 16.06 & 15.97 & 16.00 & 15.95 & 15.98 & 16.00 & 15.96 & 15.84 & 15.83 & 15.83 \\
\hline Sum X & 3.91 & 3.92 & 4.00 & 3.98 & 4.02 & 4.00 & 3.98 & 4.02 & 4.11 & 4.11 & 4.08 \\
\hline Orthoclase & 74.23 & 75.25 & 70.43 & 70.07 & 74.89 & 74.70 & 73.73 & 74.25 & 92.60 & 40.07 & 34.32 \\
\hline Albite & 24.82 & 23.96 & 28.25 & 28.70 & 24.33 & 24.61 & 25.43 & 25.02 & 7.02 & 54.78 & 59.69 \\
\hline Anorthite & 0.94 & 0.80 & 1.32 & 1.23 & 0.78 & 0.68 & 0.84 & 0.73 & 0.38 & 5.15 & 6.00 \\
\hline
\end{tabular}

Ruddiman, W. F., Raymo, M. E., Martinson, D. G., Clement, B. M., and Backman, J., 1989. Pleistocene evolution: Northern Hemisphere ice sheets and North Atlantic Ocean. Paleoceanography, 4:353-412.

Schmincke, H.-U., 1981. Ash from vitric muds in deep sea cores from the Mariana Trough and fore-arc regions (south Philippine Sea) (Sites $453,454,455,458,459)$. In Hussong, D. M., Uyeda, S., et al., Init. Repts. DSDP, 60: Washington (U.S. Govt. Printing Office), 473-481.

Shackleton, N. J., and Hall, M. A., 1989. Stable isotope history of the Pleistocene at ODP Site 677. In Becker, K., Sakai, H., et al., Proc. ODP, Sci. Results, 111: College Station, TX (Ocean Drilling Program), 295-316.

Shaw, D. M., Watkins, N. D., and Huang, T. C., 1974. Atmospherically transported volcanic glass in deep sea sediments: theoretical considerations. J. Geophys. Res., 79:3087-3094.

Shipboard Scientific Party, 1989. Site 758. In Peirce, J., Weissel, J., et al., Proc. ODP, Init. Repts., 121: College Station, TX (Ocean Drilling Program), 359-453.

Smith, R. L., and Bailey, R. A., 1968. Resurgent cauldrons. In Coats, R. R., Hay, R. L., and Anderson, C. A. (Eds.), Studies in Volcanology: Geol. Soc. Am. Mem., 116:613-662.

von der Borch, C. C., Sclater, J. G., et al., 1974. Init. Repts. DSDP, 22: Washington (U.S. Govt. Printing Office).

Walker, G.P.L., 1971. Grain-size characteristics of pyroclastic deposits. J. Geol., 79:696-714.

Williams, M.A.J., and Royce, K., 1982. Quaternary geology of the middle Son Valley, north-central India: implications for prehistoric archaeology. Paleogeogr., Paleoclimatol., Paleoecol., 38:139-162.

Date of initial receipt: 13 March 1990

Date of acceptance: 31 October 1990

Ms 121B-123

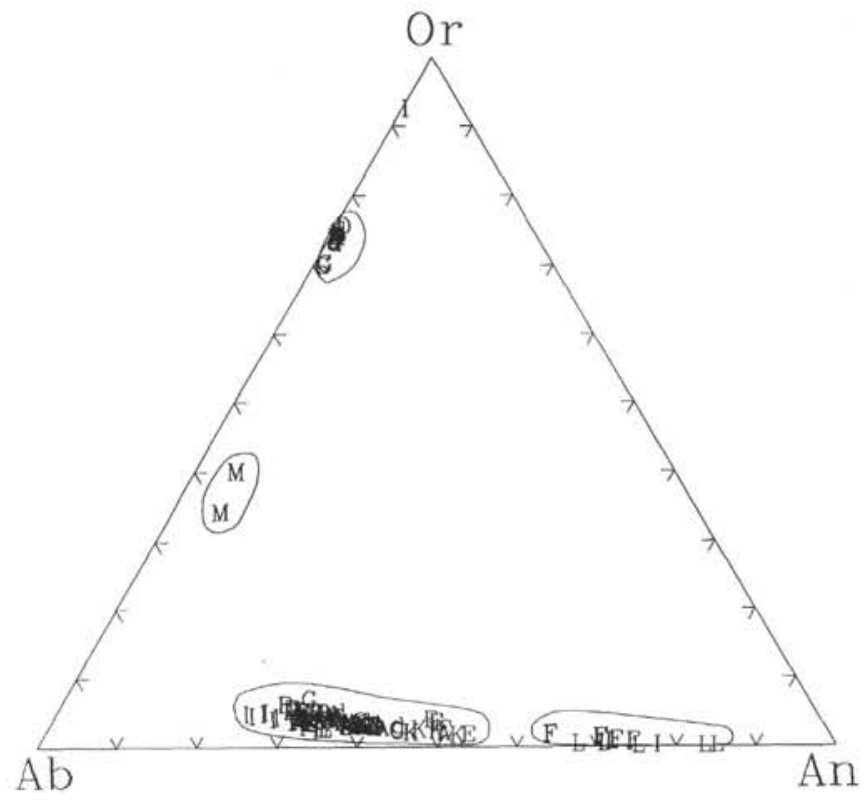

Figure 7. Ternary diagram of feldspars in the Site 758 tephras. The analyses fall into four distinct groups of An65-85, An25-55, Or40, and Or70-76. Layers I, G, D, and d have sanidine, as well as andesine, crystals. Layers F, I, and L contain bytownite crystals, which is unusually rich in An for rhyolitic magmas. 
Table 5. Microprobe analyses of major oxide concentrations in other crystals in the tephra layers at Site 758.

\begin{tabular}{|c|c|c|c|c|c|c|c|c|c|c|c|c|c|c|}
\hline $\begin{array}{l}\text { Sample } \\
\text { Layer }\end{array}$ & $\begin{array}{c}758 \mathrm{~B}-1 \mathrm{H}-1 . \\
114 \mathrm{~cm} \\
\mathrm{~A}\end{array}$ & $\begin{array}{c}758 \mathrm{C}-1 \mathrm{H}-2 \\
14 \mathrm{~cm} \\
\mathrm{~A}\end{array}$ & $\begin{array}{c}758 \mathrm{C}-1 \mathrm{H}-2 \\
27 \mathrm{~cm} \\
\mathrm{~A}\end{array}$ & $\begin{array}{c}758 \mathrm{C}-1 \mathrm{H}-2 \\
27 \mathrm{~cm} \\
\mathrm{~A}\end{array}$ & $\begin{array}{c}758 \mathrm{C}-1 \mathrm{H}-2 \\
27 \mathrm{~cm} \\
\mathrm{~A}\end{array}$ & $\begin{array}{c}758 \mathrm{~A}-1 \mathrm{H}-2 \\
47 \mathrm{~cm} \\
\mathrm{~B}\end{array}$ & $\begin{array}{c}758 \mathrm{~A}-1 \mathrm{H}-2 \\
47 \mathrm{~cm} \\
\mathrm{~B}\end{array}$ & $\begin{array}{c}758 \mathrm{~A}-1 \mathrm{H}-2 \\
47 \mathrm{~cm} \\
\mathrm{~B}\end{array}$ & $\begin{array}{c}758 \mathrm{~A}-1 \mathrm{H}-2 \\
47 \mathrm{~cm} \\
\mathrm{~B}\end{array}$ & $\begin{array}{c}758 \mathrm{~A}-1 \mathrm{H}-2 \\
47 \mathrm{~cm} \\
\mathrm{~B}\end{array}$ & $\begin{array}{c}758 \mathrm{~A}-1 \mathrm{H}-2 \\
47 \mathrm{~cm} \\
\mathrm{~B}\end{array}$ & $\begin{array}{c}758 \mathrm{~A}-1 \mathrm{H}-2 \\
47 \mathrm{~cm} \\
\mathrm{~B}\end{array}$ & $\begin{array}{c}758 \mathrm{~A}-4 \mathrm{H}-4 \\
3 \mathrm{~cm} \\
\mathrm{H}\end{array}$ & $\begin{array}{c}758 \mathrm{~A}-7 \mathrm{H}-2 \\
120 \mathrm{~cm} \\
1\end{array}$ \\
\hline $\mathrm{SiO}_{2}$ & 45.34 & 0.03 & 36.39 & 34.23 & 35.11 & 36.72 & 36.58 & 35.80 & 36.09 & 37.07 & 37.02 & 36.15 & 0.27 & 34.99 \\
\hline $\mathrm{Al}_{2} \mathrm{O}_{3}$ & 8.61 & 0.00 & 14.16 & 15.23 & 14.35 & 13.86 & 14.23 & 14.03 & 14.40 & 14.27 & 13.97 & 14.26 & 0.00 & 15.14 \\
\hline $\mathrm{FeO}$ & 21.57 & 0.05 & 21.02 & 24.28 & 22.20 & 24.24 & 23.62 & 23.17 & 21.55 & 21.45 & 21.94 & 22.84 & 0.46 & 21.79 \\
\hline $\mathrm{MgO}$ & 8.35 & 0.02 & 9.93 & 7.70 & 9.42 & 8.82 & 9.13 & 9.01 & 9.50 & 9.94 & 9.22 & 9.23 & 0.30 & 10.00 \\
\hline $\mathrm{CaO}$ & 10.34 & 52.50 & 0.05 & 0.04 & 0.05 & 0.01 & 0.01 & 0.03 & 0.02 & 0.01 & 0.00 & 0.01 & 51.54 & 0.04 \\
\hline $\mathrm{K}_{2} \mathrm{O}$ & 1.04 & 0.02 & 8.48 & 8.48 & 8.31 & 9.20 & 9.27 & 9.05 & 8.87 & 9.29 & 9.39 & 9.28 & 0.03 & 8.66 \\
\hline $\mathrm{Na}_{2} \mathrm{O}$ & 1.52 & 0.07 & 0.48 & 0.81 & 0.44 & 0.40 & 0.34 & 0.35 & 0.44 & 0.40 & 0.35 & 0.35 & 0.04 & 0.38 \\
\hline $\mathrm{TiO}_{2}$ & 1.70 & 0.06 & 4.31 & 3.71 & 4.02 & 3.73 & 4.06 & 3.85 & 4.66 & 4.49 & 4.12 & 4.15 & 0.04 & 3.19 \\
\hline $\mathrm{P}_{2} \mathrm{O}_{5}^{-}$ & 0.17 & 42.33 & 0.02 & 0.05 & 0.05 & 0.04 & 0.02 & 0.07 & 0.01 & 0.01 & 0.02 & 0.05 & 41.58 & 0.04 \\
\hline Total & 98.64 & 95.08 & 94.84 & 94.53 & 93.95 & 97.02 & 97.26 & 95.36 & 95.54 & 96.93 & 96.03 & 96.32 & 94.26 & 94.23 \\
\hline Mineral & Amphibole & Apatite & Biotite & Biotite & Biotite & Biotite & Biotite & Biotite & Biotite & Biotite & Biotite & Biotite & Apatite & Biotite \\
\hline
\end{tabular}

\begin{tabular}{|c|c|c|c|c|c|c|c|c|c|c|c|c|c|c|}
\hline $\begin{array}{c}758 \mathrm{~A}-7 \mathrm{H}-2 \\
120 \mathrm{~cm} \\
1\end{array}$ & $\begin{array}{c}758 \mathrm{~B}-1 \mathrm{H}-5 \\
119 \mathrm{~cm} \\
\mathrm{C}\end{array}$ & $\begin{array}{c}758 \mathrm{~B}-2 \mathrm{H}-2, \\
25 \mathrm{~cm} \\
\mathrm{~d}\end{array}$ & $\begin{array}{c}758 \mathrm{~B}-2 \mathrm{H}-2, \\
25 \mathrm{~cm} \\
\mathrm{~d}\end{array}$ & $\begin{array}{c}758 \mathrm{~B}-2 \mathrm{H}-2 \\
25 \mathrm{~cm} \\
\mathrm{~d}\end{array}$ & $\begin{array}{c}758 \mathrm{~B}-2 \mathrm{H}-2 \\
25 \mathrm{~cm} \\
\mathrm{~d}\end{array}$ & $\begin{array}{c}758 \mathrm{~B} \cdot 2 \mathrm{H}-2 . \\
25 \mathrm{~cm} \\
\mathrm{~d}\end{array}$ & $\begin{array}{c}758 \mathrm{~B}-2 \mathrm{H}-2 \\
25 \mathrm{~cm} \\
\mathrm{~d}\end{array}$ & $\begin{array}{c}758 \mathrm{~B}-3 \mathrm{H}-4 \\
69 \mathrm{~cm} \\
\mathrm{G}\end{array}$ & $\begin{array}{c}758 \mathrm{~B}-4 \mathrm{H}-3 \\
8 \mathrm{~cm} \\
\mathrm{H}\end{array}$ & $\begin{array}{c}758 \mathrm{~B}-6 \mathrm{H}-2, \\
47 \mathrm{~cm} \\
\mathrm{~h}\end{array}$ & $\begin{array}{c}758 \mathrm{~B}-6 \mathrm{H}-2 \\
47 \mathrm{~cm} \\
\mathrm{~h}\end{array}$ & $\begin{array}{c}758 \mathrm{~B}-6 \mathrm{H}-2 \\
47 \mathrm{~cm} \\
\mathrm{~h}\end{array}$ & $\begin{array}{c}758 \mathrm{~B}-6 \mathrm{H}-7, \\
17 \mathrm{~cm} \\
\mathrm{I}\end{array}$ & $\begin{array}{c}758 \mathrm{~B}-7 \mathrm{H}-6, \\
29 \mathrm{~cm} \\
\mathrm{~K}\end{array}$ \\
\hline 36.32 & 34.97 & 0.68 & 34.78 & 34.21 & 35.66 & 36.18 & 1.01 & 35.80 & 0.18 & 35.15 & 31.52 & 36.12 & 35.80 & 50.66 \\
\hline 15.11 & 14.31 & 0.00 & 13.92 & 14.42 & 13.74 & 13.79 & 0.20 & 14.31 & 0.00 & 14.34 & 5.24 & 14.17 & 14.53 & 1.49 \\
\hline 19.92 & 23.97 & 0.63 & 25.35 & 25.47 & 25.52 & 25.40 & 1.37 & 15.43 & 1.32 & 24.67 & 0.62 & 24.45 & 21.89 & 9.52 \\
\hline 11.21 & 7.71 & 0.01 & 8.21 & 8.25 & 8.38 & 8.20 & 0.15 & 13.36 & 0.15 & 7.92 & 0.01 & 7.85 & 9.81 & 13.86 \\
\hline 0.04 & 0.03 & 52.46 & 0.02 & 0.03 & 0.04 & 0.02 & 52.11 & 0.04 & 53.22 & 0.05 & 32.56 & 0.07 & 0.06 & 20.53 \\
\hline 8.39 & 8.20 & 0.14 & 8.38 & 8.44 & 8.63 & 8.77 & 0.40 & 8.81 & 0.00 & 8.74 & 1.79 & 8.73 & 8.99 & 0.04 \\
\hline 0.52 & 0.40 & 0.08 & 0.38 & 0.36 & 0.54 & 0.37 & 0.11 & 0.45 & 0.10 & 0.33 & 0.76 & 0.44 & 0.35 & 0.26 \\
\hline 3.85 & 4.77 & 0.07 & 3.66 & 3.59 & 3.76 & 3.63 & 0.15 & 4.65 & 0.06 & 3.71 & 0.05 & 3.62 & 3.12 & 0.39 \\
\hline 0.03 & 0.04 & 41.21 & 0.07 & 0.06 & 0.03 & 0.06 & 40.93 & 0.04 & 41.21 & 0.05 & 26.65 & 0.03 & 0.06 & 0.10 \\
\hline 95.39 & 94.40 & 95.28 & 94.77 & 94.83 & 96.30 & 96.42 & 96.43 & 92.89 & 96.24 & 94.96 & 99.20 & 95.48 & 94.61 & 96.85 \\
\hline Biotite & Biotite & Apatite & Biotite & Biotite & Biotite & Biotite & Apatite & Biotite & Apatite & Biotite & Biotite & Biotite & Biotite & Amphibole \\
\hline
\end{tabular}




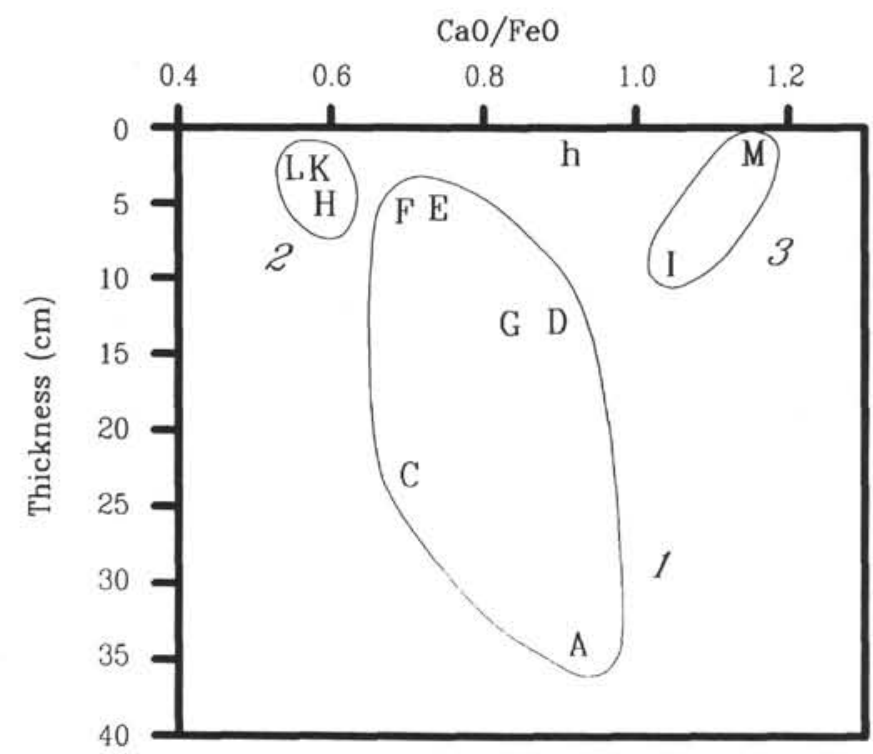

Figure 8. Plot of $\mathrm{CaO} / \mathrm{FeO}$ ratio vs. thickness of the tephra layers. The three cogenetic groups display increasing tephra layer thickness with time and evolution of the magmas.

Table 6. Summary of the age of major tephra layers.

\begin{tabular}{|c|c|c|c|c|c|c|}
\hline \multirow[b]{2}{*}{ Layer } & \multicolumn{2}{|c|}{ Age (Ma) } & \multirow{2}{*}{$\begin{array}{c}\text { Interval } \\
\text { between } \\
\text { layers (Ma) }\end{array}$} & \multicolumn{3}{|c|}{ Group $^{a}$} \\
\hline & paleomagnetic & ${ }^{18} \mathrm{O}$ & & 1 & 2 & 3 \\
\hline A & $0.092-0.112$ & $0.071-0.080$ & & * & & \\
\hline C & $0.512-0.515$ & $0.513-0.538$ & $0.400-0.467$ & * & & \\
\hline D & $0.734-0.741$ & $0.731-0.750$ & $0.193-0.238$ & * & & \\
\hline E & $0.776-0.779$ & 0.775 & $0.029-0.040$ & $*$ & & \\
\hline F & $1.277-1.284$ & $1.273-1.294$ & $0.302-0.381$ & $*$ & & \\
\hline G & $1.645-1.654$ & $1.596-1.636$ & $0.584-0.669$ & $* ?$ & $\bullet ?$ & \\
\hline $\mathrm{H}$ & $2.261-2.265$ & $2.238-2.260$ & $1.399-1.428$ & & * & \\
\hline h & $3.664-3.666$ & & $0.458-0.466$ & & & *? \\
\hline 1 & $4.124-4.130$ & & $0.422-0.435$ & & & * \\
\hline J & $4.552-4.559$ & & $0.107-0.115$ & & * & \\
\hline K & $4.666-4.667$ & & $0.092-0.096$ & & * & \\
\hline L. & $4.759-4.762$ & & $0.300-0.304$ & & - & \\
\hline M & $5.062-5.063$ & & & & & - \\
\hline
\end{tabular}

${ }^{a}$ Unclear group identification is noted with a question mark. 